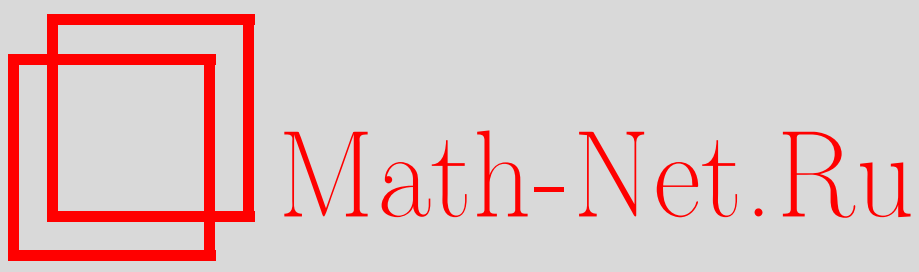

Е. Ю. Панов, К теории обобщенных энтропийных решений задачи Коши для квазилинейного уравнения первого порядка в классе локально суммируемых функций, Изв. РАН. Сер. матем., 2002, том 66, выпуск 6, 91-136

DOI: https://doi.org/10.4213/im411

Использование Общероссийского математического портала Math-Net.Ru подразумевает, что вы прочитали и согласны с пользовательским соглашением

http://www . mathnet.ru/rus/agreement

Параметры загрузки:

IP : 54.198 .67 .100

26 апреля 2023 г., 14:25:47

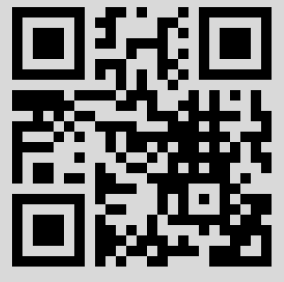


УДК 517.95

\author{
Е. Ю. Панов
}

\title{
К теории обобщенных энтропийных решений задачи Коши для квазилинейного уравнения первого порядка в классе локально суммируемых функций
}

\begin{abstract}
Построена теория локально суммируемых обобщенных энтропийных решений (о.э.р.) задачи Коши для неоднородного квазилинейного уравнения первого порядка с лишь непрерывным вектором потока, удовлетворяющим линейному ограничению на рост. Доказано существование наибольшего и наименьшего о.э.p., приведены достаточные условия единственности о.э.р. Доказаны различные варианты принципа сравнения, даны оценки $L^{p}$-норм решения по пространственным переменным. Установлена единственность о.э.р. в случае периодических по пространственным переменным входных данных.

Библиограффия: 20 наименований.
\end{abstract}

\section{§ 1. Введение}

Рассмотрим задачу Коши для квазилинейного уравнения первого порядка

$$
u_{t}+\operatorname{div}_{x} \varphi(u)=g(t, x, u),
$$

где $\varphi=\left(\varphi_{1}, \ldots, \varphi_{n}\right), u=u(t, x),(t, x) \in \Pi_{T}=(0, T) \times \mathbb{R}^{n}, T>0$, с начальным условием

$$
u(0, x)=u_{0}(x) \in L_{\mathrm{loc}}^{1}\left(\mathbb{R}^{n}\right) .
$$

Предполагается, что вектор потока $\varphi(u)$ лишь непрерывен: $\varphi(u) \in C\left(\mathbb{R}, \mathbb{R}^{n}\right)$, и удовлетворяет следуюшему ограничению на рост:

$$
|\varphi(u)| \leqslant C_{0}(1+|u|), \quad C_{0}=\text { const }
$$

здесь и ниже $|\varphi|$ означает евклидову норму вектора $\varphi \in \mathbb{R}^{n}$.

Обозначим $\bar{\Pi}_{T}=[0, T) \times \mathbb{R}^{n}$ и предположим, что функция источника $g=$ $g(t, x, u) \in L_{\text {lос }}^{1}\left(\bar{\Pi}_{T}\right)$, при любом фиксированном $u \in \mathbb{R}$, непрерывна по переменной $u$ для всех $(t, x) \in \Pi_{T}$ и при некоторой константе $L \geqslant 0$

$$
g(t, x, u)-g(t, x, v) \leqslant L(u-v) \quad \forall u, v \in \mathbb{R}, \quad u>v .
$$

Из приведенных выше условий, в частности, следует, что для любой функции $u(t, x) \in L_{\text {loc }}^{1}\left(\Pi_{T}\right)$ композищия $g(t, x, u(t, x))$ измерима на $\Pi_{T}$.

Выполнено при поддержке Российского фонда фундаментальных исследований (грант РФФИ № 00-01-00921 и грант Президента РФ № 00-15-99254). 
ОПРЕДЕЛЕНИЕ 1 [10], [11]. Локально суммируемая функция $u=u(t, x) \in$ $L_{\text {lос }}^{1}\left(\Pi_{T}\right)$ называется обобщенным әнтропийным решением (коротко, о.э.р.) задачи Коши (1), (2), если $g=g(t, x, u(t, x)) \in L_{\mathrm{loc}}^{1}\left(\Pi_{T}\right)$ и

a) $\forall k \in \mathbb{R}$

$$
|u-k|_{t}+\operatorname{div}_{x}[\operatorname{sign}(u-k)(\varphi(u)-\varphi(k))] \leqslant \operatorname{sign}(u-k) g
$$

в смысле распределений на $\Pi_{T}\left(\right.$ в $\left.\mathscr{D}^{\prime}\left(\Pi_{T}\right)\right)$;

b) ess $\lim _{t \rightarrow 0+} u(t, \cdot)=u_{0}$ в $L_{\text {loc }}^{1}\left(\mathbb{R}^{n}\right)$, т.е. сушествует множество $\mathscr{E} \subset(0, T)$ полной меры Лебега такое, что $u(t, \cdot) \in L_{\text {loc }}^{1}\left(\mathbb{R}^{n}\right), t \in \mathscr{E}$, и $u(t, \cdot) \rightarrow u_{0}$ в $L_{\text {loc }}^{1}\left(\mathbb{R}^{n}\right)$ при $t \rightarrow 0+, t \in \mathscr{E}$.

Условие (5) означает, что для любой пробной функции $f=f(t, x) \in C_{0}^{\infty}\left(\Pi_{T}\right)$, $f \geqslant 0$, вьполнено

$$
\int_{\Pi_{T}}\left\{|u-k| f_{t}+\operatorname{sign}(u-k)\left[\left(\varphi(u)-\varphi(k), \nabla_{x} f\right)+g f\right]\right\} d t d x \geqslant 0
$$

здесь и ниже $(\cdot, \cdot)$ означает скалярное умножение на $\mathbb{R}^{n}$.

Заметим, что из условия (3) вытекает, что распределение в левой части условия (5) корректно определено.

Хорошо известно (см., например, [4], [11]), что условие (5) можно записать в эквивалентной форме: для любой выпуклой функции $\eta(u) \in C^{1}(\mathbb{R})$ (энтропии), удовлетворяющей ограничению $\left|\eta^{\prime}(u)\right| \leqslant$ const, имеем

$$
\eta(u)_{t}+\operatorname{div}_{x} \psi(u) \leqslant \eta^{\prime}(u) g \quad \text { в } \mathscr{D}^{\prime}\left(\Pi_{T}\right),
$$

где $\psi(u)=\left(\psi_{1}(u), \ldots, \psi_{n}(u)\right)$ - соответствующий вектор потока энтропии, определяемый равенством

$$
\psi(u)=\eta^{\prime}(u) \varphi(u)-\int_{0}^{u} \varphi(\sigma) d \eta^{\prime}(\sigma)
$$

(так что в случае гладкого потока $\varphi(u) \in C^{1}\left(\mathbb{R}, \mathbb{R}^{n}\right)$ вектор $\psi(u)$ определен с точностью до аддитивной константы равенством $\left.\psi^{\prime}(u)=\eta^{\prime}(u) \varphi^{\prime}(u)\right)$.

Заметим, что из условия $\left|\eta^{\prime}(u)\right| \leqslant$ const вытекает, что энтропия $\eta(u)$ и соответствующий вектор потока $\psi(u)$ удовлетворяют линейному ограничению на рост, откуда следует, что в $(6) \eta(u(t, x)) \in L_{\text {loc }}^{1}\left(\Pi_{T}\right), \psi_{i}(u(t, x)) \in L_{\text {loc }}^{1}\left(\Pi_{T}\right), i=1, \ldots, n$, и распределение в левой части неравенства (6) определено.

Можно ввести о.э.р. $u \in L_{\text {loc }}^{p}$ для $p>1$, заменив $(3)$ на требование $|\varphi(u)| \leqslant$ $C_{0}\left(1+|u|^{p}\right)$ (без каких-либо ограничений при $\left.p=\infty\right)$. Однако в этом случае задача Коши оказывается некорректной. Даже при $n=1, g \equiv 0$ ни один из положительных результатов, приведенных ниже, в общем случае не верен. Так, например, в [9] построены ненулевые локально ограниченные о.э.р. задачи Коши для уравнения $u_{t}+\left(u^{3}\right)_{x}=0$ с нулевыми начальными данными. Этот пример легко обобщается на случай функции потока $\varphi(u)=|u|^{p-1} u, p>1$.

Обозначим $C_{0}^{\infty}\left(\bar{\Pi}_{T}\right)$ подпространство функций из $C^{\infty}\left(\bar{\Pi}_{T}\right)$ с компактным носителем (которьй, таким образом, может иметь непустое пересечение с начальной гиперплоскостью $\{t=0\})$. Пусть $f^{+}=\max (f, 0), f^{-}=\max (-f, 0)$. Введем обозначение $\chi_{u>v}$ для характеристической функции множества независимых переменных, на котором выполнено сравнение $u>v$ для двух данных функций $u$ и $v$. Аналогично определяются функции $\chi_{u<v}, \chi_{u=v}$ и т.д. Пусть $\operatorname{sign}^{+}(r)=\chi_{r>0}-$ функция Хевисайда, $\operatorname{sign}^{-}(r)=-\operatorname{sign}^{+}(-r)$.

Дадим следующую полезную переформулировку определения 1. 
ПРЕДЛОЖЕНИЕ 1. Функиия $u=u(t, x) \in L_{\mathrm{loc}}^{1}\left(\bar{\Pi}_{T}\right)$ является о.э.p. задачи (1), (2) тогда и только тогда, когда для любого $k \in \mathbb{R}$ и любой пробной функиии $f=f(t, x) \in C_{0}^{\infty}\left(\bar{\Pi}_{T}\right), f \geqslant 0$, имеем

$$
\begin{gathered}
\int_{\Pi_{T}}\left\{|u-k| f_{t}+\operatorname{sign}(u-k)\left[\left(\varphi(u)-\varphi(k), \nabla_{x} f\right)+g f\right]\right\} d t d x \\
+\int_{\mathbb{R}^{n}}\left|u_{0}(x)-k\right| f(0, x) d x \geqslant 0 .
\end{gathered}
$$

ДокАЗАТЕЛЬСТво. Пусть $u=u(t, x)$ - о.э.р. задачи $(1),(2) ; k \in \mathbb{R}, f=$ $f(t, x) \in C_{0}^{\infty}\left(\bar{\Pi}_{T}\right), f \geqslant 0$. Заметим, прежде всего, что ввиду условия (4)

$$
\begin{aligned}
\operatorname{sign}(u-k) g & \leqslant|g(t, x, k)|+\operatorname{sign}(u-k)(g(t, x, u(t, x))-g(t, x, k)) \\
& \leqslant|g(t, x, k)|+L|u(t, x)-k| \in L_{\mathrm{loc}}^{1}\left(\bar{\Pi}_{T}\right)
\end{aligned}
$$

и $(\operatorname{sign}(u-k) g)^{+} \in L_{\mathrm{loc}}^{1}\left(\bar{\Pi}_{T}\right)$. Поэтому интеграл $\int_{\Pi_{T}} \operatorname{sign}(u-k) g f d t d x \geqslant-\infty$ определен и неравенство (8) имеет смысл.

Выберем функцию $\beta(s) \in C_{0}^{\infty}(\mathbb{R}), \operatorname{supp} \beta(s) \subset[0,1], \beta(s) \geqslant 0, \int \beta(s) d s=1$, и положим $\delta_{\nu}(s)=\nu \beta(\nu s), \theta_{\nu}(t)=\int_{-\infty}^{t} \delta_{\nu}(s) d s$ при $\nu \in \mathbb{N}$. Понятно, что последовательность $\delta_{\nu}(s)$ сходится к $\delta$-мере Дирака в $\mathscr{D}^{\prime}(\mathbb{R})$ при $\nu \rightarrow \infty ; 0 \leqslant \theta_{\nu}(t) \leqslant 1$, $\theta_{\nu}(t)=0$ при $t \leqslant 0, \theta_{\nu}(t)=1$ при $t \geqslant 1 / \nu$ и, следовательно, последовательность $\theta_{\nu}(t)$ сходится поточечно при $\nu \rightarrow \infty$ к функции Хевисайда $\operatorname{sign}^{+}(t)$.

Применяя энтропийное неравенство (5) к пробной функции $f_{\nu}=f_{\nu}(t, x)=$ $f(t, x) \theta_{\nu}\left(t-t_{0}\right) \in C_{0}^{\infty}\left(\Pi_{T}\right), t_{0} \in(0, T)$, получим, что

$$
\begin{aligned}
& \int_{0}^{T} \delta_{\nu}\left(t-t_{0}\right) \int_{\mathbb{R}^{n}}|u(t, x)-k| f(t, x) d x d t \\
& +\int_{\Pi_{T}}\left\{|u-k| f_{t}+\operatorname{sign}(u-k)\left[\left(\varphi(u)-\varphi(k), \nabla_{x} f\right)+g f\right]\right\} \theta_{\nu}\left(t-t_{0}\right) d t d x \geqslant 0 .
\end{aligned}
$$

Пусть $\mathscr{E} 1$ - множество общих точек Лебега для функций

$$
t \mapsto F(k, t)=\int_{\mathbb{R}^{n}}|u(t, x)-k| f(t, x) d x,
$$

где $k \in \mathbb{Q}$ рационально. Ввиду счетности $\mathbb{Q}$ множество $\mathscr{E} 1$ имеет полную меру Лебега на $(0, T)$. Очевидно, отображение $k \mapsto F(k, t)-F(0, t) \in L^{\infty}([0, T])$ непрерывно, равномерно по $t \in[0, T]$, и так как $\mathbb{Q}$ всюду плотно, мы заключаем, что точки $t \in \mathscr{E}_{1}$ - точки Лебега функций $F(k, t)$ для всех $k \in \mathbb{R}$. Пусть $\mathscr{E}_{2} \subset(0, T)-$ множество полной меры из определения 1 такое, что $u(t, \cdot) \in L_{\mathrm{loc}}^{1}\left(\mathbb{R}^{n}\right), t \in \mathscr{E}_{2}$, и $u(t, \cdot) \rightarrow u_{0}$ в $L_{\text {loc }}^{1}\left(\mathbb{R}^{n}\right)$ при $t \rightarrow 0+, t \in \mathscr{E}_{2}$. Положим $\mathscr{E}=\mathscr{E}_{1} \cap \mathscr{E}_{2}$. Ясно, что $\mathscr{E}$ является множеством полной меры на $(0, T)$, и если $t_{0} \in \mathscr{E}$, то в пределе при $\nu \rightarrow \infty$ соотношение (9) преобразуется к неравенству

$$
\begin{aligned}
& \int_{\mathbb{R}^{n}}\left|u\left(t_{0}, x\right)-k\right| f\left(t_{0}, x\right) d x d t \\
& \quad+\int_{\Pi_{T}}\left\{|u-k| f_{t}+\operatorname{sign}(u-k)\left[\left(\varphi(u)-\varphi(k), \nabla_{x} f\right)+g f\right]\right\} \operatorname{sign}^{+}\left(t-t_{0}\right) d t d x \geqslant 0,
\end{aligned}
$$


откуда, после перехода к пределу при $t_{0} \rightarrow 0, t_{0} \in \mathscr{E}($ с учетом требования b) определения 1) вытекает (8).

Для доказательства обратной импликации предположим, что функция $u=$ $u(t, x) \in L_{\text {loc }}^{1}\left(\bar{\Pi}_{T}\right)$ удовлетворяет условию (8). Как было уже отмечено выше, $(\operatorname{sign}(u-k) g)^{+} \in L_{\mathrm{loc}}^{1}\left(\bar{\Pi}_{T}\right)$. Переписывая (8) в форме

$$
\begin{aligned}
\int_{\Pi_{T}}\left\{|u-k| f_{t}+\operatorname{sign}(u-k)\left(\varphi(u)-\varphi(k), \nabla_{x} f\right)\right. \\
\left.\quad+(\operatorname{sign}(u-k) g)^{+} f\right\} d t d x+\int_{\mathbb{R}^{n}}\left|u_{0}(x)-k\right| f(0, x) d x \\
\geqslant \int_{\Pi_{T}}(\operatorname{sign}(u-k) g)^{-} f d t d x
\end{aligned}
$$

и учитывая произвольность $f \in C_{0}^{\infty}\left(\bar{\Pi}_{T}\right), f \geqslant 0$, заключаем: $(\operatorname{sign}(u-k) g)^{-} \in$ $L_{\text {loc }}^{1}\left(\bar{\Pi}_{T}\right)$. Итак, $\operatorname{sign}(u-k) g \in L_{\text {loc }}^{1}\left(\bar{\Pi}_{T}\right)$ и, ввиду произвольности $k \in \mathbb{R}, g=$ $g(t, x, u(t, x)) \in L_{\mathrm{loc}}^{1}\left(\bar{\Pi}_{T}\right)$.

Если в условии (8) пробные функции из $C_{0}^{\infty}\left(\Pi_{T}\right)$, то немедленно получим

$$
|u-k|_{t}+\operatorname{div}_{x}[\operatorname{sign}(u-k)(\varphi(u)-\varphi(k))] \leqslant \operatorname{sign}(u-k) g \text { в } \mathscr{D}^{\prime}\left(\Pi_{T}\right)
$$

и $u$ удовлетворяет требованию (5). Остается проверить выполнение начального условия. Выберем функцию $\rho(x) \in C_{0}^{\infty}\left(\mathbb{R}^{n}\right), \rho(x) \geqslant 0$, и положим в (8) $f=$ $f_{\nu}(t, x)=\rho(x)\left(1-\theta_{\nu}\left(t-t_{0}\right)\right)$, где $\nu \in \mathbb{N}, t_{0} \in(0, T), 1 / \nu<T-t_{0}$. Получим, что для всех $k \in \mathbb{R}$

$$
\begin{aligned}
\int_{\mathbb{R}^{n}}\left|u_{0}(x)-k\right| \rho(x) d x-\int_{0}^{T} \delta_{\nu}\left(t-t_{0}\right) \int_{\mathbb{R}^{n}}|u(t, x)-k| \rho(x) d x d t \\
\quad+\int_{\Pi_{T}} \operatorname{sign}(u-k)\left[\left(\varphi(u)-\varphi(k), \nabla_{x} \rho\right)+g \rho\right]\left(1-\theta_{\nu}\left(t-t_{0}\right)\right) d t d x \geqslant 0 .
\end{aligned}
$$

Пусть $\mathscr{E}$ - множество общих точек Лебега для функций

$$
t \mapsto \int_{\mathbb{R}^{n}}|u(t, x)-k| \rho(x) d x, \quad k \in \mathbb{R} .
$$

Так же, как и для множества $\mathscr{E} 1$, легко доказать, что $\mathscr{E}$ имеет полную меру Лебега на $(0, T)$, и если $t_{0} \in \mathscr{E}$, то в пределе при $\nu \rightarrow \infty$ из (10) следует соотношение

$$
\begin{aligned}
& \int_{\mathbb{R}^{n}}\left|u_{0}(x)-k\right| \rho(x) d x-\int_{\mathbb{R}^{n}}\left|u\left(t_{0}, x\right)-k\right| \rho(x) d x \\
& \quad+\int_{\Pi_{t_{0}}} \operatorname{sign}(u-k)\left[\left(\varphi(u)-\varphi(k), \nabla_{x} \rho\right)+g \rho\right] d t d x \geqslant 0,
\end{aligned}
$$

которое дает оценку

$$
\int_{\mathbb{R}^{n}}\left|u\left(t_{0}, x\right)-k\right| \rho(x) d x \leqslant \int_{\mathbb{R}^{n}}\left|u_{0}(x)-k\right| \rho(x) d x+C\left(t_{0}\right),
$$


где

$$
C\left(t_{0}\right)=\int_{\Pi_{t_{0}}}\left[|\varphi(u)-\varphi(k)|\left|\nabla_{x} \rho\right|+|g(t, x, u(t, x))| \rho(x)\right] d t d x \underset{t_{0} \in \mathscr{E}, t_{0} \rightarrow 0+}{\longrightarrow} 0 .
$$

Поэтому для любых $k \in \mathbb{R}$ и $\rho(x) \in C_{0}^{\infty}\left(\mathbb{R}^{n}\right), \rho(x) \geqslant 0$, имеем

$$
\limsup _{t_{0} \in \mathscr{E}, t_{0} \rightarrow 0+} \int_{\mathbb{R}^{n}}\left|u\left(t_{0}, x\right)-k\right| \rho(x) d x \leqslant \int_{\mathbb{R}^{n}}\left|u_{0}(x)-k\right| \rho(x) d x .
$$

Пусть $K=\operatorname{supp} \rho(x), k(x) \in C(K)$. Очевидно, для любого положительного $\varepsilon$ можно найти конечный набор функций $h_{i}(x) \in C_{0}^{\infty}\left(\mathbb{R}^{n}\right)$ и последовательность констант $k_{i}, i=1, \ldots, N$, таких, что $h_{i}(x) \geqslant 0, \sum_{i=1}^{N} h_{i}(x)=1$, на компакте $K$ и $\sum_{i=1}^{N}\left|k(x)-k_{i}\right| h_{i}(x) \leqslant \varepsilon \forall x \in K$. Тогда

$$
\begin{aligned}
\int_{\mathbb{R}^{n}}\left|u\left(t_{0}, x\right)-k(x)\right| \rho(x) d x \leqslant & \int_{\mathbb{R}^{n}} \sum_{i=1}^{N}\left|k(x)-k_{i}\right| h_{i}(x) \rho(x) d x \\
& +\int_{\mathbb{R}^{n}} \sum_{i=1}^{N}\left|u\left(t_{0}, x\right)-k_{i}\right| h_{i}(x) \rho(x) d x \\
\leqslant & \varepsilon\|\rho(x)\|_{1}+\int_{\mathbb{R}^{n}} \sum_{i=1}^{N}\left|u\left(t_{0}, x\right)-k_{i}\right| h_{i}(x) \rho(x) d x
\end{aligned}
$$

и ввиду (11)

$$
\begin{aligned}
\limsup _{t_{0} \in \mathscr{E}, t_{0} \rightarrow 0+} \int_{\mathbb{R}^{n}}\left|u\left(t_{0}, x\right)-k(x)\right| \rho(x) d x \\
\leqslant \varepsilon\|\rho(x)\|_{1}+\int_{\mathbb{R}^{n}} \sum_{i=1}^{N}\left|u_{0}(x)-k_{i}\right| h_{i}(x) \rho(x) d x \\
\leqslant 2 \varepsilon\|\rho(x)\|_{1}+\int_{\mathbb{R}^{n}}\left|u_{0}(x)-k(x)\right| \rho(x) d x .
\end{aligned}
$$

Из произвольности $\varepsilon>0$ получаем соотношение

$$
\limsup _{t_{0} \in \mathscr{E}, t_{0} \rightarrow 0+} \int_{\mathbb{R}^{n}}\left|u\left(t_{0}, x\right)-k(x)\right| \rho(x) d x \leqslant \int_{\mathbb{R}^{n}}\left|u_{0}(x)-k(x)\right| \rho(x) d x,
$$

справедливое для любой непрерывной на $K$ функции $k(x)$. Выберем при положительном $\varepsilon$ непрерывную функцию $u_{\varepsilon}$ такую, что $\left\|u_{0}-u_{\varepsilon}\right\|_{1}<\varepsilon$, где $\|\cdot\|_{1}-$ норма в пространстве $L^{1}\left(\mathbb{R}^{n}, \rho(x) d x\right)$. Тогда

$$
\int_{\mathbb{R}^{n}}\left|u\left(t_{0}, x\right)-u_{0}(x)\right| \rho(x) d x \leqslant \varepsilon+\int_{\mathbb{R}^{n}}\left|u\left(t_{0}, x\right)-u_{\varepsilon}(x)\right| \rho(x) d x
$$

и по предельному соотношению (12)

$$
\limsup _{t_{0} \in \mathscr{E}, t_{0} \rightarrow 0+} \int_{\mathbb{R}^{n}}\left|u\left(t_{0}, x\right)-u_{0}(x)\right| \rho(x) d x \leqslant \varepsilon+\int_{\mathbb{R}^{n}}\left|u_{0}(x)-u_{\varepsilon}(x)\right| \rho(x) d x \leqslant 2 \varepsilon
$$

для любого $\varepsilon>0$. Поэтому для любого $\rho(x) \in C_{0}^{\infty}\left(\mathbb{R}^{n}\right), \rho(x) \geqslant 0$,

$$
\limsup _{t_{0} \in \mathscr{E}, t_{0} \rightarrow 0+} \int_{\mathbb{R}^{n}}\left|u\left(t_{0}, x\right)-u_{0}(x)\right| \rho(x) d x=0
$$

и $\underset{t \rightarrow 0+}{\operatorname{ess}} \lim _{t \rightarrow 0} u(t, \cdot)=u_{0}$ в $L_{\text {lос }}^{1}\left(\mathbb{R}^{n}\right)$. Итак, $u$ является о.э.р. задачи $(1),(2)$, что и требовалось доказать. 
СлЕДСТВИЕ 1. Если функиия $u=u(t, x) \in L_{\text {loc }}^{1}\left(\bar{\Pi}_{T}\right)$ является о.ә.р. задачи (1), (2), mо $g=g(t, x, u(t, x)) \in L_{\mathrm{loc}}^{1}\left(\bar{\Pi}_{T}\right)$ и для любой пробной функиии $f=$ $f(t, x) \in C_{0}^{\infty}\left(\bar{\Pi}_{T}\right)$

$$
\int_{\Pi_{T}}\left[u f_{t}+\left(\varphi(u), \nabla_{x} f\right)+g f\right] d t d x+\int_{\mathbb{R}^{n}} u_{0}(x) f(0, x) d x=0 .
$$

ДокАЗАТЕльСтво. То, что $g \in L_{\text {loc }}^{1}\left(\bar{\Pi}_{T}\right)$, было установлено при доказательстве предложения 1. Пусть $f=f(t, x) \in C_{0}^{\infty}\left(\bar{\Pi}_{T}\right), f \geqslant 0, K=\operatorname{supp} f, K_{0}=$ $\left\{x \in \mathbb{R}^{n} \mid(0, x) \in K\right\}=\operatorname{supp} f(0, x)$. Рассмотрим при $m \in \mathbb{N}$ множества $A_{m}=\{(t, x) \in K \mid u(t, x)>-m\}, A_{0, m}=\left\{x \in K_{0} \mid u_{0}(x)>-m\right\}$. Oчeвидно, $A_{m} \subset A_{m+1}, A_{0, m} \subset A_{0, m+1}, \bigcup_{m=1}^{\infty} A_{m}=K \backslash E, \bigcup_{m=1}^{\infty} A_{0, m}=K_{0} \backslash E_{0}$ для любого $m \in \mathbb{N}$, где $E \subset K, E_{0} \subset K_{0}$ - множества нулевой меры Лебега. Полагая в (8) $k=-m$, получим

$$
\begin{aligned}
\int_{A_{m}}\{u & \left.f_{t}+\left(\varphi(u), \nabla_{x} f\right)+g f\right\} d t d x \\
& +\int_{A_{0, m}} u_{0}(x) f(0, x) d x+\int_{A_{m}}\left\{m f_{t}-\left(\varphi(-m), \nabla_{x} f\right)\right\} d t d x \\
& +\int_{A_{0, m}} m f(0, x) d x \\
& -\int_{K \backslash A_{m}}\left\{(u+m) f_{t}+\left(\varphi(u)-\varphi(-m), \nabla_{x} f\right)+g f\right\} d t d x \\
& -\int_{K_{0} \backslash A_{0, m}}\left(u_{0}(x)+m\right) f(0, x) d x \geqslant 0 .
\end{aligned}
$$

Из компактности множеств $K, K_{0}$ следует, что подынтегральные функции в (14) суммируемы. Очевидно, существуют пределы

$$
\begin{aligned}
\lim _{m \rightarrow \infty} \int_{A_{m}}\left\{u f_{t}+\left(\varphi(u), \nabla_{x} f\right)+g f\right\} d t d x+\int_{A_{0, m}} u_{0}(x) f(0, x) d x \\
\quad=\int_{\Pi_{T}}\left\{u f_{t}+\left(\varphi(u), \nabla_{x} f\right)+g f\right\} d t d x+\int_{\mathbb{R}^{n}} u_{0}(x) f(0, x) d x \\
\lim _{m \rightarrow \infty} \int_{K \backslash A_{m}}\left\{u f_{t}+\left(\varphi(u), \nabla_{x} f\right)+g f\right\} d t d x+\int_{K_{0} \backslash A_{0, m}} u_{0}(x) f(0, x) d x=0 .
\end{aligned}
$$

Далее, как следует из формулы интегрирования по частям,

$$
\int_{\Pi_{T}}\left\{m f_{t}-\left(\varphi(-m), \nabla_{x} f\right)\right\} d t d x+\int_{\mathbb{R}^{n}} m f(0, x) d x=0
$$

и, следовательно,

$$
\begin{aligned}
\int_{A_{m}}\left\{m f_{t}-\left(\varphi(-m), \nabla_{x} f\right)\right\} d t d x+\int_{A_{0, m}} m f(0, x) d x \\
\quad=-\int_{K \backslash A_{m}}\left\{m f_{t}-\left(\varphi(-m), \nabla_{x} f\right)\right\} d t d x-\int_{K_{0} \backslash A_{0, m}} m f(0, x) d x .
\end{aligned}
$$


Так как $u(t, x) \in L^{1}(K), u_{0}(x) \in L^{1}\left(K_{0}\right)$, то $\left(\mu_{r}\right.$-мера Лебега на $\left.\mathbb{R}^{r}, r \in \mathbb{N}\right)$

$$
\begin{aligned}
& \mu_{n+1}\left(K \backslash A_{m}\right) \leqslant \frac{1}{m} \int_{K \backslash A_{m}}|u(t, x)| d t d x \leqslant \frac{\alpha_{m}}{m} ; \\
& \mu_{n}\left(K_{0} \backslash A_{0, m}\right) \leqslant \frac{1}{m} \int_{K_{0} \backslash A_{0, m}}\left|u_{0}(x)\right| d x \leqslant \frac{\alpha_{m}}{m},
\end{aligned}
$$

где

$\alpha_{m}=\int_{K} \chi_{|u(t, x)| \geqslant m}|u(t, x)| d t d x+\int_{K_{0}} \chi_{\left|u_{0}(x)\right| \geqslant m}\left|u_{0}(x)\right| d x \rightarrow 0$ при $m \rightarrow \infty$.

Далее, $|\varphi(-m)| \leqslant C_{0}(m+1)$ ввиду (3) и

$$
\begin{aligned}
\left|\int_{K \backslash A_{m}}\left\{m f_{t}-\left(\varphi(-m), \nabla_{x} f\right)\right\} d t d x\right| & \leqslant C(m+|\varphi(-m)|) \mu_{n+1}\left(K \backslash A_{m}\right) \\
& \leqslant C(1+m) \alpha_{m} / m \underset{m \rightarrow \infty}{\longrightarrow} 0, \\
\left|\int_{K_{0} \backslash A_{0, m}} m f(0, x) d x\right| & \leqslant C \alpha_{m} \underset{m \rightarrow \infty}{\longrightarrow} 0
\end{aligned}
$$

где $C$ - постоянная. С учетом соотношений (15)-(19) из (14) в пределе при $m \rightarrow \infty$ вытекает, что

$$
\int_{\Pi_{T}}\left\{u f_{t}+\left(\varphi(u), \nabla_{x} f\right)+g f\right\} d t d x+\int_{\mathbb{R}^{n}} u_{0}(x) f(0, x) d x \geqslant 0 .
$$

Пусть в (8) $k=m \in \mathbb{N}$. Используя соотношения, аналогичные (15)-(19) (с $\left.A_{m}=\{(t, x) \in K \mid u(t, x)<m\}, A_{0, m}=\left\{x \in K_{0} \mid u_{0}(x)<m\right\}\right)$, получим в пределе при $m \rightarrow \infty$, что

$$
-\left\{\int_{\Pi_{T}}\left\{u f_{t}+\left(\varphi(u), \nabla_{x} f\right)+g f\right\} d t d x+\int_{\mathbb{R}^{n}} u_{0}(x) f(0, x) d x\right\} \geqslant 0 .
$$

Итак, для любой функции $f=f(t, x) \in C_{0}^{\infty}\left(\bar{\Pi}_{T}\right), f \geqslant 0$,

$$
\int_{\Pi_{T}}\left\{u f_{t}+\left[\left(\varphi(u), \nabla_{x} f\right)+g f\right]\right\} d t d x+\int_{\mathbb{R}^{n}} u_{0}(x) f(0, x) d x=0 .
$$

Для завершения доказательства остается лишь заметить, что произвольная функция $f(t, x) \in C_{0}^{\infty}\left(\bar{\Pi}_{T}\right)$ равна разности двух неотрицательных функций из $C_{0}^{\infty}\left(\bar{\Pi}_{T}\right)$. Действительно, $f=(f+C) \theta-C \theta$, где константа $C>\|f\|_{\infty}$ и неотрицательная функция $\theta \in C_{0}^{\infty}\left(\bar{\Pi}_{T}\right)$ равна единище на носителе функции $f$.

ЗАмечание 1 . Из тождества (13) при $f=f(t, x) \in C_{0}^{\infty}\left(\Pi_{T}\right)$ вытекает, что

$$
u_{t}+\operatorname{div}_{x} \varphi(u)=g \text { в } \mathscr{D}^{\prime}\left(\Pi_{T}\right)
$$

и $u(t, x)$ удовлетворяет уравнению (1) в смысле распределений (т.е. действительно является обобщенным решением).

Нам понадобятся понятия обобщенного энтропийного суб- и суперрешения (см. [2]-[7], [14]). 
ОПРЕДЕЛЕНИЕ 2 . Функция $u(t, x) \in L_{\text {lос }}^{1}\left(\Pi_{T}\right)$ назьвается обобщенны.м әнтропийным субрешением (коротко, о.э.субр.) задачи Коши (1), (2), если:

1) $\forall k \in \mathbb{R}$ функция $\operatorname{sign}^{+}(u(t, x)-k) g(t, x, u(t, x))$ принадлежит $L_{\text {loc }}^{1}\left(\Pi_{T}\right)$ и

$$
\begin{gathered}
\left((u-k)^{+}\right)_{t}+\operatorname{div}_{x}\left[\operatorname{sign}^{+}(u-k)(\varphi(u)-\varphi(k))\right] \\
\leqslant \operatorname{sign}^{+}(u-k) g(t, x, u) \quad \text { в } \quad \mathscr{D}^{\prime}\left(\Pi_{T}\right)
\end{gathered}
$$

2) ess $\lim _{t \rightarrow 0+}\left(u(t, x)-u_{0}(x)\right)^{+}=0$ в $L_{\text {loc }}^{1}\left(\mathbb{R}^{n}\right)$.

ОПРЕДЕЛЕНИЕ 3. Функция $u(t, x) \in L_{\text {loc }}^{1}\left(\Pi_{T}\right)$ называется обобщенны.м әнтропийным суперрешением (коротко, о.ә.суперр.) задачи Коши (1), (2), если:

1) $\forall k \in \mathbb{R}$ функция $\operatorname{sign}^{-}(u(t, x)-k) g(t, x, u(t, x))$ принадлежит $L_{\text {loc }}^{1}\left(\Pi_{T}\right)$ и

$$
\begin{gathered}
\left((u-k)^{-}\right)_{t}+\operatorname{div}_{x}\left[\operatorname{sign}^{-}(u-k)(\varphi(u)-\varphi(k))\right] \\
\leqslant \operatorname{sign}^{-}(u-k) g(t, x, u) \quad \text { в } \mathscr{D}^{\prime}\left(\Pi_{T}\right)
\end{gathered}
$$

2) ess $\lim _{t \rightarrow 0+}\left(u(t, x)-u_{0}(x)\right)^{-}=0$ в $L_{\text {loc }}^{1}\left(\mathbb{R}^{n}\right)$.

ЗАМЕчАнИЕ 2. а) В общем случае для о.э.субр. (или о.э.суперр.) $u=u(t, x)$ функция $g(t, x, u)$ может не принадлежать $L_{\text {loc }}^{1}\left(\Pi_{T}\right)$. Например, при $\tau \in(0, T)$ функция

$$
u(t, x)= \begin{cases}0, & t \leqslant \tau, \\ -(2(t-\tau))^{-1 / 2}, & t>\tau,\end{cases}
$$

является о.э.субр. задачи Коши $u_{t}=-u^{3}, u_{0}(x)=0$ и, очевидно, $u^{3} \notin L_{\text {loc }}^{1}\left(\Pi_{T}\right)$.

b) Как вытекает из определений, функция $u=u(t, x) \in L^{\infty}\left(\Pi_{T}\right)$ является о.э.суперр. задачи (1), (2) тогда и только тогда, когда функция $-u$ является о.э.субр. задачи Коши для уравнения $u_{t}-\operatorname{div}_{x} \varphi(-u)=-g(t, x,-u)$ с начальными данными $-u_{0}(x)$.

c) Функция $u(t, x) \in L^{\infty}\left(\Pi_{T}\right)$ - о.э.р. задачи $(1),(2)$ тогда и только тогда, когда она является о.э.субр. и о.э.суперр. этой задачи одновременно. Действительно, если $u(t, x)$ - о.э.р. задачи $(1),(2)$, то $g(t, x, u) \in L_{\text {loc }}^{1}\left(\Pi_{T}\right)$ и для любого $k \in \mathbb{R}$

$$
l_{1}=|u-k|_{t}+\operatorname{div}_{x}[\operatorname{sign}(u-k)(\varphi(u)-\varphi(k))]-\operatorname{sign}(u-k) g \leqslant 0 \quad \text { в } \quad \mathscr{D}^{\prime}\left(\Pi_{T}\right) .
$$

Далее, по следствию 1

$$
l_{2}=(u-k)_{t}+\operatorname{div}_{x}(\varphi(u)-\varphi(k))-g=0 \quad \text { в } \mathscr{D}^{\prime}\left(\Pi_{T}\right)
$$

и, значит, распределения $l_{1} \pm l_{2} \leqslant 0$. Поскольку $|r| \pm r=2 r^{ \pm}, \operatorname{sign} r \pm 1=$ $2 \operatorname{sign}^{ \pm} r$, получаем, что вьполнены требования (20) и (21). Начальные условия 2) определений 2,3 также выполнены ввиду оценок $\left(u-u_{0}\right)^{ \pm} \leqslant\left|u-u_{0}\right|$. Итак, $u(t, x)-$ о.э.субр. и о.э.суперр. задачи (1), (2) одновременно.

Обратно, предположим, что $u(t, x)$ - о.э.субр. и о.э.суперр. задачи $(1),(2)$. Из требований $\operatorname{sign}^{ \pm}(u-k) g \in L_{\text {loc }}^{1}\left(\Pi_{T}\right)$ сразу следует, что $g=g(t, x, u) \in L_{\text {loc }}^{1}\left(\Pi_{T}\right)$. Суммируя неравенства (20) и (21), получим соотношение (5). Далее, начальное условие $\mathrm{b}$ ) определения 1 следует из равенства $\left|u-u_{0}\right|=\left(u-u_{0}\right)^{+}+\left(u-u_{0}\right)^{-}$и предельных соотношений в определениях 2 и 3 . Итак, $u(t, x)-$ о.э.р. задачи $(1),(2)$. 
ЗАмЕчАниЕ 3 . Так же, как в предложении 1 и следствии 1 , доказываются следующие утверждения:

1) функция $u=u(t, x) \in L_{\text {loc }}^{1}\left(\bar{\Pi}_{T}\right)$ является о.э.субр. задачи $(1),(2)$ тогда и только тогда, когда для любого $k \in \mathbb{R}$ и любой пробной функции $f=f(t, x) \in$ $C_{0}^{\infty}\left(\bar{\Pi}_{T}\right), f \geqslant 0$,

$$
\begin{gathered}
\int_{\Pi_{T}}\left\{(u-k)^{+} f_{t}+\operatorname{sign}^{+}(u-k)\left[\left(\varphi(u)-\varphi(k), \nabla_{x} f\right)+g f\right]\right\} d t d x \\
+\int_{\mathbb{R}^{n}}\left(u_{0}(x)-k\right)^{+} f(0, x) d x \geqslant 0
\end{gathered}
$$

при этом если $g^{+}(t, x, u(t, x)) \in L_{\text {loc }}^{1}\left(\bar{\Pi}_{T}\right)$, то из $(22)$ в пределе при $k \rightarrow-\infty$ следует, что для любой функции $f \in C_{0}^{\infty}\left(\bar{\Pi}_{T}\right), f \geqslant 0$,

$$
\int_{\Pi_{T}}\left[u f_{t}+\left(\varphi(u), \nabla_{x} f\right)+g f\right] d t d x+\int_{\mathbb{R}^{n}} u_{0}(x) f(0, x) d x \geqslant 0
$$

2) функция $u=u(t, x) \in L_{\text {loc }}^{1}\left(\bar{\Pi}_{T}\right)$ является о.э.суперр. задачи (1), (2) тогда и только тогда, когда для любого $k \in \mathbb{R}$ и любой пробной функции $f=f(t, x) \in$ $C_{0}^{\infty}\left(\bar{\Pi}_{T}\right), f \geqslant 0$,

$$
\begin{gathered}
\int_{\Pi_{T}}\left\{(u-k)^{-} f_{t}+\operatorname{sign}^{-}(u-k)\left[\left(\varphi(u)-\varphi(k), \nabla_{x} f\right)+g f\right]\right\} d t d x \\
\quad+\int_{\mathbb{R}^{n}}\left(u_{0}(x)-k\right)^{-} f(0, x) d x \geqslant 0
\end{gathered}
$$

при этом если $g^{-}(t, x, u(t, x)) \in L_{\text {loc }}^{1}\left(\bar{\Pi}_{T}\right)$, то из (24) в пределе при $k \rightarrow+\infty$ следует, что для любой функции $f \in C_{0}^{\infty}\left(\bar{\Pi}_{T}\right), f \geqslant 0$,

$$
\int_{\Pi_{T}}\left[u f_{t}+\left(\varphi(u), \nabla_{x} f\right)+g f\right] d t d x+\int_{\mathbb{R}^{n}} u_{0}(x) f(0, x) d x \leqslant 0 .
$$

Нам будет полезно также следующее простое

ПРЕДЛОЖЕНИЕ 2. Достаточно, чтобы условия (5), (20) и (21) в определениях 1-3, а также неравенства (8), (22) и (24) выполнялись для всюду плотного множества значений $k \in \mathbb{R}$.

ДОКАЗАТЕЛЬСТво. Пусть $D \subset \mathbb{R}$-всюду плотное подмножество и условие (20) выполняется для всех $k \in D$. Выберем произвольное $k \in \mathbb{R}$, и пусть $\beta \in D, \beta>k$. По условию при $k=\beta$ вьполнено (20), т.е. для любой функции $f=f(t, x) \in$ $C_{0}^{\infty}\left(\Pi_{T}\right), f \geqslant 0$,

$$
\int_{\Pi_{T}}\left\{(u-\beta)^{+} f_{t}+\operatorname{sign}^{+}(u-\beta)\left[\left(\varphi(u)-\varphi(\beta), \nabla_{x} f\right)+g f\right]\right\} d t d x \geqslant 0 .
$$


Переходя в этом неравенстве к пределу при $\beta \rightarrow k$ и учитывая, что $\operatorname{sign}^{+}(u-$ $\beta) \rightarrow \operatorname{sign}^{+}(u-k)$ для всех $u \in \mathbb{R}$, получаем, что для любой функции $f=f(t, x) \in$ $C_{0}^{\infty}\left(\Pi_{T}\right), f \geqslant 0$,

$$
\int_{\Pi_{T}}\left\{(u-k)^{+} f_{t}+\operatorname{sign}^{+}(u-k)\left[\left(\varphi(u)-\varphi(k), \nabla_{x} f\right)+g f\right]\right\} d t d x \geqslant 0,
$$

т.е. (20) выполнено для всех $k \in \mathbb{R}$.

Условие (21) разбирается аналогично. Нужно лишь выбрать значения $\beta \in D$, $\beta<k$, рассмотреть интегральное неравенство, соответствующее (21) при $k=\beta$, и перейти к пределу при $\beta \rightarrow k$. Наконец, если для всех $k \in D$ выполнено условие (5), то рассмотрим сумму соотношений (5) при $k=\alpha$ и $k=\beta$, где $\alpha, \beta \in D, \alpha<k<\beta$. Учитывая, что $\forall u \in \mathbb{R}$ при $\alpha, \beta \rightarrow k$

$$
\begin{gathered}
|u-\alpha|+|u-\beta| \rightarrow 2|u-k|, \\
\operatorname{sign}(u-\alpha)(\varphi(u)-\varphi(\alpha))+\operatorname{sign}(u-\beta)(\varphi(u)-\varphi(\beta)) \rightarrow \\
\rightarrow 2 \operatorname{sign}(u-k)(\varphi(u)-\varphi(k)), \\
\operatorname{sign}(u-\alpha)+\operatorname{sign}(u-\beta) \rightarrow 2 \operatorname{sign}(u-k),
\end{gathered}
$$

получаем, что (5) справедливо для всех вещественных $k$. Утверждение, касающееся неравенств (8), (22) и (24), доказывается на базе тех же предельных процедур. Предложение доказано.

\section{§ 2. Существование о.э.p.}

Для доказательства существования о.э.р. нам понадобится следующая априорная оценка для о.э.субр. и о.э.суперр. задачи (1), (2). Пусть $p(s) \in C^{\infty}(\mathbb{R})-$ невозрастаюшая функция такая, что $p(s)=1$ при $s \leqslant 0$ и $p(s)=0$ при $s \geqslant 1$. Справедливо

ПРЕДЛОЖЕНИЕ 3. Пусть $u=u(t, x)$ - о.ә.субр. задачи (1), (2), $R>0$. Тогда для п.в. $\tau \in(0, T)$

$$
\begin{aligned}
\int_{\mathbb{R}^{n}} u^{+}(\tau, x) p(|x|-R) d x \leqslant e^{L \tau} & \left(\int_{\mathbb{R}^{n}}\left(u_{0}^{+}(x)+2\right) p\left(|x|-C_{0} \tau-R\right) d x\right. \\
& \left.+\int_{\Pi_{\tau}} g^{+}(t, x, 0) p\left(|x|-C_{0}(\tau-t)-R\right) d t d x\right)
\end{aligned}
$$

Если же $u=u(t, x)$ - о.э.суперр. задачи (1), (2), то для п.в. $\tau \in(0, T)$

$$
\begin{aligned}
\int_{\mathbb{R}^{n}} u^{-}(\tau, x) p(|x|-R) d x \leqslant e^{L \tau} & \left(\int_{\mathbb{R}^{n}}\left(u_{0}^{-}(x)+2\right) p\left(|x|-C_{0} \tau-R\right) d x\right. \\
& \left.+\int_{\Pi_{\tau}} g^{-}(t, x, 0) p\left(|x|-C_{0}(\tau-t)-R\right) d t d x\right)_{(2)}
\end{aligned}
$$


ДокАЗАТЕЛЬСТво. Пусть $f(t, x)=p\left(|x|-R-C_{0}\left(t_{0}-t\right)\right) \theta_{\nu}(\tau-t)$, где $0<$ $\tau<t_{0}<T$, функция $\theta_{\nu}$, аппроксимирующая функцию Хевисайда, определена при доказательстве предложения 1 . Тогда $f=f(t, x) \in C_{0}^{\infty}\left(\bar{\Pi}_{T}\right), f \geqslant 0$, и по замечанию 3,1$)$ при $k=0, g=g(t, x, u(t, x))$

$\int_{\Pi_{T}}\left\{u^{+} f_{t}+\operatorname{sign}^{+}(u)\left[\left(\varphi(u)-\varphi(0), \nabla_{x} f\right)+g f\right]\right\} d t d x+\int_{\mathbb{R}^{n}}\left(u_{0}(x)\right)^{+} f(0, x) d x \geqslant 0$.

Добавляя удвоенное равенство $\int_{\Pi_{T}} f_{t} d t d x+\int_{\mathbb{R}^{n}} f(0, x) d x=0$, получим

$$
\begin{gathered}
\int_{\Pi_{T}}\left\{\left(u^{+}+2\right) f_{t}+\operatorname{sign}^{+}(u)\left[\left(\varphi(u)-\varphi(0), \nabla_{x} f\right)+g f\right]\right\} d t d x \\
+\int_{\mathbb{R}^{n}}\left(\left(u_{0}(x)\right)^{+}+2\right) f(0, x) d x \geqslant 0,
\end{gathered}
$$

т.е. при $\rho(t, x)=p\left(|x|-R-C_{0}\left(t_{0}-t\right)\right), 1 / \nu<\tau$

$$
\begin{aligned}
& -\int_{\Pi_{T}}\left(u^{+}+2\right) \rho(t, x) \delta_{\nu}(\tau-t) d t d x \\
& \quad+\int_{\Pi_{T}}\left\{\left(u^{+}+2\right) \rho_{t}+\operatorname{sign}^{+}(u)\left[\left(\varphi(u)-\varphi(0), \nabla_{x} \rho\right)+g \rho\right] \theta_{\nu}(\tau-t)\right\} d t d x \\
& \quad+\int_{\mathbb{R}^{n}}\left(\left(u_{0}(x)\right)^{+}+2\right) \rho(0, x) d x \geqslant 0
\end{aligned}
$$

Из условия (3) следует, что $\operatorname{sign}^{+}(u)|\varphi(u)-\varphi(0)| \leqslant C_{0}\left(u^{+}+2\right)$, и с учетом равенства $\rho_{t}=-C_{0}\left|\nabla_{x} \rho\right|$

$$
\begin{aligned}
& \left(u^{+}+2\right) \rho_{t}+\operatorname{sign}^{+}(u)\left(\varphi(u)-\varphi(0), \nabla_{x} \rho\right) \\
& \quad \leqslant\left(u^{+}+2\right) \rho_{t}+\operatorname{sign}^{+}(u)|\varphi(u)-\varphi(0)| \cdot\left|\nabla_{x} \rho\right| \\
& \quad \leqslant\left(u^{+}+2\right)\left(\rho_{t}+C_{0}\left|\nabla_{x} \rho\right|\right)=0 .
\end{aligned}
$$

Поэтому из (28) следует оценка

$$
\begin{array}{r}
\int_{\Pi_{T}}\left(u^{+}+2\right) \rho(t, x) \delta_{\nu}(\tau-t) d t d x \leqslant \int_{\mathbb{R}^{n}}\left(\left(u_{0}(x)\right)^{+}+2\right) \rho(0, x) d x \\
\quad+\int_{\Pi_{T}}\left(g^{+}(t, x, 0)+L\left(u^{+}(t, x)+2\right)\right) \rho(t, x) \theta_{\nu}(\tau-t) d t d x
\end{array}
$$

Мы использовали также неравенство

$$
\begin{aligned}
\operatorname{sign}^{+}(u) g(t, x, u) & \leqslant g^{+}(t, x, 0)+\operatorname{sign}^{+}(u)(g(t, x, u)-g(t, x, 0)) \\
& \leqslant g^{+}(t, x, 0)+L u^{+} \leqslant g^{+}(t, x, 0)+L\left(u^{+}+2\right),
\end{aligned}
$$

вытекающее из (4). Пусть $\mathscr{E}$ - множество полной меры общих точек Лебега счетного семейства функций $t \mapsto \int_{\mathbb{R}^{n}}\left(u^{+}(t, x)+2\right) \rho(t, x) d x$, где $R, t_{0} \in \mathbb{Q}$. Если $\tau \in \mathscr{E}$, то из (29) в пределе при $\nu \rightarrow \infty$ следует неравенство

$$
\begin{aligned}
\int_{\mathbb{R}^{n}}\left(u^{+}(\tau, x)+2\right) \rho(\tau, x) d x \leqslant \int_{\mathbb{R}^{n}}\left(\left(u_{0}(x)\right)^{+}+2\right) \rho(0, x) d x \\
\quad+\int_{\Pi_{t_{0}}} g^{+}(t, x, 0) \rho(t, x) d t d x+L \int_{0}^{\tau} \int_{\mathbb{R}^{n}}\left(u^{+}(t, x)+2\right) \rho(t, x) d t d x
\end{aligned}
$$


По лемме Гронуолла тогда для п.в. $\tau \in\left(0, t_{0}\right)$

$$
\begin{array}{r}
\int_{\mathbb{R}^{n}}\left(u^{+}(\tau, x)+2\right) \rho(\tau, x) d x \leqslant e^{L \tau}\left(\int_{\mathbb{R}^{n}}\left(\left(u_{0}(x)\right)^{+}+2\right) \rho(0, x) d x\right. \\
\left.+\int_{\Pi_{t_{0}}} g^{+}(t, x, 0) \rho(t, x) d t d x\right)
\end{array}
$$

для всех рациональных $R>0, t_{0} \in(\tau, T)$. Заметим, что интегралы в последнем неравенстве непрерывно зависят от параметров $R, t_{0}$, и поэтому это неравенство вьполнено для всех $R>0, t_{0} \in(\tau, T)$. Тогда оценка (26) получается из (30) в пределе при $t_{0} \rightarrow \tau+$.

Доказательство оценки (27) аналогично. Нужно лишь воспользоваться замечанием 3,2$)$ с той же пробной функцией $f(t, x)$, что и ранее. Предложение доказано.

Если $u(t, x)$ - о.э.р. задачи $(1),(2)$, то по замечанию 2, с) оно является о.э.суби суперрешением этой задачи. Суммируя соотношения (26) и (27), приходим к следующей оценке.

СЛЕДСТВИЕ 2. Если $u=u(t, x)-$ o.ə.p. задачи (1), (2), $R>0$, mo для n. $\boldsymbol{\theta}$. $\tau \in(0, T)$

$$
\begin{aligned}
\int_{\mathbb{R}^{n}}|u(\tau, x)| p(|x|-R) d x \leqslant e^{L \tau} & \left(\int_{\mathbb{R}^{n}}\left(\left|u_{0}(x)\right|+4\right) p\left(|x|-C_{0} \tau-R\right) d x\right. \\
& \left.+\int_{\Pi_{\tau}}|g(t, x, 0)| p\left(|x|-C_{0}(\tau-t)-R\right) d t d x\right)
\end{aligned}
$$

Нам понадобится следующее простое обобщение известной теоремы Лебега о предельном переходе под знаком интеграла.

Лемма 1. Пусть $\Omega \subset \mathbb{R}^{N}$ - измеримая область; $u_{k}, v_{k} \in L^{1}(\Omega), \quad k \in \mathbb{N},-$ последовательности такие, что $0 \leqslant u_{k}(x) \leqslant v_{k}(x)$ для п.в. $x \in \Omega$, и при $k \rightarrow \infty \quad u_{k} \rightarrow$ и.в. на $\Omega, v_{k} \rightarrow v$ в $L^{1}(\Omega)$. Тогда $u_{k} \rightarrow u$ в $L^{1}(\Omega)$.

ДоКАЗАТЕЛЬСТВО. Заметим, что после выделения подпоследовательности $v_{k} \rightarrow v$ п.в. на $\Omega$ и из неравенств $u_{k}(x) \leqslant v_{k}(x)$ вытекает в пределе при $k \rightarrow \infty$, что $u \leqslant v$ п.в. на $\Omega$.

Положим $w_{k}(x)=\left(\left|u_{k}(x)-u\right|-\left|v_{k}(x)-v(x)\right|\right)^{+}$. Тогда ввиду неравенств

$$
\left|u_{k}-u\right| \leqslant u_{k}+u \leqslant v_{k}+u \leqslant\left|v_{k}-v\right|+v+u \leqslant\left|v_{k}-v\right|+2 v
$$

справедлива оценка $0 \leqslant w_{k}(x) \leqslant 2 v(x) \in L^{1}(\Omega)$ для п.в. $x \in \Omega$. Очевидно, $w_{k}(x) \rightarrow 0$ п.в. на $\Omega$ при $k \rightarrow \infty$, и по теореме Лебега о предельном переходе под знаком интеграла $w_{k}(x) \rightarrow 0$ в $L^{1}(\Omega)$. Ввиду оценки $\left|w_{k}-\right| u_{k}(x)-u|| \leqslant\left|v_{k}-v\right|$, также $w_{k}-\left|u_{k}-u\right| \rightarrow 0$ в $L^{1}(\Omega)$. Поэтому $u_{k}-u \rightarrow 0$ в $L^{1}(\Omega)$, что и требовалось доказать.

Верна следуюшая теорема. 
TЕОРема 1. Пусть $u_{r}=u_{r}(t, x), \quad r \in \mathbb{N},-$ последовательность о.ә.р. (о.э.субр., о.ә.суперр.) задачи (1), (2) с входныцми даннымми $u_{0, r}(x), g_{r}(t, x, u)$, причем при $r \rightarrow \infty \quad u_{0, r} \rightarrow u_{0}=u_{0}(x)$ в $L_{\mathrm{loc}}^{1}\left(\mathbb{R}^{n}\right), \quad u_{r} \rightarrow u=u(t, x)$, $g_{r}(t, x, u) \rightarrow g(t, x, u)$ в $L_{\mathrm{loc}}^{1}\left(\bar{\Pi}_{T}\right)$ для всех $u \in \mathbb{R}$. Предположим также, что $g_{r}(t, x, u)$ удовлетворяют условию (4) с константой $L$, не зависящей от $r$. Тогда и $(t, x)$ - о.ә.р. (соответственно, о.ә.субр., о.ә.суперр.) задачи (1), (2) с входными данными $\left(u_{0}, g\right)$.

ДокАЗАТЕЛЬСТво проведем для случая последовательности о.э.р. Для о.э.субр. и о.э.суперр. доказательство аналогично. Выделяя при необходимости подпоследовательность, будем считать, что при $r \rightarrow \infty u_{r}(t, x) \rightarrow u(t, x)$, $g_{r}(t, x, u) \rightarrow g(t, x, u)$ для любого $u \in \mathbb{Q}$ п.в. на $\Pi_{T}$. По предложению 1 для любого $k \in \mathbb{R}$ и любой пробной функции $f=f(t, x) \in C_{0}^{\infty}\left(\bar{\Pi}_{T}\right), f \geqslant 0$,

$$
\begin{gathered}
\int_{\Pi_{T}}\left\{\left|u_{r}-k\right| f_{t}+\operatorname{sign}\left(u_{r}-k\right)\left[\left(\varphi\left(u_{r}\right)-\varphi(k), \nabla_{x} f\right)+g_{r} f\right]\right\} d t d x \\
\quad+\int_{\mathbb{R}^{n}}\left|u_{0, r}(x)-k\right| f(0, x) d x \geqslant 0,
\end{gathered}
$$

что можно переписать в виде

$$
\begin{gathered}
\int_{\Pi_{T}}\left\{\left|u_{r}-k\right| f_{t}+\operatorname{sign}\left(u_{r}-k\right)\left(\varphi\left(u_{r}\right)-\varphi(k), \nabla_{x} f\right)+\left(\operatorname{sign}\left(u_{r}-k\right) g_{r}\right)^{+} f\right\} d t d x \\
+\int_{\mathbb{R}^{n}}\left|u_{0, r}(x)-k\right| f(0, x) d x \geqslant \int_{\Pi_{T}}\left(\operatorname{sign}\left(u_{r}-k\right) g_{r}\right)^{-} f d t d x
\end{gathered}
$$

Заметим далее, что для п.в. $k \in \mathbb{R}$ множество уровня $\left\{(t, x) \in \Pi_{T} \mid u(t, x)=k\right\}$ имеет нулевую меру Лебега, и поэтому

$$
\operatorname{sign}\left(u_{r}(t, x)-k\right) \underset{r \rightarrow \infty}{\longrightarrow} \operatorname{sign}(u(t, x)-k) \text { п.в. на } \Pi_{T} \text {. }
$$

Покажем, что $g_{r}\left(t, x, u_{r}(t, x)\right) \rightarrow g(t, x, u(t, x))$ при $r \rightarrow \infty$ п.в. на $\Pi_{T}$. Пусть $\mathscr{E}-$ множество полной меры значений $(t, x) \in \Pi_{T}$, для которых при $r \rightarrow \infty u_{r}(t, x) \rightarrow$ $u(t, x), g_{r}(t, x, u) \rightarrow g(t, x, u)$ для любого $u \in \mathbb{Q}$.

Заметим, что ввиду (4) функции $h_{r}(t, x, u)=g_{r}(t, x, u)-L u$ не возрастают и непрерывны по переменной $u \in \mathbb{R}$, и если $(t, x) \in \mathscr{E}$, то $h_{r}(t, x, u) \rightarrow h(t, x, u)=$ $g(t, x, u)-L u$ при $r \rightarrow \infty$ для любого $u \in \mathbb{Q}$. Тогда, учитьвая монотонность функций $h_{r}(t, x, u)$ по последней переменной и непрерывность функции $u \mapsto g(t, x, u)$, выводим, что $h_{r}(t, x, u) \rightarrow h(t, x, u)$ при $r \rightarrow \infty$ для всех $u \in \mathbb{R}$, причем равномерно на компактных подмножествах $\mathbb{R}$, по теореме о равномерной сходимости последовательности монотонных функций. Поскольку также $u_{r}(t, x) \rightarrow u(t, x)$ при $r \rightarrow \infty$, то

$$
h_{r}\left(t, x, u_{r}(t, x)\right) \underset{r \rightarrow \infty}{\longrightarrow} h(t, x, u(t, x))
$$

и

$$
\begin{array}{r}
g_{r}\left(t, x, u_{r}(t, x)\right)=h_{r}\left(t, x, u_{r}(t, x)\right)+L u_{r}(t, x) \underset{r \rightarrow \infty}{\longrightarrow} \\
\underset{r \rightarrow \infty}{\longrightarrow} g(t, x, u(t, x))=h(t, x, u(t, x))+L u(t, x)
\end{array}
$$


для всех $(t, x) \in \mathscr{E}$. Учитывая также (33), получаем, что для п.в. $k \in \mathbb{R}$

$$
\operatorname{sign}\left(u_{r}-k\right) g_{r}\left(t, x, u_{r}(t, x)\right) \rightarrow \operatorname{sign}(u-k) g(t, x, u(t, x)) \text { п.в. на } \Pi_{T} .
$$

Далее, как следует из $(4), \operatorname{sign}\left(u_{r}-k\right)\left(g_{r}\left(t, x, u_{r}\right)-g(t, x, k)\right) \leqslant L\left|u_{r}-k\right|$, откуда вытекает оценка

$$
\left(\operatorname{sign}\left(u_{r}-k\right) g_{r}\left(t, x, u_{r}\right)\right)^{+} f \leqslant\left(\left|g_{r}(t, x, k)\right|+L\left|u_{r}-k\right|\right) f
$$

и, очевидно, $\left(\left|g_{r}(t, x, k)\right|+L\left|u_{r}-k\right|\right) f \rightarrow(|g(t, x, k)|+L|u-k|) f$ в $L^{1}\left(\Pi_{T}\right)$. Тогда по лемме 1 при $r \rightarrow \infty$

$$
\left(\operatorname{sign}\left(u_{r}-k\right) g_{r}\left(t, x, u_{r}\right)\right)^{+} f \rightarrow(\operatorname{sign}(u-k) g(t, x, u))^{+} f \quad \text { в } \quad L^{1}\left(\Pi_{T}\right) ;
$$

здесь $u_{r}=u_{r}(t, x), u=u(t, x)$. Ввиду оценки $\left|\varphi\left(u_{r}\right)-\varphi(k)\right| \leqslant C_{0}\left(\left|u_{r}\right|+|k|+2\right)$, можно снова применить лемму 1 и получить соотношение

$$
\begin{aligned}
& \operatorname{sign}\left(u_{r}-k\right)\left(\varphi\left(u_{r}\right)-\varphi(k), \nabla_{x} f\right) \rightarrow \\
& \quad \rightarrow \operatorname{sign}(u-k)\left(\varphi(u)-\varphi(k), \nabla_{x} f\right) \quad \text { в } \quad L^{1}\left(\Pi_{T}\right) .
\end{aligned}
$$

Учитывая, что $\left|u_{r}-k\right| \rightarrow|u-k|$ в $L_{\text {loc }}^{1}\left(\bar{\Pi}_{T}\right)$ и $\left|u_{0, r}-k\right| \rightarrow\left|u_{0}-k\right|$ в $L_{\text {loc }}^{1}\left(\mathbb{R}^{n}\right)$ при $r \rightarrow \infty$, а также предельные соотношения (34), (35), получим, что для п.в. $k \in \mathbb{R}$ при $r \rightarrow \infty$

$$
\begin{aligned}
\int_{\Pi_{T}}\left\{\left|u_{r}-k\right| f_{t}+\operatorname{sign}\left(u_{r}-k\right)\left(\varphi\left(u_{r}\right)-\varphi(k), \nabla_{x} f\right)+\left(\operatorname{sign}\left(u_{r}-k\right) g_{r}\right)^{+} f\right\} d t d x \\
\quad+\int_{\mathbb{R}^{n}}\left|u_{0, r}(x)-k\right| f(0, x) d x \rightarrow \\
\rightarrow \int_{\Pi_{T}}\left\{|u-k| f_{t}+\operatorname{sign}(u-k)\left(\varphi(u)-\varphi(k), \nabla_{x} f\right)+(\operatorname{sign}(u-k) g)^{+} f\right\} d t d x \\
\quad+\int_{\mathbb{R}^{n}}\left|u_{0}(x)-k\right| f(0, x) d x .
\end{aligned}
$$

Далее, $\left(\operatorname{sign}\left(u_{r}-k\right) g_{r}\right)^{-} \rightarrow(\operatorname{sign}(u-k) g)^{-}$при $r \rightarrow \infty$ п.в. на $\Pi_{T}$. Применяя лемму Фату к интегралу в правой части (32) и используя полученные выше предельные соотношения, получим, что для п.в. $k \in \mathbb{R}$

$$
\begin{aligned}
& \int_{\Pi_{T}}(\operatorname{sign}(u-k) g)^{-} f d t d x \\
& \leqslant \int_{\Pi_{T}}\left\{|u-k| f_{t}+\operatorname{sign}(u-k)\left(\varphi(u)-\varphi(k), \nabla_{x} f\right)\right. \\
& \left.\quad+(\operatorname{sign}(u-k) g)^{+} f\right\} d t d x+\int_{\mathbb{R}^{n}}\left|u_{0}(x)-k\right| f(0, x) d x
\end{aligned}
$$

т.е. для п.в. $k \in \mathbb{R}$ и любой пробной функции $f=f(t, x) \in C_{0}^{\infty}\left(\bar{\Pi}_{T}\right), f \geqslant 0$,

$$
\begin{aligned}
\int_{\Pi_{T}}\left\{|u-k| f_{t}+\operatorname{sign}(u-k)\left[\left(\varphi(u)-\varphi(k), \nabla_{x} f\right)+g f\right]\right\} d t d x \\
+\int_{\mathbb{R}^{n}}\left|u_{0}(x)-k\right| f(0, x) d x \geqslant 0 .
\end{aligned}
$$


По предложениям $1,2 u(t, x)$ - о.э.р. задачи $(1),(2)$ с входными данными $\left(u_{0}, g\right)$. Теорема доказана.

Доказательство существования о.э.р. проведем сначала в случае $g(t, x, u)=$ $g(t, x) \in L_{\mathrm{loc}}^{1}\left(\bar{\Pi}_{T}\right)$. Отметим, что при $g \equiv 0$ приводимая ниже теорема доказана в $[16]$.

TEOPEмA 2. Существует отображение $\mathscr{F}: L_{\mathrm{loc}}^{1}\left(\mathbb{R}^{n}\right) \times L_{\mathrm{loc}}^{1}\left(\bar{\Pi}_{T}\right) \mapsto L_{\mathrm{loc}}^{1}\left(\bar{\Pi}_{T}\right)$, сопоставляющее входным данным $\left(u_{0}, g\right)$ задачи (1), (2) о.ә.р. $u=\mathscr{F}\left(u_{0}, g\right)$ так, что выполнено свойство: если $u=\mathscr{F}\left(u_{0}, g\right), \quad v=\mathscr{F}\left(v_{0}, h\right) u\left(u_{0}-\right.$ $\left.v_{0}\right)^{+} \in L^{1}\left(\mathbb{R}^{n}\right), \quad(g-h)^{+} \in L^{1}\left(\Pi_{T}\right)$, то для п.в. $\tau \in(0, T)$

$$
\begin{aligned}
\int_{\mathbb{R}^{n}}(u(\tau, x)-v(\tau, x))^{+} d x \leqslant & \int_{\mathbb{R}^{n}}\left(u_{0}(x)-v_{0}(x)\right)^{+} d x \\
& +\int_{\Pi_{\tau}} \chi_{u} \geqslant v(g(t, x)-h(t, x))^{+} d t d x .
\end{aligned}
$$

ДокАЗАТЕЛЬСТво. Рассмотрим сначала случай, когда $u_{0} \in L^{\infty}\left(\mathbb{R}^{n}\right) \cap L^{1}\left(\mathbb{R}^{n}\right)$, $g \in L^{\infty}\left(\Pi_{T}\right) \cap L^{1}\left(\Pi_{T}\right)$. Как показано в работе [1], сушествует наибольшее (а также и наименьшее) о.э.р. задачи (1), (2), причем это решение $u \in C\left([0, T], L^{1}\left(\mathbb{R}^{n}\right)\right)$ является мягким решением (mild solution) задачи Коши для соответствуюшего эволюционного уравнения $\dot{u}+A u=g, u(0)=u_{0}$, где $A: D(A) \mapsto L^{1}\left(\mathbb{R}^{n}\right)$, $D(A) \subset L^{1}\left(\mathbb{R}^{n}\right)$ - некоторое $m$-аккретивное и $T$-аккретивное в $L^{1}\left(\mathbb{R}^{n}\right)$ продолжение оператора $u \mapsto \operatorname{div} \varphi(u)$. Положим $u=\mathscr{F}\left(u_{0}, g\right)$. В соответствии с результатами нелинейной теории полугрупп (см. [4], [7]) для двух решений $u=\mathscr{F}\left(u_{0}, g\right)$, $v=\mathscr{F}\left(v_{0}, h\right)$ выполнено неравенство

$$
\left\|(u(\tau, \cdot)-v(\tau, \cdot))^{+}\right\|_{1} \leqslant\left\|\left(u_{0}-v_{0}\right)^{+}\right\|_{1}+\int_{\Pi_{\tau}}[u-v, g-h] d t d x,
$$

где обозначено

$$
[p, q]=\left[\chi_{p>0} q+\chi_{p=0} q^{+}\right]=\lim _{\beta \rightarrow 0+}\left[(p+\beta q)^{+}-p^{+}\right] / \beta .
$$

Заметим, что ввиду выпуклости функции $u \mapsto u^{+}$для всех $\beta>0$

$$
[p, q] \leqslant[p, q]_{\beta}, \quad \text { где }[p, q]_{\beta}=\left[(p+\beta q)^{+}-p^{+}\right] / \beta .
$$

Таким образом,

$$
\begin{aligned}
\int_{\mathbb{R}^{n}}(u(\tau, x)-v(\tau, x))^{+} d x \leqslant \int_{\mathbb{R}^{n}}\left(u_{0}(x)-v_{0}(x)\right)^{+} d x \\
\quad+\int_{\Pi_{\tau}}\left[\chi_{u>v}(g(t, x)-h(t, x))+\chi_{u=v}(g(t, x)-h(t, x))^{+}\right] d t d x \\
\leqslant \int_{\Pi_{\tau}} \chi_{u>v}(g(t, x)-h(t, x))^{+} d t d x
\end{aligned}
$$


т.е. справедливо неравенство (36). Меняя местами функции $u$ и $v$ в (36) и суммируя полученные неравенства, выводим, что для любого $\tau \in(0, T)$

$$
\int_{\mathbb{R}^{n}}|u(\tau, x)-v(\tau, x)| d x \leqslant \int_{\mathbb{R}^{n}}\left|u_{0}(x)-v_{0}(x)\right| d x+\int_{\Pi_{\tau}}|g(t, x)-h(t, x)| d t d x,
$$

откуда следует, что $\left(u_{0}, g\right) \mapsto \mathscr{F}\left(u_{0}, g\right)$ - равномерно непрерьвное отображение из $\left(L^{\infty}\left(\mathbb{R}^{n}\right) \cap L^{1}\left(\mathbb{R}^{n}\right)\right) \times\left(L^{\infty}\left(\Pi_{T}\right) \cap L^{1}\left(\Pi_{T}\right)\right) \subset L^{1}\left(\mathbb{R}^{n}\right) \times L^{1}\left(\Pi_{T}\right)$ в $C\left([0, T], L^{1}\left(\mathbb{R}^{n}\right)\right) \subset$ $L^{1}\left(\Pi_{T}\right)$, однозначно продолжающееся до непрерывного отображения $\mathscr{F}: L^{1}\left(\mathbb{R}^{n}\right) \times$ $L^{1}\left(\Pi_{T}\right) \mapsto L^{1}\left(\Pi_{T}\right)$. Если $u_{0} \in L^{1}\left(\mathbb{R}^{n}\right), g \in L^{1}\left(\Pi_{T}\right)$ и $u=\mathscr{F}\left(u_{0}, g\right)$, то можно выбрать последовательности $u_{k}^{0}(x) \in L^{\infty}\left(\mathbb{R}^{n}\right) \cap L^{1}\left(\mathbb{R}^{n}\right), g_{k} \in L^{\infty}\left(\Pi_{T}\right) \cap L^{1}\left(\Pi_{T}\right)$, $k \in \mathbb{N}$, такие, что $u_{k}^{0} \rightarrow u_{0}$ в $L^{1}\left(\mathbb{R}^{n}\right), g_{k} \rightarrow g$ в $L^{1}\left(\Pi_{T}\right)$ при $k \rightarrow \infty$. Положим $u_{k}=\mathscr{F}\left(u_{k}^{0}, g_{k}\right)$. Тогда $u_{k} \rightarrow u$ в $L^{1}\left(\Pi_{T}\right)$ и по теореме $1 u$ - о.э.р. задачи (1), (2). Как следует из (37), в случае, когда $u_{0}, v_{0} \in L^{\infty}\left(\mathbb{R}^{n}\right) \cap L^{1}\left(\mathbb{R}^{n}\right)$, $g, h \in L^{\infty}\left(\Pi_{T}\right) \cap L^{1}\left(\Pi_{T}\right)$, для о.э.p. $u=\mathscr{F}\left(u_{0}, g\right), u=\mathscr{F}\left(v_{0}, h\right)$ при всех $\beta>0$ справедливо неравенство

$$
\begin{aligned}
\int_{\mathbb{R}^{n}}(u(\tau, x)-v(\tau, x))^{+} d x \leqslant & \int_{\mathbb{R}^{n}}\left(u_{0}(x)-v_{0}(x)\right)^{+} d x \\
& +\int_{\Pi_{\tau}}\left[((u-v)+\beta(g-h))^{+}-(u-v)^{+}\right] / \beta .
\end{aligned}
$$

По непрерьвности это неравенство верно и в общем случае $u_{0}, v_{0} \in L^{1}\left(\mathbb{R}^{n}\right), g, h \in$ $L^{1}\left(\Pi_{T}\right)$. Переходя в $(38)$ к пределу при $\beta \rightarrow 0$, получим $(37)$, что завершает доказательство теоремы в случае суммируемых входных данных.

В общем случае $u_{0}(x) \in L_{\text {loc }}^{1}\left(\mathbb{R}^{n}\right), g(t, x) \in L_{\text {loc }}^{1}\left(\bar{\Pi}_{T}\right)$ положим при $k, r \in \mathbb{N}$

$$
\begin{aligned}
u_{k, r}^{0}(x) & =\left(u_{0}\right)^{+} \chi_{|x|<k}-\left(u_{0}\right)^{-} \chi_{|x|<r} \in L^{1}\left(\mathbb{R}^{n}\right), \\
g_{k, r}(t, x) & =g^{+} \chi_{|x|<k}-g^{-} \chi_{|x|<r} \in L^{1}\left(\Pi_{T}\right), \\
u_{k, r} & =\mathscr{F}_{k, r}\left(u_{0}, g\right)=\mathscr{F}\left(u_{k, r}^{0}, g_{k, r}\right) .
\end{aligned}
$$

Поскольку $u_{k, r+1}^{0} \leqslant u_{k, r}^{0} \leqslant u_{k+1, r}^{0}, g_{k, r+1} \leqslant g_{k, r} \leqslant g_{k+1, r}$ для всех $k, r \in \mathbb{N}$, то, как непосредственно следует из свойства (36),

$$
u_{k, r+1} \leqslant u_{k, r} \leqslant u_{k+1, r} \text { п.в. на } \Pi_{T} .
$$

Поэтому п.в. на П $T$ существуют пределы $u_{r}=u_{r}(t, x)=\lim _{k \rightarrow \infty} u_{k, r}(t, x) \leqslant+\infty$. При этом, ввиду (39),

$$
\forall r \in \mathbb{N} \quad u_{r+1} \leqslant u_{r} \text { п.в. на } \Pi_{T} .
$$

Далее, так как $u_{k, r}(t, x)$ - о.э.р. задачи $(1),(2)$ с соответствуюшими входными данными, то по следствию 2 (при $L=0$ ) для любого $R>0$ и для п.в. $\tau \in(0, T)$

$$
\begin{aligned}
\int_{\mathbb{R}^{n}}\left|u_{k, r}(\tau, x)\right| p(|x|-R) d x \leqslant & \int_{\mathbb{R}^{n}}\left(\left|u_{k, r}^{0}(x)\right|+4\right) p\left(|x|-C_{0} \tau-R\right) d x \\
& +\int_{\Pi_{\tau}}\left|g_{k, r}(t, x)\right| p\left(|x|-C_{0}(\tau-t)-R\right) d t d x \\
\leqslant & \int_{\mathbb{R}^{n}}\left(\left|u_{0}(x)\right|+4\right) p\left(|x|-C_{0} \tau-R\right) d x \\
& +\int_{\Pi_{\tau}}|g(t, x)| p\left(|x|-C_{0}(\tau-t)-R\right) d t d x=C(R, \tau) .
\end{aligned}
$$


По лемме Фату в пределе при $k \rightarrow \infty$ получаем

$$
\int_{\mathbb{R}^{n}}\left|u_{r}(\tau, x)\right| p(|x|-R) d x \leqslant C(R, \tau)
$$

для всех $R>0, r \in \mathbb{N}$ и п.в. $\tau \in(0, T)$. В частности, $u_{r}(t, x) \in L_{\mathrm{loc}}^{1}\left(\bar{\Pi}_{T}\right)$ и $u_{k, r} \rightarrow u_{r}$ в $L_{\mathrm{loc}}^{1}\left(\bar{\Pi}_{T}\right)$. Очевидно также, что при $k \rightarrow \infty$

$$
u_{k, r}^{0} \rightarrow u_{r}^{0}(x)=\left(u_{0}\right)^{+}-\left(u_{0}\right)^{-} \chi_{|x|<r} \quad \text { в } \quad L_{\text {loc }}^{1}\left(\mathbb{R}^{n}\right)
$$

и

$$
g_{k, r} \rightarrow g_{r}(t, x)=g^{+}-g^{-} \chi_{|x|<r} \quad \text { в } \quad L_{\mathrm{loc}}^{1}\left(\bar{\Pi}_{T}\right)
$$

По теореме $1 u_{r}(t, x)$ - о.э.p. задачи $(1),(2)$ с входными данными $\left(u_{r}^{0}, g_{r}\right)$. Итак, определена последовательность отображений $\mathscr{F}_{r}: L_{\mathrm{loc}}^{1}\left(\mathbb{R}^{n}\right) \times L_{\mathrm{loc}}^{1}\left(\bar{\Pi}_{T}\right) \mapsto L_{\mathrm{loc}}^{1}\left(\bar{\Pi}_{T}\right)$, $\mathscr{F}_{r}\left(u_{0}, g\right)=u_{r}(t, x)$. Далее, пусть $u_{0}, v_{0} \in L_{\mathrm{loc}}^{1}\left(\mathbb{R}^{n}\right), g, h \in L_{\mathrm{loc}}^{1}\left(\bar{\Pi}_{T}\right), u_{k, r}=$ $\mathscr{F}_{k, r}\left(u_{0}, g\right), v_{k, r}=\mathscr{F}_{k, r}\left(v_{0}, h\right)$. Из очевидных неравенств $\left(u^{+}-v^{+}\right)^{+} \leqslant(u-v)^{+}$, $\left(v^{-}-u^{-}\right)^{+} \leqslant(u-v)^{+}$легко вытекают оценки $\left(u_{k, r}^{0}-v_{k, r}^{0}\right)^{+} \leqslant\left(u_{0}-v_{0}\right)^{+}$, $\left(g_{k, r}-h_{k, r}\right)^{+} \leqslant(g-h)^{+}$. Далее, так как для отображения $\mathscr{F}$ выполнено свойство (36), то для любых $k, r \in \mathbb{N}$ при п.в. $\tau \in(0, T)$

$$
\begin{aligned}
\int_{\mathbb{R}^{n}}\left(u_{k, r}(\tau, x)-v_{k, r}(\tau, x)\right)^{+} d x \leqslant & \int_{\mathbb{R}^{n}}\left(u_{k, r}^{0}(x)-v_{k, r}^{0}(x)\right)^{+} d x \\
& +\int_{\Pi_{\tau}}\left[u_{k, r}-v_{k, r},\left(g_{k, r}-h_{k, r}\right)^{+}\right] d t d x \\
\leqslant & \int_{\mathbb{R}^{n}}\left(u_{0}(x)-v_{0}(x)\right)^{+} d x \\
& +\int_{\Pi_{\tau}}\left[u_{k, r}-v_{k, r},(g-h)^{+}\right]_{\beta} d t d x
\end{aligned}
$$

для всех $\beta>0$, откуда в пределе при $k \rightarrow \infty$ следует, что для п.в. $\tau \in(0, T)$

$\int_{\mathbb{R}^{n}}\left(u_{r}(\tau, x)-v_{r}(\tau, x)\right)^{+} d x \leqslant \int_{\mathbb{R}^{n}}\left(u_{0}(x)-v_{0}(x)\right)^{+} d x+\int_{\Pi_{\tau}}\left[u_{r}-v_{r},(g-h)^{+}\right]_{\beta} d t d x$,

где $u_{r}=\mathscr{F}_{r}\left(u_{0}, g\right), v_{r}=\mathscr{F}_{r}\left(v_{0}, h\right)$. Мы учли, что при $k \rightarrow \infty$

$$
\left[u_{k, r}-v_{k, r},(g-h)^{+}\right]_{\beta} \rightarrow\left[u_{r}-v_{r},(g-h)^{+}\right]_{\beta}
$$

и

$$
0 \leqslant\left[u_{k, r}-v_{k, r},(g-h)^{+}\right]_{\beta} \leqslant(g-h)^{+} \in L^{1}\left(\Pi_{T}\right),
$$

что позволяет применить теорему Лебега о предельном переходе под знаком интеграла.

По свойству монотонности (40) почти всюду на $\Pi_{T}$ определена предельная функция $u=u(t, x)=\lim _{r \rightarrow \infty} u_{r}(t, x) \geqslant-\infty$. Переходя к пределу при $r \rightarrow \infty$ в оценке (41), получим

$$
\int_{\mathbb{R}^{n}}|u(\tau, x)| p(|x|-R) d x \leqslant C(R, \tau)
$$

для всех $R>0$ и п.в. $\tau \in(0, T)$. Поэтому $u(t, x) \in L_{\text {loc }}^{1}\left(\bar{\Pi}_{T}\right)$ и $u_{r} \rightarrow u$ при $r \rightarrow \infty$ в $L_{\text {loc }}^{1}\left(\bar{\Pi}_{T}\right)$. Поскольку также $u_{r}^{0} \rightarrow u_{0}$ при $r \rightarrow \infty$ в $L_{\text {loc }}^{1}\left(\mathbb{R}^{n}\right)$ и $g_{r} \rightarrow g$ при $r \rightarrow \infty$ в $L_{\text {loc }}^{1}\left(\bar{\Pi}_{T}\right)$, то снова по теореме $1 u=u(t, x)-$ о.э.р. задачи $(1),(2)$ с входными данными $\left(u_{0}, g\right)$. Положим $\mathscr{F}\left(u_{0}, g\right)=u$. Переходя в неравенстве $(42) \mathrm{k}$ пределу сначала при $r \rightarrow \infty$, а затем при $\beta \rightarrow 0$, получим, что выполнено свойство (36). Теорема доказана. 
СлЕДСТВИЕ 3. 1) Если $u_{0} \leqslant v_{0}$ n.в. на $\mathbb{R}^{n}, g \leqslant h$ n.в. на $\Pi_{T} u u=\mathscr{F}\left(u_{0}, g\right)$, $v=\mathscr{F}\left(v_{0}, h\right)$, то $u \leqslant v$ п.в. на $\Pi_{T}$ (принцип сравнения).

2) Ecлu $u_{0}-v_{0} \in L^{1}\left(\mathbb{R}^{n}\right), \quad g-h \in L^{1}\left(\Pi_{T}\right) u u=\mathscr{F}\left(u_{0}, g\right), v=\mathscr{F}\left(v_{0}, h\right), m o$ для п.в. $\tau \in(0, T) \quad u(\tau, \cdot)-v(\tau, \cdot) \in L^{1}\left(\mathbb{R}^{n}\right) u$

$$
\begin{aligned}
\|u(\tau, \cdot)-v(\tau, \cdot)\|_{1} & \leqslant\left\|u_{0}-v_{0}\right\|_{1}+\int_{\Pi_{\tau}}\left[\chi_{u \geqslant v}(g-h)^{+}+\chi_{v \geqslant u}(h-g)^{+}\right] d t d x \\
& \leqslant\left\|u_{0}-v_{0}\right\|_{1}+\|g-h\|_{L^{1}\left(\Pi_{\tau}\right)}
\end{aligned}
$$

( устойчивость по $L^{1}$-норме).

ДокАЗАТЕльСТво. Первое утверждение непосредственно следует из (36). Для доказательства оценки 2) нужно поменять местами функции $u$ и $v$ в (36) и сложить полученное и исходное неравенства.

Рассмотрим теперь общий случай. Обозначим $S \subset L_{\text {loc }}^{1}\left(\bar{\Pi}_{T} \times \mathbb{R}\right)$ множество, состоящее из функций $g(t, x, u)$, удовлетворяюших условию (4) (с константой $L=$ $L(g)$, которая зависит от выбора $g(t, x, u))$. Справедлив следуюший технический результат об аппроксимации функций из $S$.

ЛЕмма 2. Пусть функиия $g=g(t, x, u) \in S$ ограничена снизу: $g(t, x, u) \geqslant$ $-M, u L=L(g)$,

$$
g_{k}(t, x, u)=\min _{v \in \mathbb{R}}\{g(t, x, v)+k|v-u|\} .
$$

Тогда $g_{k}(t, x, u) \in S ;-M \leqslant g_{k} \leqslant g_{k+1} \leqslant g, \quad k \in \mathbb{N} ; g_{k}(t, x, u) \rightarrow g(t, x, u)$ nри $k \rightarrow \infty ; g_{k}\left(t, x, u_{2}\right)-g_{k}\left(t, x, u_{1}\right) \leqslant L\left(u_{2}-u_{1}\right)$ при всех $u_{2}>u_{1}$ и выполнено условие Липчиц $\left|g_{k}\left(t, x, u_{2}\right)-g_{k}\left(t, x, u_{1}\right)\right| \leqslant k\left|u_{2}-u_{1}\right|, u_{1}, u_{2} \in \mathbb{R}$.

ДокАЗАТЕЛЬСтво. Заметим, что из ограниченности снизу функции $g$ следует сушествование минимума в равенстве, определяющем функции $g_{k}$. Ясно, что $g_{k}(t, x, u)=\inf _{v \in \mathbb{Q}}\{g(t, x, v)+k|v-u|\}$ и $g_{k}(t, x, u)$ измерима по $(t, x) \in \Pi_{T}$ и по совокупности переменных как точная нижняя грань счетного множества измеримых функций. Неравенства $-M \leqslant g_{k} \leqslant g_{k+1} \leqslant g, k \in \mathbb{N}$, сразу следуют из определения функций $g_{k}$. Пусть $g_{k}(t, x, u)=g\left(t, x, v_{k}\right)+k\left|u-v_{k}\right|, v_{k} \in \mathbb{R}$. Тогда

$$
k\left|u-v_{k}\right|=g_{k}(t, x, u)-g\left(t, x, v_{k}\right) \leqslant g(t, x, u)+M,
$$

откуда следует, что $v_{k} \rightarrow u$ при $k \rightarrow \infty$. Ввиду непрерывности $g(t, x, u)$ по переменной $u, g\left(t, x, v_{k}\right) \rightarrow g(t, x, u)$ при $k \rightarrow \infty$ и

$$
\liminf _{k \rightarrow \infty} g_{k}(t, x, u) \geqslant \lim _{k \rightarrow \infty} g\left(t, x, v_{k}\right)=g(t, x, u) .
$$

Учитывая также, что $g_{k}(t, x, u) \leqslant g(t, x, u)$, получаем требуемое предельное соотношение $g_{k}(t, x, u) \rightarrow g(t, x, u)$ при $k \rightarrow \infty$.

Покажем, что функции $g_{k}(t, x, u)$ удовлетворяют условию Липшица по $u$. Пусть $u_{1}, u_{2} \in \mathbb{R}$. Тогда

$$
\begin{aligned}
g_{k}\left(t, x, u_{1}\right) & =\min _{v}\left\{g(t, x, v)+k\left|v-u_{1}\right|\right\} \\
& \leqslant \min _{v}\left\{g(t, x, v)+k\left|v-u_{2}\right|\right\}+k\left|u_{1}-u_{2}\right| \\
& =g_{k}\left(t, x, u_{2}\right)+k\left|u_{1}-u_{2}\right|
\end{aligned}
$$


т.е. $g_{k}\left(t, x, u_{1}\right)-g_{k}\left(t, x, u_{2}\right) \leqslant k\left|u_{1}-u_{2}\right|$. Меняя местами $u_{1}$ и $u_{2}$ в этом неравенстве, получим требуемую оценку: $\left|g_{k}\left(t, x, u_{1}\right)-g_{k}\left(t, x, u_{2}\right)\right| \leqslant k\left|u_{1}-u_{2}\right|$.

Пусть, далее, $u_{2}>u_{1}$. Сделав замену $h=v-u_{2}$, получим с учетом условия (4), что

$$
\begin{aligned}
g_{k}\left(t, x, u_{2}\right) & =\min _{h}\left\{g\left(t, x, u_{2}+h\right)+k|h|\right\} \\
& \leqslant \min _{h}\left\{g\left(t, x, u_{1}+h\right)+k|h|\right\}+L\left(u_{2}-u_{1}\right) \\
& =g_{k}\left(t, x, u_{1}\right)+L\left(u_{2}-u_{1}\right) .
\end{aligned}
$$

Итак, $g_{k}\left(t, x, u_{2}\right)-g_{k}\left(t, x, u_{1}\right) \leqslant L\left(u_{2}-u_{1}\right)$. Лемма доказана.

Tеорема 3. Существует отображсение $\mathscr{F}: L_{\mathrm{loc}}^{1}\left(\mathbb{R}^{n}\right) \times S \mapsto L_{\mathrm{loc}}^{1}\left(\bar{\Pi}_{T}\right)$, conoставляющее входным данным $\left(u_{0}, g\right)$ задачи (1), (2) о.ә.р. $u=\mathscr{F}\left(u_{0}, g\right)$ maк, что выполнено следующее свойство монотонной зависимости от входных данных: если $u=\mathscr{F}\left(u_{0}, g\right), \quad v=\mathscr{F}\left(v_{0}, h\right) u u_{0} \leqslant v_{0}$ n.в. на $\mathbb{R}^{n}, g \leqslant h$ n.в. на $\Pi_{T} \times \mathbb{R}$, то $u \leqslant v$ n.в. на $\Pi_{T}$.

ДокАЗАТЕЛЬСтво. Рассмотрим последовательно четыре случая, в каждом из которых отображение $\mathscr{F}$ строится для специальных классов функций $g(t, x, u)$.

А) Пусть функция $g(t, x, u) \in S$ удовлетворяет условию Липшица по последней переменной: $|g(t, x, u)-g(t, x, v)| \leqslant L|u-v| \forall u, v \in \mathbb{R}, L=$ const и сушествует $R>0$ такое, что $g(t, x, u)=-M=$ const при $|x|>R$. Докажем сушествование единственного о.э.p. $u=u(t, x)$ задачи $(1),(2)$ такого, что $u=\mathscr{F}\left(u_{0}, \bar{g}\right), \quad \bar{g}=$ $g(t, x, u(t, x)), \mathscr{F}$ - отображение из теоремы 2. Будем искать это о.э.р. методом последовательных приближений. Положим при $\bar{g}_{0}=g(t, x, 0)$

$$
u_{1}=\mathscr{F}\left(u_{0}, \bar{g}_{0}\right), \quad u_{k+1}=\mathscr{F}\left(u_{0}, \bar{g}_{k}\right), \quad \bar{g}_{k}(t, x)=g\left(t, x, u_{k}(t, x)\right), \quad k \in \mathbb{N} .
$$

Покажем, что для всех $k \in \mathbb{N}$

$$
\begin{aligned}
u_{k} & \in L_{\text {loc }}^{1}\left(\bar{\Pi}_{T}\right), \\
u_{k+1}(\tau, \cdot)-u_{k}(\tau, \cdot) & \in L^{1}\left(\mathbb{R}^{n}\right) \text { для п.в. } \tau \in(0, T), \\
\left\|u_{k+1}(\tau, \cdot)-u_{k}(\tau, \cdot)\right\|_{1} & \leqslant C(L \tau)^{k-1} /(k-1) !,
\end{aligned}
$$

где $C=L\left\|u_{1} \chi_{|x| \leqslant R}\right\|_{L^{1}\left(\Pi_{T}\right)}$. По построению $u_{1} \in L_{\text {loc }}^{1}\left(\bar{\Pi}_{T}\right)$, откуда следует, что $g_{1} \in L_{\mathrm{loc}}^{1}\left(\bar{\Pi}_{T}\right)$ и, значит, определено $u_{2} \in L_{\mathrm{loc}}^{1}\left(\bar{\Pi}_{T}\right)$. По следствию 3,2$)$ для п.в. $\tau \in(0, T)$

$$
\left\|u_{2}(\tau, \cdot)-u_{1}(\tau, \cdot)\right\|_{1} \leqslant T\left\|\bar{g}_{1}-\bar{g}_{0}\right\|_{L^{1}\left(\Pi_{\tau}\right)} \leqslant L\left\|u_{1} \chi_{|x| \leqslant R}\right\|_{L^{1}\left(\Pi_{T}\right)}=C .
$$

Итак, условие (44) выполнено при $k=1$. В соответствии с методом математической индукции предположим, что (44) верно при некотором $k=r-1 \in \mathbb{N}$, и покажем, что оно вьполнено и при $k=r$. Поскольку $u_{r-1} \in L_{\text {loc }}^{1}\left(\bar{\Pi}_{T}\right), u_{r}-$ $u_{r-1} \in L^{1}\left(\Pi_{T}\right)$, то $u_{r} \in L_{\text {loc }}^{1}\left(\bar{\Pi}_{T}\right)$. Следовательно, $\bar{g}_{r} \in L_{\text {loc }}^{1}\left(\bar{\Pi}_{T}\right)$ и определено $u_{r+1}=\mathscr{F}\left(u_{0}, \bar{g}_{r}\right) \in L_{\text {loc }}^{1}\left(\bar{\Pi}_{T}\right)$. По следствию 3,2$)$ для п.в. $\tau \in(0, T)$

$$
\begin{aligned}
\left\|u_{r+1}(\tau, \cdot)-u_{r}(\tau, \cdot)\right\|_{L^{1}\left(\mathbb{R}^{n}\right)} & \leqslant\left\|\bar{g}_{r}-\bar{g}_{r-1}\right\|_{L^{1}\left(\Pi_{\tau}\right)} \leqslant L\left\|u_{r}-u_{r-1}\right\|_{L^{1}\left(\Pi_{\tau}\right)} \\
& =L \int_{0}^{\tau}\left\|u_{r}(t, \cdot)-u_{r-1}(t, \cdot)\right\|_{1} d t \\
& \leqslant L \int_{0}^{\tau} C L(L t)^{r-2} /(r-2) ! d t \\
& =C(L \tau)^{r-1} /(r-1) !
\end{aligned}
$$


В последнем неравенстве мы воспользовались индукционным предположением. По принципу математической индукции условие (44) верно для всех $k \in \mathbb{N}$. Из (44) после интегрирования по $\tau \in(0, T)$ следует оценка

$$
\left\|u_{k+1}-u_{k}\right\|_{L^{1}\left(\Pi_{T}\right)} \leqslant \frac{C}{L} \frac{(L T)^{k}}{k !} .
$$

Ввиду сходимости ряда $\sum_{k=1}^{\infty}(L T)^{k} / k !$ последовательность $u_{k}-u_{1}$ фундаментальна в $L^{1}\left(\Pi_{T}\right)$, откуда вытекает ее сходимость в $L^{1}\left(\Pi_{T}\right)$. В частности, последовательность $u_{k}$ сходится в $L_{\mathrm{loc}}^{1}\left(\bar{\Pi}_{T}\right)$ к некоторой функции $u=u(t, x) \in L_{\mathrm{loc}}^{1}\left(\bar{\Pi}_{T}\right)$. Пусть $\bar{g}(t, x)=g(t, x, u(t, x)), v=v(t, x)=\mathscr{F}\left(u_{0}, \bar{g}\right)$. Тогда

$$
\left|\bar{g}_{k}(t, x)-\bar{g}\right| \leqslant L\left|u_{k}-u\right| \chi_{|x| \leqslant R}
$$

откуда следует, что $\bar{g}_{k}(t, x)-\bar{g} \rightarrow 0$ в $L^{1}\left(\Pi_{T}\right)$. По (43) и следствию 3,2$)$

$$
\left\|u_{k+1}-v\right\|_{L^{1}\left(\Pi_{T}\right)} \leqslant T\left\|\bar{g}_{k}-\bar{g}\right\|_{L^{1}\left(\Pi_{T}\right)} \underset{k \rightarrow \infty}{\longrightarrow} 0
$$

и $u_{k} \rightarrow v$ в $L_{\text {loc }}^{1}\left(\bar{\Pi}_{T}\right)$. Поэтому $u=v$ п.в. на $\Pi_{T}$ и $u=v=\mathscr{F}\left(u_{0}, \bar{g}\right), \bar{g}=$ $g(t, x, u(t, x))$. В частности, $u-$ o.э.р. задачи $(1),(2)$. Для доказательства свойства монотонности предположим, что $u=\mathscr{F}\left(u_{0}, \bar{g}\right), v=\mathscr{F}\left(v_{0}, \bar{h}\right)$, где $u_{0} \leqslant v_{0}$ п.в. на $\mathbb{R}^{n}$, $g \leqslant h$ п.в. на $\Pi_{T} \times \mathbb{R}$ и $\bar{g}(t, x)=g(t, x, u(t, x)), \bar{h}(t, x)=h(t, x, u(t, x))$. Выберем величины $L, R$ обшими для функций $g$ и $h$. Ввиду (36) для п.в. $\tau \in(0, T)$

$$
\begin{aligned}
& \int_{|x|<R}(u(\tau, x)-v(\tau, x))^{+} d x \leqslant \int_{\mathbb{R}^{n}}(u(\tau, x)-v(\tau, x))^{+} d x \\
& \leqslant \int_{0}^{\tau} \int_{|x|<R} \chi_{u \geqslant v}(g(t, x, u(t, x))-h(t, x, v(t, x)))^{+} d x d t \\
& \leqslant \int_{0}^{\tau} \int_{|x|<R}\left[\chi_{u \geqslant v}(g(t, x, u(t, x))-g(t, x, v(t, x)))^{+}\right. \\
&\left.+(g(t, x, v)-h(t, x, v))^{+}\right] d x d t \\
& \leqslant L \int_{0}^{\tau} \int_{|x|<R}(u(t, x)-v(t, x))^{+} d x d t .
\end{aligned}
$$

Используя лемму Гронуолла, получаем, что $\int_{|x|<R}(u(\tau, x)-v(\tau, x))^{+} d x=0$ для п.в. $\tau \in(0, T)$, откуда

$$
\int_{\mathbb{R}^{n}}(u(\tau, x)-v(\tau, x))^{+} d x \leqslant L \int_{0}^{\tau} \int_{|x|<R}(u(t, x)-v(t, x))^{+} d x d t=0
$$

для п.в. $\tau \in(0, T)$, т.е. $u(t, x) \leqslant v(t, x)$ п.в. на $\Pi_{T}$. Ясно, что из установленного свойства монотонности следует единственность функции $u(t, x)$ такой, что $u=\mathscr{F}\left(u_{0}, \bar{g}\right), \bar{g}=g(t, x, u(t, x))$.

В) Пусть функция $g(t, x, u) \in S$ ограничена снизу и удовлетворяет условию Липшица $|g(t, x, u)-g(t, x, v)| \leqslant L|u-v| \forall u, v \in \mathbb{R}, L=$ const.

Выберем $M$ так, что $g(t, x, u) \geqslant-M$ п.в. на $\Pi_{T} \times \mathbb{R}$, и положим при $k, r \in \mathbb{N}$, $k>M$,

$$
g_{k, r}(t, x, u)= \begin{cases}g(t, x, u), & |x| \leqslant r \\ -k, & |x|>r .\end{cases}
$$


Как установлено в п. А), существует о.э.p. $u_{k, r}(t, x)$ задачи Коши $(1),(2)$ с входными данными $\left(u_{0}, g_{k, r}\right)$, удовлетворяющее свойству монотонности. Очевидно, $g_{k+1, r} \leqslant g_{k, r} \leqslant g_{k, r+1}$ п.в. на $\Pi_{T} \times \mathbb{R}$ и по свойству монотонности $u_{k+1, r} \leqslant u_{k, r} \leqslant$ $u_{k, r+1}$ п.в. на $\Pi_{T}$. В частности, для п.в. $(t, x) \in \Pi_{T}$ определены предельные функции $u_{k}(t, x)=\lim _{r \rightarrow \infty} u_{k, r}(t, x) \leqslant+\infty$ и $u_{k+1}(t, x) \leqslant u_{k}(t, x)$ п.в. на П $\Pi_{T}$. По следствию 2 для всех $R>0$ и п.в. $\tau \in(0, T)$ имеем

$$
\begin{aligned}
\int_{\mathbb{R}^{n}}\left|u_{k, r}(\tau, x)\right| p(|x|-R) d x \leqslant & e^{L \tau}\left(\int_{\mathbb{R}^{n}}\left(\left|u_{0}(x)\right|+4\right) p\left(|x|-C_{0} \tau-R\right) d x\right. \\
& \left.+\int_{\Pi_{\tau}}\left|g_{k, r}(t, x, 0)\right| p\left(|x|-C_{0}(\tau-t)-R\right) d t d x\right),
\end{aligned}
$$

откуда в пределе при $r \rightarrow \infty$ получим с использованием леммы Фату, что для всех $R>0$ и п.в. $\tau \in(0, T)$

$$
\begin{aligned}
\int_{\mathbb{R}^{n}}\left|u_{k}(\tau, x)\right| p(|x|-R) d x \leqslant & C(\tau, R)=e^{L \tau}\left(\int_{\mathbb{R}^{n}}\left(\left|u_{0}(x)\right|+4\right) p\left(|x|-C_{0} \tau-R\right) d x\right. \\
& \left.+\int_{\Pi_{\tau}}|g(t, x, 0)| p\left(|x|-C_{0}(\tau-t)-R\right) d t d x\right)
\end{aligned}
$$

Поэтому $u_{k}(t, x) \in L_{\text {lос }}^{1}\left(\bar{\Pi}_{T}\right)$ и $u_{k, r} \rightarrow u_{k}$ при $r \rightarrow \infty$ в $L_{\text {lос }}^{1}\left(\bar{\Pi}_{T}\right)$. Поскольку $g_{k, r}(t, x, u)=g(t, x, u)$ при $|x| \leqslant r$, то $g_{k, r}(t, x, u) \rightarrow g(t, x, u)$ при $r \rightarrow \infty$ для любого $u \in \mathbb{R}$ в $L_{\text {loc }}^{1}\left(\bar{\Pi}_{T}\right)$. Тогда по теореме $1 u_{k}-$ о.э.р. задачи $(1),(2)$ с входными данными $\left(u_{0}, g\right)$ для всех $k \in \mathbb{N}$. Из монотонности последовательности $u_{k}(t, x)$ следует, что при $k \rightarrow \infty u_{k}(t, x) \rightarrow u(t, x)$ п.в. на $\Pi_{T}$, причем, как следует из (45) в пределе при $k \rightarrow \infty$, для всех $R>0$ и п.в. $\tau \in(0, T)$

$$
\int_{\mathbb{R}^{n}}|u(\tau, x)| p(|x|-R) d x \leqslant C(\tau, R) .
$$

Поэтому $u(t, x) \in L_{\mathrm{loc}}^{1}\left(\bar{\Pi}_{T}\right)$ и $u_{k} \rightarrow u$ при $k \rightarrow \infty$ в $L_{\mathrm{loc}}^{1}\left(\bar{\Pi}_{T}\right)$. Снова используя теорему 1 , заключаем, что $u(t, x)$ - о.э.р. задачи $(1),(2)$ с входными данными $\left(u_{0}, g\right)$. Положим $u=\mathscr{F}\left(u_{0}, g\right)$. Пусть $v=\mathscr{F}\left(v_{0}, h\right)$, где $u_{0} \leqslant v_{0}$ п.в. на $\mathbb{R}^{n}, g \leqslant h$ п.в. на $\Pi_{T} \times \mathbb{R}$. Тогда при достаточно больших $k \in \mathbb{N}$ и всех $r \in \mathbb{N}$ $g_{k, r}(t, x, u) \leqslant h_{k, r}(t, x, u)$ и по свойству монотонности для случая A) $u_{k, r} \leqslant v_{k, r}$ п.в. на $\Pi_{T}$, где $u_{k, r}=\mathscr{F}\left(u_{0}, g_{k, r}\right), v_{k, r}=\mathscr{F}\left(v_{0}, h_{k, r}\right)$. В пределе, сначала при $r \rightarrow \infty$, а затем при $k \rightarrow \infty$, получаем, что $u \leqslant v$ п.в. на $\Pi_{T}$, т.е. выполнено свойство монотонной зависимости от входных данных.

C) Пусть функция $g(t, x, u) \in S$ ограничена снизу: $g \geqslant-M$. Положим $L=L(g)$, $g_{k}(t, x, u)=\min _{v \in \mathbb{R}}\{g(t, x, v)+k|v-u|\}$.

По лемме 2 имеем $-M \leqslant g_{k} \leqslant g_{k+1} \leqslant g, k \in \mathbb{N}, g_{k}(t, x, u) \rightarrow g(t, x, u)$ при $k \rightarrow \infty$, причем $g_{k}\left(t, x, u_{2}\right)-g_{k}\left(t, x, u_{1}\right) \leqslant L\left(u_{2}-u_{1}\right)$ при всех $u_{2}>u_{1}$ и функции $g_{k}$ удовлетворяют условию Липшица по последней переменной: $\mid g_{k}\left(t, x, u_{2}\right)-$ $g_{k}\left(t, x, u_{1}\right)|\leqslant k| u_{2}-u_{1} \mid, u_{1}, u_{2} \in \mathbb{R}$.

Функции $g_{k}$ удовлетворяют условию п. В) и, значит, определены о.э.р. $u_{k}=$ $\mathscr{F}\left(u_{0}, g_{k}\right) \in L_{\text {lос }}^{1}\left(\bar{\Pi}_{T}\right)$, причем по свойству монотонности $u_{k} \leqslant u_{k+1}$ п.в. на $\Pi_{T}$ для всех $k \in \mathbb{N}$. Поэтому для п.в. $(t, x) \in \Pi_{T}$ определена предельная функция $u(t, x)=$ 
$\lim _{k \rightarrow \infty} u_{k}(t, x)$. По следствию 2, с учетом оценки $\left|g_{k}(t, x, 0)\right| \leqslant g_{k}(t, x, 0)+2 M \leqslant$ $g(t, x, 0)+2 M$, для всех $R>0$ и п.в. $\tau \in(0, T)$

$$
\begin{array}{r}
\int_{\mathbb{R}^{n}}\left|u_{k}(\tau, x)\right| p(|x|-R) d x \leqslant \\
C_{1}(\tau, R)=e^{L \tau}\left(\int_{\mathbb{R}^{n}}\left(\left|u_{0}(x)\right|+4\right) p\left(|x|-C_{0} \tau-R\right) d x\right. \\
\left.+\int_{\Pi_{\tau}}(g(t, x, 0)+2 M) p\left(|x|-C_{0}(\tau-t)-R\right) d t d x\right),
\end{array}
$$

откуда в пределе при $k \rightarrow \infty$ получим (по лемме Фату), что для всех $R>0$ и п.в. $\tau \in(0, T)$

$$
\int_{\mathbb{R}^{n}}|u(\tau, x)| p(|x|-R) d x \leqslant C_{1}(\tau, R) .
$$

Поэтому $u(t, x) \in L_{\text {loc }}^{1}\left(\bar{\Pi}_{T}\right)$ и $u_{k} \rightarrow u$ при $k \rightarrow \infty$ в $L_{\text {loc }}^{1}\left(\bar{\Pi}_{T}\right)$. По построению $g_{k}(t, x, u) \rightarrow g(t, x, u)$ при $k \rightarrow \infty$ для всех $(t, x, u)$. Учитывая оценки $-M \leqslant$ $g_{k}(t, x, u) \leqslant g(t, x, u) \in L_{\text {lос }}^{1}\left(\bar{\Pi}_{T}\right)$, получаем, что $g_{k}(t, x, u) \rightarrow g(t, x, u)$ при $k \rightarrow \infty$ в $L_{\text {loc }}^{1}\left(\bar{\Pi}_{T}\right)$ для всех $u \in \mathbb{R}$. Тогда из теоремы 1 следует, что $u(t, x)-$ о.э.р. задачи $(1),(2)$ с входными данными $\left(u_{0}, g\right)$. Положим $u=\mathscr{F}\left(u_{0}, g\right)$.

Для доказательства свойства монотонности положим, что $u_{0}, v_{0} \in L_{\text {loc }}^{1}\left(\mathbb{R}^{n}\right)$, функции $g, h \in S$ ограничены снизу и $u_{0} \leqslant v_{0}$ п.в. на $\mathbb{R}^{n}, g \leqslant h$ п.в. на $\Pi_{T} \times \mathbb{R}$. Пусть $u=\mathscr{F}\left(u_{0}, g\right), v=\mathscr{F}\left(v_{0}, h\right)$. Пусть

$$
g_{k}(t, x, u)=\min _{v \in \mathbb{R}}\{g(t, x, v)+k|v-u|\}, \quad h_{k}(t, x, u)=\min _{v \in \mathbb{R}}\{h(t, x, v)+k|v-u|\} .
$$

Тогда, очевидно, $g_{k}(t, x, u) \leqslant h_{k}(t, x, u)$ п.в. на $\Pi_{T} \times \mathbb{R}$ и по свойству монотонности для случая В) $u_{k}=\mathscr{F}\left(u_{0}, g_{k}\right) \leqslant v_{k}=\mathscr{F}\left(v_{0}, h_{k}\right)$ п.в. на $\Pi_{T}$. В пределе $u_{k} \rightarrow u$, $v_{k} \rightarrow v$ при $k \rightarrow \infty$ п.в. на $\Pi_{T}$ и, следовательно, $u \leqslant v$ п.в. на $\Pi_{T}$.

D) Обший случай: $g \in S$. Положим $g_{k}=g_{k}(t, x, u)=\max (g(t, x, u),-k)$ при $k \in \mathbb{N}$. Тогда функции $g_{k}$ ограничены снизу и определены о.э.р. $u_{k}=\mathscr{F}\left(u_{0}, g_{k}\right)$, где $\mathscr{F}$ - отображение, построенное в п. С). Так как $g_{k+1}(t, x, u) \leqslant g_{k}(t, x, u), k \in \mathbb{N}$, то по свойству монотонности $u_{k+1}(t, x) \leqslant u_{k}(t, x)$ п.в. на $\Pi_{T}$ и для п.в. $(t, x) \in \Pi_{T}$ определена предельная функция $u(t, x)=\lim _{k \rightarrow \infty} u_{k}(t, x)$. Применяя следствие 2 к о.э.p. $u_{k}(t, x)$ и переходя к пределу при $k \rightarrow \infty$, получим оценку: для всех $R>0$ и п.в. $\tau \in(0, T)$

$$
\int_{\mathbb{R}^{n}}|u(\tau, x)| p(|x|-R) d x \leqslant C(\tau, R),
$$

из которой следует, что $u(t, x) \in L_{\mathrm{loc}}^{1}\left(\bar{\Pi}_{T}\right)$ и $u_{k} \rightarrow u$ при $k \rightarrow \infty$ в $L_{\mathrm{loc}}^{1}\left(\bar{\Pi}_{T}\right)$. Очевидно также, что $g_{k}(t, x, u) \rightarrow g(t, x, u)$ в $L_{\text {loc }}^{1}\left(\bar{\Pi}_{T}\right) \quad \forall u \in \mathbb{R}$ и $L\left(g_{k}\right)=L(g)$, $k \in \mathbb{N}$. По теореме $1 u=u(t, x)-$ o.э.р. задачи $(1),(2)$ с входными данными $\left(u_{0}, g\right)$. Положим $u=\mathscr{F}\left(u_{0}, g\right)$.

Пусть $u_{0}, v_{0} \in L_{\text {loc }}^{1}\left(\mathbb{R}^{n}\right), g, h \in S ; u_{0} \leqslant v_{0}$ п.в. на $\mathbb{R}^{n}, g \leqslant h$ п.в. на $\Pi_{T} \times \mathbb{R}$ и $u=\mathscr{F}\left(u_{0}, g\right), v=\mathscr{F}\left(v_{0}, h\right)$. Тогда п.в. на $\Pi_{T} u_{k} \rightarrow u, v_{k} \rightarrow v$, где $u_{k}=\mathscr{F}\left(u_{0}, g_{k}\right)$, $v_{k}=\mathscr{F}\left(v_{0}, h_{k}\right), g_{k}=\max (g,-k), h_{k}=\max (h,-k)$. Ясно, что $g_{k} \leqslant h_{k}$ п.в. на $\Pi_{T} \times \mathbb{R}$ и по свойству монотонности для случая $\left.\mathrm{C}\right) u_{k} \leqslant v_{k}$ п.в. на $\Pi_{T}$, откуда в пределе при $k \rightarrow \infty$ выводим, что $u \leqslant v$ п.в. на $\Pi_{T}$. Теорема полностью доказана. 


\section{§ 3. О сушествовании наибольшего и наименьшего о.э.p.}

В рассматриваемом случае, когда функции потока лишь непрерывны, единственность о.э.р. задачи (1), (2) может нарушаться даже в классе ограниченных о.э.р. (см. примеры в [14], [15]). В этой связи важную роль играет результат о сушествовании наибольшего и наименьшего о.э.р., доказываемый в этом параграфе. Так, на базе этого результата в $\S 5$ будет установлена единственность о.э.р. в случае периодических входных данных задачи. В классе ограниченных о.э.р. существование наибольшего и наименьшего о.э.р. было установлено ранее, при различных дополнительных предположениях, в работах [5], [6], [1], [20].

Используемьй нами подход следует работе [19] и близок к известному методу Пуанкаре-Перрона доказательства разрешимости задачи Дирихле для уравнения Лапласа. Сначала мы устанавливаем, что точная верхняя грань любого множества о.э.субр. (точная нижняя грань множества о.э.суперр.) также является о.э.субр. (соответственно, о.э.суперр.) нашей задачи (отметим, что этот результат был доказан в классе ограниченных о.э.субр. и о.э.суперр. в [2], при некоторых дополнительных ограничениях на вектор потока). Таким образом, существуют наибольшее о.э.субр. и наименьшее о.э.суперр. задачи (1), (2) и остается лишь установить, что они являются, на самом деле, о.э.р. нашей задачи.

Нам понадобится следуюшая простая техническая

Лемма 3. Пусть $\Omega$ - открытое подмножество в $\mathbb{R}^{N} ; l \in \mathscr{D}^{\prime}(\Omega), \quad g, h \in$ $L_{\text {loc }}^{1}(\Omega) u l \leqslant g, \quad l \leqslant h$ в $\mathscr{D}^{\prime}(\Omega)$. Тогда $l \leqslant \min (g, h)$ в $\mathscr{D}^{\prime}(\Omega)$.

ДоказАТЕЛЬСТво. Фиксируем пробную функцию $f \in C_{0}^{\infty}(\Omega), f \geqslant 0$, и пусть $\chi \in C_{0}^{\infty}(\Omega), \quad \chi \geqslant 0, \quad \chi=1$ на $\operatorname{supp} f$. Рассмотрим распределение $\bar{l} \in \mathscr{D}^{\prime}\left(\mathbb{R}^{N}\right)$, действуюшее по правилу: $\langle\bar{l}, p\rangle=\langle l, \chi p\rangle, p \in C_{0}^{\infty}\left(\mathbb{R}^{N}\right)$. Ясно, что носитель распределения $\bar{l}$ содержится в $\operatorname{supp} \chi$ и $\bar{l} \leqslant \bar{g}, \bar{l} \leqslant \bar{h}$ в $\mathscr{D}^{\prime}\left(\mathbb{R}^{N}\right)$, где функции $\bar{g}, \bar{h} \in$ $L_{\mathrm{loc}}^{1}\left(\mathbb{R}^{N}\right)$ определяются равенствами $\bar{g}(x)=g(x) \chi(x), \bar{h}(x)=h(x) \chi(x)$ при $x \in \Omega$, $\bar{g}(x)=\bar{h}(x)=0$ при $x \notin \Omega$.

Пусть, далее, $\rho(x) \in C_{0}^{\infty}\left(\mathbb{R}^{N}\right), \rho(x) \geqslant 0, \int \rho(x) d x=1$. Положим $\rho_{\nu}(x)=$ $\nu^{N} \rho(\nu x)$ при $\nu \in \mathbb{N}$. Ясно, что последовательность $\rho_{\nu}(x)$ сходится к $\delta$-функции Дирака в пространстве $\mathscr{D}^{\prime}\left(\mathbb{R}^{N}\right)$. Рассмотрим свертки

$$
\begin{aligned}
& \bar{l}_{\nu}(x)=\left(\bar{l} * \rho_{\nu}\right)(x)=\left\langle\bar{l}(y), \rho_{\nu}(x-y)\right\rangle, \\
& \bar{g}_{\nu}(x)=\left(\bar{g} * \rho_{\nu}\right)(x)=\int \bar{g}(y) \rho_{\nu}(x-y) d y, \\
& \bar{h}_{\nu}(x)=\left(\bar{h} * \rho_{\nu}\right)(x)=\int \bar{h}(y) \rho_{\nu}(x-y) d y .
\end{aligned}
$$

Тогда $\bar{l}_{\nu}, \bar{g}_{\nu}, \bar{h}_{\nu} \in C_{0}^{\infty}\left(\mathbb{R}^{N}\right)$ и, как следует из неравенств $\bar{l} \leqslant \bar{g}, \bar{l} \leqslant \bar{h}$ в $\mathscr{D}^{\prime}\left(\mathbb{R}^{N}\right)$, $\bar{l}_{\nu} \leqslant \bar{g}_{\nu}, \bar{l}_{\nu} \leqslant \bar{h}_{\nu}$ поточечно на $\mathbb{R}^{N}$. Поэтому

$$
\bar{l}_{\nu} \leqslant \min \left(\bar{g}_{\nu}, \bar{h}_{\nu}\right) \text { на } \mathbb{R}^{N} .
$$

Хорошо известно (см., например, [8]), что при $\nu \rightarrow \infty$ последовательность $\bar{l}_{\nu} \rightarrow \bar{l}$ слабо в $\mathscr{D}^{\prime}\left(\mathbb{R}^{N}\right)$, а $\bar{g}_{\nu} \rightarrow \bar{g}, \bar{h}_{\nu} \rightarrow \bar{h}$ в $L_{\text {loc }}^{1}\left(\mathbb{R}^{N}\right)$ и из (46) в пределе при $\nu \rightarrow \infty$ следует, что $\bar{l} \leqslant \min (\bar{g}, \bar{h})$ в $\mathscr{D}^{\prime}\left(\mathbb{R}^{N}\right)$. Поэтому

$$
\langle l, f\rangle=\langle l, f \chi\rangle=\langle\bar{l}, f\rangle \leqslant\langle\min (\bar{g}, \bar{h}), f\rangle=\langle\min (g, h), f \chi\rangle=\langle\min (g, h), f\rangle,
$$


и, ввиду произвольности сделанного выбора пробной функции $f, l \leqslant \min (g, h)$ в $\mathscr{D}^{\prime}(\Omega)$. Лемма доказана.

Справедлива также следуюшая

ЛЕмма 4. Пусть $u=u(t, x)-$ о.ә.субр. задачи (1), (2). Тогда для любого $k \in \mathbb{R}$ и любой пробной функиии $f=f(t, x) \in C_{0}^{\infty}\left(\bar{\Pi}_{T}\right), f \geqslant 0$,

$$
\begin{gathered}
\int_{\Pi_{T}}\left\{(u-k)^{+} f_{t}+\operatorname{sign}^{+}(u-k)\left[\left(\varphi(u)-\varphi(k), \nabla_{x} f\right)+g f\right]-\chi_{u=k} g^{-} f\right\} d t d x \\
+\int_{\mathbb{R}^{n}}\left(u_{0}(x)-k\right)^{+} f(0, x) d x \geqslant 0 .
\end{gathered}
$$

В частности,

$$
\begin{aligned}
& \left((u-k)^{+}\right)_{t}+\operatorname{div}_{x}\left[\operatorname{sign}^{+}(u-k)(\varphi(u)-\varphi(k))\right] \\
& \quad \leqslant \operatorname{sign}^{+}(u-k) g-\chi_{u=k} g^{-} \quad \boldsymbol{B} \quad \mathscr{D}^{\prime}\left(\Pi_{T}\right) .
\end{aligned}
$$

ДокаЗАТЕЛЬСТво. Фиксируем $k \in \mathbb{R}$, и пусть $r<k, f=f(t, x) \in C_{0}^{\infty}\left(\Pi_{T}\right)$, $f \geqslant 0$. По соотношению $(20)$ имеем

$$
\int_{\Pi_{T}}\left\{(u-r)^{+} f_{t}+\operatorname{sign}^{+}(u-r)\left[\left(\varphi(u)-\varphi(r), \nabla_{x} f\right)+g f\right]\right\} d t d x \geqslant 0 .
$$

Поскольку при $r \rightarrow k, r<k, \operatorname{sign}^{+}(u-r) \rightarrow \operatorname{sign}^{+}(u-k)+\chi_{u=k}$, то из (49) в пределе при $r \rightarrow k$ вытекает неравенство: $\forall f=f(t, x) \in C_{0}^{\infty}\left(\Pi_{T}\right), f \geqslant 0$,

$$
\int_{\Pi_{T}}\left\{(u-k)^{+} f_{t}+\operatorname{sign}^{+}(u-k)\left[\left(\varphi(u)-\varphi(k), \nabla_{x} f\right)+g f\right]+\chi_{u=k} g f\right\} d t d x \geqslant 0,
$$

то есть

$$
\begin{array}{r}
\left((u-k)^{+}\right)_{t}+\operatorname{div}_{x}\left[\operatorname{sign}^{+}(u-k)(\varphi(u)-\varphi(k))\right] \\
\leqslant \operatorname{sign}^{+}(u-k) g+\chi_{u=k} g \quad \text { в } \mathscr{D}^{\prime}\left(\Pi_{T}\right) .
\end{array}
$$

По соотношению (20) также имеем

$$
\begin{gathered}
\left((u-k)^{+}\right)_{t}+\operatorname{div}_{x}\left[\operatorname{sign}^{+}(u-k)(\varphi(u)-\varphi(k))\right] \\
\leqslant \operatorname{sign}^{+}(u-k) g \text { в } \mathscr{D}^{\prime}\left(\Pi_{T}\right),
\end{gathered}
$$

и неравенство (48) следует из леммы 3 с учетом равенства

$$
\min \left(\operatorname{sign}^{+}(u-k) g+\chi_{u=k} g, \operatorname{sign}^{+}(u-k) g\right)=\operatorname{sign}^{+}(u-k) g-\chi_{u=k} g^{-} .
$$

В свою очередь, неравенство (47) выводится из (48) так же, как в первой части доказательства предложения 1 , с помошю предельного перехода, сначала при $\nu \rightarrow \infty$, а затем при $t_{0} \rightarrow 0$, в интегральном неравенстве, соответствуюшем (48) с пробной функцией $f_{\nu}(t, x)=f(t, x) \theta_{\nu}\left(t-t_{0}\right)$. Лемма доказана. 
Tеорема 4. 1) Если $u_{1}, u_{2}$ - о.ә.субр. задачи (1), (2), то $u=\max \left(u_{1}, u_{2}\right)$ яв.яется о.ә.субр. этой задачи.

2) Если $u_{1}, u_{2}$ - о.э.суперр. задачи (1), (2), то $u=\min \left(u_{1}, u_{2}\right)$ является о.ә.суперр. этой задачи.

ДокАЗАТЕльСтво. Ввиду замечания $2, \mathrm{~b})$, функция $u=u(t, x)-$ о.э.суперр. задачи (1), (2) тогда и только тогда, когда $-u$ является о.э.субр. задачи

$$
u_{t}-\operatorname{div}_{x} \varphi(-u)=-g(t, x,-u), \quad u(0, x)=-u_{0}(x) .
$$

Поэтому второе утверждение теоремы следует из первого.

Пусть $u=u(t, x)$ - о.э.субр. задачи (1), (2). По неравенству (48) для любого $k \in \mathbb{R}$

$$
\begin{aligned}
\max (u & , k)_{t}+\operatorname{div}_{x} \varphi(\max (u, k))= \\
& =\left((u-k)^{+}+k\right)_{t}+\operatorname{div}_{x}\left(\operatorname{sign}^{+}(u-k)(\varphi(u)-\varphi(k))+\varphi(k)\right) \\
& =\left((u-k)^{+}\right)_{t}+\operatorname{div}_{x}\left[\operatorname{sign}^{+}(u-k)(\varphi(u)-\varphi(k))\right] \\
& \leqslant \operatorname{sign}^{+}(u-k) g-\chi_{u=k} g^{-} \quad \text { в } \mathscr{D}^{\prime}\left(\Pi_{T}\right) .
\end{aligned}
$$

Применим метод “удвоения переменных", развитый С.Н. Кружковым в [10], [11]. Рассмотрим интегральное неравенство, соответствуюшее (50) для $u=u_{1}(t, x)$ с пробной функцией $f(t, x ; \tau, y) \geqslant 0$ в $\Pi_{T}(t, x) \times \Pi_{T}(\tau, y)$ и $k=\max \left(u_{2}(\tau, y), c\right)$, $c \in \mathbb{R} ;$ интегрируя это неравенство на $\Pi_{T}(\tau, y)$, получим

$$
\begin{gathered}
v_{t}+\operatorname{div}_{x} \varphi(v) \leqslant \operatorname{sign}^{+}\left(u_{1}-\max \left(u_{2}, c\right)\right) g(t, x, v) \\
-\chi_{u_{1}=\max \left(u_{2}, c\right)} g^{-}(t, x, v) \quad \text { в } \mathscr{D}^{\prime}\left(\Pi_{T} \times \Pi_{T}\right), \\
v=v(t, x ; \tau, y)=\max \left(u_{1}(t, x), u_{2}(\tau, y), c\right) .
\end{gathered}
$$

Меняя местами функции $u_{1}$ и $u_{2}$ и переменные $(t, x)$ и $(\tau, y)$, выводим аналогичное неравенство

$$
\begin{aligned}
v_{\tau}+\operatorname{div}_{y} \varphi(v) \leqslant & \operatorname{sign}^{+}\left(u_{2}-\max \left(u_{1}, c\right)\right) g(\tau, y, v) \\
& -\chi_{u_{2}=\max \left(u_{1}, c\right)} g^{-}(\tau, y, v) \text { в } \mathscr{D}^{\prime}\left(\Pi_{T} \times \Pi_{T}\right) .
\end{aligned}
$$

Пусть $w=w(t, x)=v(t, x ; t, x)=\max \left(u_{1}(t, x), u_{2}(t, x), c\right)$. Суммируя неравенства (51) и (52), после элементарных преобразований получим

$$
\begin{aligned}
\left(\frac{\partial}{\partial t}+\right. & \left.\frac{\partial}{\partial \tau}\right) v+\left(\operatorname{div}_{x}+\operatorname{div}_{y}\right) \varphi(v) \\
& \leqslant\left(\operatorname{sign}^{+}\left(u_{1}-\max \left(u_{2}, c\right)\right)+\operatorname{sign}^{+}\left(u_{2}-\max \left(u_{1}, c\right)\right)\right) g(t, x, w) \\
& \quad-\left(\chi_{u_{1}=\max \left(u_{2}, c\right)}+\chi_{u_{2}=\max \left(u_{1}, c\right)}\right) g^{-}(t, x, w)+R \quad \text { в } \mathscr{D}^{\prime}\left(\Pi_{T} \times \Pi_{T}\right)
\end{aligned}
$$

где

$$
\begin{aligned}
R= & R(t, x ; \tau, y)=\operatorname{sign}^{+}\left(u_{1}-\max \left(u_{2}, c\right)\right)(g(t, x, v)-g(t, x, w)) \\
& +\chi_{u_{1}=\max \left(u_{2}, c\right)}\left(g^{-}(t, x, w)-g^{-}(t, x, v)\right) \\
& +\operatorname{sign}^{+}\left(u_{2}-\max \left(u_{1}, c\right)\right)(g(\tau, y, v)-g(t, x, w)) \\
& +\chi_{u_{2}=\max \left(u_{1}, c\right)}\left(g^{-}(t, x, w)-g^{-}(\tau, y, v)\right) .
\end{aligned}
$$


Пусть $c \in D$, где $D \subset \mathbb{R}$ - множество значений $c$ таких, что множества уровня

$$
\left\{(t, x) \in \Pi_{T} \mid u_{1}(t, x)=c\right\}, \quad\left\{(\tau, y) \in \Pi_{T} \mid u_{2}(\tau, y)=c\right\}
$$

имеют нулевую меру Лебега. Ясно, что $D$ - множество полной меры и, в частности, $D$ всюду плотно на $\mathbb{R}$. Обозначим $p=p(t, x ; \tau, u)=\max \left(u_{1}(t, x), u_{2}(\tau, y)\right)$. При $c \in D$ п.в. на $\Pi_{T} \times \Pi_{T}$ имеем

$$
\begin{aligned}
\left(\operatorname{sign}^{+}(\right. & \left.\left.u_{1}-\max \left(u_{2}, c\right)\right)+\operatorname{sign}^{+}\left(u_{2}-\max \left(u_{1}, c\right)\right)\right) g \\
& -\left(\chi_{u_{1}=\max \left(u_{2}, c\right)}+\chi_{u_{2}=\max \left(u_{1}, c\right)}\right) g^{-} \\
= & \left(\chi_{\max \left(u_{1}, u_{2}\right)>c}-\chi_{u_{1}=u_{2}>c}\right) g-2 \chi_{u_{1}=u_{2}>c} g^{-} \\
\leqslant & \chi_{p>c} g-\chi_{u_{1}=u_{2}>c} g^{-} \leqslant \operatorname{sign}^{+}(p-c) g \\
\leqslant & Q_{h}=\left((p-c+h g)^{+}-(p-c)^{+}\right) / h \quad \forall h>0
\end{aligned}
$$

(здесь $g=g(t, x, w))$. Последнее неравенство следует из того, что $\forall \xi \in \mathbb{R}$ разностное отношение $\left((u+h \xi)^{+}-u^{+}\right) / h$ не убывает по $h>0$, ввиду выпуклости функции $u \mapsto u^{+}$и равенства

$$
\lim _{h \rightarrow 0+}\left((u+h \xi)^{+}-u^{+}\right) / h=\operatorname{sign}^{+}(u) \xi+\chi_{u=0} \xi^{+}
$$

(см. также доказательство теоремы 2). С учетом полученной оценки правой части неравенство (53) можно переписать в виде

$$
\left(\frac{\partial}{\partial t}+\frac{\partial}{\partial \tau}\right) v+\left(\operatorname{div}_{x}+\operatorname{div}_{y}\right) \varphi(v) \leqslant Q_{h}+R \text { в } \mathscr{D}^{\prime}\left(\Pi_{T} \times \Pi_{T}\right),
$$

где $h>0$ произвольно. Пусть $f(t, x) \in C_{0}^{\infty}\left(\Pi_{T}\right), f(t, x) \geqslant 0, \nu \in \mathbb{N}$. Положим

$$
\begin{aligned}
q(t, x ; \tau, y) & =f(t, x) k_{\nu}(t-\tau, x-y), \\
k_{\nu}(t-\tau, x-y) & =\delta_{\nu}(t-\tau) \prod_{i=1}^{n} \delta_{\nu}\left(x_{i}-y_{i}\right),
\end{aligned}
$$

где функции $\delta_{\nu}(s)$ определены при доказательстве предложения 1. Очевидно, при достаточно больших $\nu \in \mathbb{N}$ функция $q(t, x ; \tau, y)$ принадлежит $C_{0}^{\infty}\left(\Pi_{T} \times \Pi_{T}\right), q \geqslant 0$. Применяя неравенство (56) к пробной функции $q$, получим интегральное неравенство

$$
\int_{\Pi_{T}(t, x)} \int_{\Pi_{T}(\tau, y)}\left\{v f_{t}+\left(\varphi(v), \nabla_{x} f\right)+\left(Q_{h}+R\right) f\right\} k_{\nu}(t-\tau, x-y) d \tau d y d t d x \geqslant 0 .
$$

Пусть $g_{c}(t, x, u)=g(t, x, \max (u, c))$. Как следует из определения о.э.субр., при $i=1,2$

$$
g_{c}\left(t, x, u_{i}(t, x)\right)=\chi_{u_{i} \leqslant c} g(t, x, c)+\chi_{u_{i}>c} g\left(t, x, u_{i}(t, x)\right) \in L_{\mathrm{loc}}^{1}\left(\bar{\Pi}_{T}\right) .
$$


Далее, из неравенств

$$
\begin{aligned}
& \chi_{u_{1} \geqslant \max \left(u_{2}, c\right)}\left|g\left(t, x, u_{1}\right)-g(t, x, w)\right| \leqslant \\
& \leqslant \chi_{u_{2}(t, x)>u_{1}(t, x) \geqslant u_{2}(\tau, y)}\left|g_{c}\left(t, x, u_{1}(t, x)\right)-g_{c}\left(t, x, u_{2}(t, x)\right)\right| \\
& \begin{aligned}
& \chi_{u_{2} \geqslant \max \left(u_{1}, c\right)}\left|g\left(\tau, y, u_{2}\right)-g(t, x, w)\right| \leqslant \\
& \leqslant\left|g_{c}\left(\tau, y, u_{2}(\tau, y)\right)-g_{c}\left(t, x, u_{2}(t, x)\right)\right| \\
& \quad \quad \chi_{u_{2}(\tau, y) \geqslant u_{1}(t, x)>u_{2}(t, x)}\left|g_{c}\left(t, x, u_{2}(t, x)\right)-g_{c}\left(t, x, u_{1}(t, x)\right)\right|
\end{aligned}
\end{aligned}
$$

следует, что при $d=d(t, x)=u_{2}(t, x)-u_{1}(t, x), G=G(t, x)=\mid g_{c}\left(t, x, u_{1}(t, x)\right)-$ $g_{c}\left(t, x, u_{2}(t, x)\right) \mid$ справедлива оценка

$$
\begin{aligned}
& \int_{\Pi_{T}(\tau, y)}|R(t, x ; \tau, y)| k_{\nu}(t-\tau, x-y) d \tau d y \\
& \leqslant \chi_{d>0} G(t, x) \int_{\Pi_{T}(\tau, y)} \chi_{\left|u_{2}(t, x)-u_{2}(\tau, y)\right| \geqslant d} k_{\nu}(t-\tau, x-y) d \tau d y \\
&+\chi_{d<0} G(t, x) \int_{\Pi_{T}(\tau, y)} \chi_{\left|u_{2}(t, x)-u_{2}(\tau, y)\right| \geqslant-d} k_{\nu}(t-\tau, x-y) d \tau d y \\
&+\int_{\Pi_{T}(\tau, y)}\left|g_{c}\left(\tau, y, u_{2}(\tau, y)\right)-g_{c}\left(t, x, u_{2}(t, x)\right)\right| k_{\nu}(t-\tau, x-y) d \tau d y
\end{aligned}
$$

Кроме того, при $\varphi_{c}(u)=\varphi(\max (u, c))$

$$
\begin{aligned}
|\varphi(v)-\varphi(w)|=\mid \varphi_{c} & \left(\max \left(u_{1}(t, x), u_{2}(\tau, y)\right)\right)-\varphi_{c}\left(\max \left(u_{1}(t, x), u_{2}(t, x)\right)\right) \mid \\
\leqslant & \left|\varphi_{c}\left(u_{2}(\tau, y)\right)-\varphi_{c}\left(u_{2}(t, x)\right)\right| \\
& +\left|\varphi_{c}\left(u_{2}(\tau, y)\right)-\varphi_{c}\left(u_{1}(t, x)\right)\right| \chi_{u_{2}(\tau, y)>u_{1}(t, x)>u_{2}(t, x)} \\
& +\left|\varphi_{c}\left(u_{1}(t, x)\right)-\varphi_{c}\left(u_{2}(t, x)\right)\right| \chi_{u_{2}(t, x)>u_{1}(t, x)>u_{2}(\tau, y)} \leqslant \\
\leqslant & \left|\varphi_{c}\left(u_{2}(\tau, y)\right)-\varphi_{c}\left(u_{2}(t, x)\right)\right| \\
& +\left(\left|\varphi_{c}\left(u_{2}(\tau, y)\right)-\varphi_{c}\left(u_{2}(t, x)\right)\right|\right. \\
& \left.+\left|\varphi_{c}\left(u_{2}(t, x)\right)-\varphi_{c}\left(u_{1}(t, x)\right)\right|\right) \chi_{\left|u_{2}(t, x)-u_{2}(\tau, y)\right|>-d>0} \\
& +\left|\varphi_{c}\left(u_{1}(t, x)\right)-\varphi_{c}\left(u_{2}(t, x)\right)\right| \chi_{\left|u_{2}(t, x)-u_{2}(\tau, y)\right|>d>0} \\
\leqslant & 2\left|\varphi_{c}\left(u_{2}(\tau, y)\right)-\varphi_{c}\left(u_{2}(t, x)\right)\right| \\
& +\left|\varphi_{c}\left(u_{1}(t, x)\right)-\varphi_{c}\left(u_{2}(t, x)\right)\right| \chi_{\left|u_{2}(t, x)-u_{2}(\tau, y)\right|>|d|>0 .}
\end{aligned}
$$

Очевидны также оценки

$$
\begin{aligned}
|v(t, x ; \tau, y)-v(t, x ; t, x)| & \leqslant\left|u_{2}(\tau, y)-u_{2}(t, x)\right| \\
\left|Q_{h}(t, x ; \tau, y)-Q_{h}(t, x ; t, x)\right| & \leqslant \frac{2}{h}|p(t, x ; \tau, y)-p(t, x ; t, x)| \\
& \leqslant \frac{2}{h}\left|u_{2}(\tau, y)-u_{2}(t, x)\right| .
\end{aligned}
$$

Из (58)-(61) следует предельное соотношение

$$
\begin{gathered}
\int_{\Pi_{T}(\tau, y)}\left\{v f_{t}+\left(\varphi(v), \nabla_{x} f\right)+\left[Q_{h}+R\right] f\right\} k_{\nu}(t-\tau, x-y) d \tau d y \\
\left.\underset{\nu \rightarrow \infty}{\longrightarrow}\left\{v f_{t}+\left(\varphi(v), \nabla_{x} f\right)+Q_{h} f\right\}\right|_{\tau=t, y=x}
\end{gathered}
$$


на множестве полной меры значений $(t, x)$, являюшихся общими точками Лебега функций $u_{2}(t, x), g_{c}\left(t, x, u_{2}(t, x)\right), \varphi_{c}\left(t, x, u_{2}(t, x)\right)$. При этом (заметим, что $\left|Q_{h}\right| \leqslant$ $\left.\left|g_{c}(t, x, w)\right|\right)$

$$
\begin{aligned}
&\left|\int_{\Pi_{T}(\tau, y)}\left\{v f_{t}+\left(\varphi(v), \nabla_{x} f\right)+\left[Q_{h}+R\right] f\right\} k_{\nu}(t-\tau, x-y) d \tau d y\right| \\
& \leqslant\left(\left|u_{1}(t, x)\right|+\left(\left|u_{2}\right| * k_{\nu}\right)(t, x)\right)\left|f_{t}(t, x)\right| \\
&+\left(\left|\varphi\left(u_{1}(t, x)\right)\right|+\left(\left|\varphi\left(u_{2}\right)\right| * k_{\nu}\right)(t, x)\right)\left|\nabla_{x} f(t, x)\right| \\
&+|f(t, x)|\left[\left|g_{c}(t, x, w)\right|+|G(t, x)|+\left|g_{c}\right|+\left(\left|g_{c}\right| * k_{\nu}\right)(t, x)\right], \\
& g_{c}=g_{c}\left(t, x, u_{2}(t, x)\right),
\end{aligned}
$$

где *- операция свертки. Так как последовательность функций в правой части полученной оценки сходится при $\nu \rightarrow \infty$ в $L^{1}\left(\bar{\Pi}_{T}\right)$ и выполнено предельное соотношение (62), то по лемме 1

$$
\begin{aligned}
& \int_{\Pi_{T}(\tau, y)}\left\{v f_{t}+\right.\left.\left(\varphi(v), \nabla_{x} f\right)+\left[Q_{h}+R\right] f\right\} k_{\nu}(t-\tau, x-y) d \tau d y \\
& \underset{\nu \rightarrow \infty}{\longrightarrow}\left\{w f_{t}+\left(\varphi(w), \nabla_{x} f\right)+Q_{h} f\right\} \quad \text { в } L^{1}\left(\Pi_{T}\right)
\end{aligned}
$$

где $Q_{h}=Q_{h}(t, x ; t, x)=\left((u-c+h g)^{+}-(u-c)^{+}\right) / h, g=g(t, x, w(t, x))$, $u=p(t, x ; t, x)=\max \left(u_{1}(t, x), u_{2}(t, x)\right)$ и из соотношения $(57)$ в пределе при $\nu \rightarrow \infty$ следует неравенство

$$
\begin{gathered}
\int_{\Pi_{T}}\left\{w f_{t}+\left(\varphi(w), \nabla_{x} f\right)+Q_{h} f\right\} d t d x \geqslant 0, \\
w=\max \left(u(t, x), u_{2}(t, x), c\right)=\max (u(t, x), c), \\
Q_{h}=Q_{h}(t, x ; t, x)=\left((u-c+h g)^{+}-(u-c)^{+}\right) / h, \\
g=g(t, x, w(t, x)), \quad f=f(t, x) .
\end{gathered}
$$

Переходя здесь к пределу при $h \rightarrow 0+$, с учетом соотношения (55) и равенства $\chi_{u=c}(t, x)=0$ п.в. на $\Pi_{T}$ при $c \in D$, получим, что для любого $c \in D$

$$
\max (u, c)_{t}+\operatorname{div}_{x} \varphi(\max (u, c)) \leqslant \operatorname{sign}^{+}(u-c) g \text { в } \mathscr{D}^{\prime}\left(\Pi_{T}\right) .
$$

Заметим, что $w=u$ при $u>c$ и в последнем неравенстве $g=g(t, x, u)$. Ввиду равенств $(u-c)^{+}=\max (u, c)-c, \operatorname{sign}^{+}(u-c)(\varphi(u)-\varphi(c))=\varphi(\max (u, c))-\varphi(c)$, справедливо определяюшее соотношение $(20)$

$$
\begin{gathered}
\left((u-k)^{+}\right)_{t}+\operatorname{div}_{x}\left[\operatorname{sign}^{+}(u-k)(\varphi(u)-\varphi(k))\right] \\
\leqslant \operatorname{sign}^{+}(u-k) g \text { в } \mathscr{D}^{\prime}\left(\Pi_{T}\right)
\end{gathered}
$$

для всех $k=c \in D$. Тогда, с учетом предложения 2 , это соотношение верно для всех $k \in \mathbb{R}$.

Далее, из оценки $\left(u-u_{0}\right)^{+} \leqslant\left(u_{1}-u_{0}\right)^{+}+\left(u_{2}-u_{0}\right)^{+}\left(u_{0}=u_{0}(x)-\right.$ начальная функция) и начальных условий для о.э.субр. $u_{1}, u_{2}$ вытекает требуемое предельное соотношение

$$
\underset{t \rightarrow 0+}{\operatorname{ess}} \lim \left(u(t, x)-u_{0}(x)\right)^{+}=0 \quad \text { в } L_{\mathrm{loc}}^{1}\left(\mathbb{R}^{n}\right) .
$$

Итак, $u(t, x)=\max \left(u_{1}(t, x), u_{2}(t, x)\right)$ - о.э.субр. задачи (1), (2). Теорема доказана. 
Tеорема 5. Существует наибольшее о.э.субр. $v=J^{+}\left(u_{0}, g\right)$ и наименьшее о.э.суперр. $w=J^{-}\left(u_{0}, g\right)$ задачи (1), (2).

ДокАЗАТЕЛЬСтво. По теореме 3 существует о.э.р. задачи (1), (2), которое одновременно является о.э.субр. и о.э.суперр. этой задачи. Таким образом, множества о.э.субр. и о.э.суперр. задачи (1), (2) не пусты.

Для любой функции $u \in L_{\mathrm{loc}}^{1}\left(\bar{\Pi}_{T}\right)$ определим величину

$$
d(u)=\sum_{r=1}^{\infty} 2^{-r} \operatorname{arctg} p_{r}(u), \quad p_{r}(u)=\int_{\Pi_{T(1-1 / r)}} u(t, x) p(|x|-r) d t d x,
$$

где $p(s) \in C^{\infty}(\mathbb{R})$ - невозрастаюшая функция такая, что $p(s)=1$ при $s \leqslant 0$ и $p(s)=$ 0 при $s \geqslant 1$. Ясно, что $d(u)$ - возрастаюшая, ограниченная и непрерывная функция на $L_{\mathrm{loc}}^{1}\left(\bar{\Pi}_{T}\right)$. Пусть $C=\sup d(u)$, где $u=u(t, x)$ "пробегает" все о.э.субр. задачи $(1),(2)$. Тогда для любого натурального $k$ найдется такое о.э.субр. $u_{k}=u_{k}(t, x)$, что $d\left(u_{k}\right)>C-1 / k$. Пусть $v_{k}=\max \left(u_{1}, \ldots, u_{k}\right)$. Как следует из теоремы 4 , $v_{k}=v_{k}(t, x)$ - о.э.субр. задачи (1), (2). По построению $u_{k} \leqslant v_{k} \leqslant v_{k+1} \quad \forall k \in \mathbb{N}$ и $d\left(v_{k}\right) \geqslant d\left(u_{k}\right)>C-1 / k$. Из монотонности последовательности $v_{k}(t, x)$ следует сушествование предельной функции

$$
v(t, x)=\lim _{k \rightarrow \infty} v_{k}(t, x) \leqslant+\infty
$$

Далее, по предложению 3 при $u=v_{k}$ справедлива оценка (26), из которой, в свою очередь, легко выводится, что $\forall R>0$

$$
\begin{aligned}
\int_{\Pi_{T}}\left(v_{k}\right)^{+}(t, x) p(|x|-R) d t d x \leqslant & M(R)=T e^{L T} \int_{\mathbb{R}^{n}}\left(u_{0}^{+}(x)+2\right) p\left(|x|-C_{0} T-R\right) d x \\
& +T e^{L T} \int_{\Pi_{T}} g^{+}(t, x, 0) p\left(|x|-C_{0}(T-t)-R\right) d t d x
\end{aligned}
$$

откуда в пределе при $k \rightarrow \infty$ имеем

$$
\int_{\Pi_{T}} v^{+}(t, x) p(|x|-R) d t d x \leqslant M(R) .
$$

Учитывая также неравенство $v^{-}(t, x) \leqslant u_{1}^{-}(t, x)$, получаем, что $v(t, x) \in L_{\text {loc }}^{1}\left(\Pi_{T}\right)$ и $v_{k} \rightarrow v$ при $k \rightarrow \infty$ в $L_{\text {loc }}^{1}\left(\Pi_{T}\right)$. По теореме 1 тогда $v-$ о.э.субр. задачи $(1),(2)$. Покажем, что $v$ - наибольшее о.э.субр. этой задачи. Заметим, прежде всего, что $d(v) \geqslant d\left(v_{k}\right)$ для всех $k \in \mathbb{N}$, откуда следует, что $d(v)=C$. Пусть $u=u(t, x)-$ произвольное о.э.субр. задачи (1), (2). По теореме $4 w=\max (u, v)$ также является о.э.субр. этой задачи, причем $v \leqslant w, C=d(v) \leqslant d(w) \leqslant C$. Поэтому $\forall r \in \mathbb{N}$

$$
\begin{aligned}
p_{r}(v) & =\int_{\Pi_{T(1-1 / r)}} v(t, x) p(|x|-r) d t d x=p_{r}(w) \\
& =\int_{\Pi_{T(1-1 / r)}} w(t, x) p(|x|-r) d t d x, \quad v(t, x) \leqslant w(t, x),
\end{aligned}
$$

и, значит, $v(t, x)=w(t, x)$ п.в. на $\Pi_{T}$, что эквивалентно неравенству $v(t, x) \geqslant$ $u(t, x)$ п.в. на $\Pi_{T}$. Итак, $v(t, x)$ - наибольшее о.э.субр. задачи $(1),(2)$. 
По замечанию 2, b) если $v$ - наибольшее о.э.субр. задачи

$$
u_{t}-\operatorname{div}_{x} \varphi(-u)=-g(t, x,-u), \quad u(0, x)=-u_{0}(x)
$$

то $w=-v$ будет наименьшим о.э.суперр. исходной задачи. Теорема полностью доказана.

Следуя схеме доказательства теоремы 5 , легко установить более обший результат: точная верхняя грань любого мнохества о.э.субр. и точная нижсняя грань любого множсества о.э.суперр. задачи (1), (2) являются соответственно о.э.субр. и о.э.суперр. этой задачи.

Заметим, что теоремы 4, 5 для класса ограниченных о.э.субр. и о.э.суперр. доказаны в работе [19].

ТЕОРЕма 6. Наибольшее о.э.субр. и наименьшее о.э.суперр. задачи (1), (2) являются о.э.р. этой задачи. В частности, $v=J^{+}\left(u_{0}, g\right) u w=J^{-}\left(u_{0}, g\right)$ являются соответственно наибольиим и наименьиим о.э.р. задачи (1), (2).

ДокАЗАТЕльСтво. Ввиду замечания 2, b) достаточно доказать утверждение, касающееся наибольшего о.э.субр. $v=v(t, x)$. При $k \in \mathbb{N}$ рассмотрим задачу Коши для уравнения

$$
u_{t}+\operatorname{div}_{x} \varphi(u)=g_{k}=g(t, x, u)+k(v(t, x)-u)^{+}
$$

с начальным условием (2). По теореме 3 существует о.э.р. задачи $(63),(2) u_{k}=$ $u_{k}(t, x)=\mathscr{F}\left(u_{0}, g_{k}\right)$, причем по свойству монотонности $u_{k+1} \geqslant u_{k}$ п.в. на $\Pi_{T}$ для любого $k \in \mathbb{N}$. Так как $g_{k}(t, x, v)=g(t, x, v)$ при $u \geqslant v$, то $v$ является также наибольшим о.э.субр. задачи $(63),(2)$ и, в частности, $v \geqslant u_{k}$ п.в. на $\Pi_{T}, k \in \mathbb{N}$. Из монотонности последовательности $u_{k}, k \in \mathbb{N}$, и оценки $u_{k} \leqslant v$ следует, что $u_{k} \rightarrow u=u(t, x)$ при $k \rightarrow \infty$ п.в. на $\Pi_{T}$ и в $L_{\text {loc }}^{1}\left(\Pi_{T}\right)$, причем $u \leqslant v$ п.в. на П $T$. По следствию 1 для любой пробной функции $f=f(t, x) \in C_{0}^{\infty}\left(\bar{\Pi}_{T}\right), f \geqslant 0$, имеем

$$
\begin{aligned}
I_{k} & =\int_{\Pi_{T}}\left[u_{k} f_{t}+\left(\varphi\left(u_{k}\right), \nabla_{x} f\right)+g\left(t, x, u_{k}\right) f\right] d t d x+\int_{\mathbb{R}^{n}} u_{0}(x) f(0, x) d x \\
& =-k \int_{\Pi_{T}}\left(v-u_{k}\right)^{+} f d t d x
\end{aligned}
$$

Поскольку $\forall k \in \mathbb{N} u_{1} \leqslant u_{k} \leqslant v$ п.в. на $\Pi_{T}$, то $\left|u_{k}\right| \leqslant\left|u_{1}\right|+|v| \in L_{\text {lос }}^{1}\left(\bar{\Pi}_{T}\right)$ и последовательность $I_{k}$ ограничена: $\left|I_{k}\right| \leqslant C=C_{f}=$ const. Тогда из (64) следует, что

$$
\int_{\Pi_{T}}\left(v-u_{k}\right)^{+} f d t d x \leqslant C / k \underset{k \rightarrow \infty}{\longrightarrow} 0
$$

для любой функции $f \in C_{0}^{\infty}\left(\bar{\Pi}_{T}\right), f \geqslant 0$, т.е. $u_{k} \rightarrow v$ в $L_{\mathrm{loc}}^{1}\left(\bar{\Pi}_{T}\right)$ и, значит, $u=v$ п.в. на $\Pi_{T}$. Заметим также, что из неравенства $g(t, x, v)-g\left(t, x, u_{k}\right) \leqslant L\left(v-u_{k}\right)$ следует, что $g^{+}(t, x, v) \in L_{\text {loc }}^{1}\left(\bar{\Pi}_{T}\right)$ и

$$
\int_{\Pi_{T}} g(t, x, v) f d t d x \leqslant \liminf _{k \rightarrow \infty} \int_{\Pi_{T}} g\left(t, x, u_{k}\right) f d t d x
$$


Переходя в равенстве (64) к пределу при $k \rightarrow \infty$, получим соотношение

$$
\begin{aligned}
\lim _{k \rightarrow \infty} k \int_{\Pi_{T}}\left(v-u_{k}\right)^{+} f d t d x \leqslant & -\int_{\Pi_{T}}\left[v f_{t}+\left(\varphi(v), \nabla_{x} f\right)+g(t, x, v) f\right] d t d x \\
& -\int_{\mathbb{R}^{n}} u_{0}(x) f(0, x) d x \leqslant 0,
\end{aligned}
$$

так как $v$ - о.э.субр. задачи (1), (2) (см. замечание 3, неравенство (23)). Ввиду произвольности $f \in C_{0}^{\infty}\left(\bar{\Pi}_{T}\right), f \geqslant 0, k\left(v-u_{k}\right)^{+} \rightarrow 0$ в $L_{\mathrm{loc}}^{1}\left(\bar{\Pi}_{T}\right)$. Тогда при $k \rightarrow \infty u_{k} \rightarrow v$ и $\bar{g}_{k}=g(t, x, u)+k\left(v(t, x)-u_{k}(t, x)\right)^{+} \rightarrow g(t, x, u)$ в $L_{\text {lос }}^{1}\left(\bar{\Pi}_{T}\right)$ при любом фиксированном $u \in \mathbb{R}$. Поскольку $u_{k}-$ о.э.р. задачи $(1),(2)$ с входными данными $\left(u_{0}, \bar{g}_{k}\right)$, то по теореме 1 функция $v(t, x)-$ о.э.р. исходной задачи. Теорема доказана.

Отметим, что для класса ограниченных о.э.р., когда $u_{0} \in L^{\infty}\left(\mathbb{R}^{n}\right), g=g(t, x) \in$ $L^{1}\left((0, T), L^{\infty}\left(\mathbb{R}^{n}\right)\right)$, результат о сушествовании наибольшего и наименьшего о.э.р. установлен также в [20].

СЛЕДСТВИЕ 4. Пусть $u_{0}, v_{0} \in L_{\text {loc }}^{1}\left(\mathbb{R}^{n}\right), g, h \in S, u_{0} \leqslant v_{0}$ n.в. нa $\mathbb{R}^{n}, g \leqslant h$ n.в. на $\Pi_{T} \times \mathbb{R} u u^{ \pm}=J^{ \pm}\left(u_{0}, g\right), v^{ \pm}=J^{ \pm}\left(v_{0}, h\right)$. Тогда $u^{+} \leqslant v^{+}, u^{-} \leqslant v^{-}$ n.в. на $\Pi_{T}$.

ДоказАТЕЛЬСтво. Ясно, что $u^{+}$- о.э.субр. задачи $(1),(2)$ с входными данными $\left(v_{0}, h\right)$, и так как $v^{+}-$наибольшее о.э.субр. этой задачи, то $u^{+} \leqslant v^{+}$п.в. на $\Pi_{T}$. Далее, поскольку $v^{-}-$о.э.суперр. задачи $(1),(2)$ с входными данными $\left(u_{0}, g\right)$, а $u^{-}$- наименьшее о.э.суперр. этой задачи, то $u^{-} \leqslant v^{-}$п.в. на $\Pi_{T}$.

\section{§ 4. Принцип сравнения и единственность о.э.р.}

Как было отмечено выше, в обшем случае о.э.р. задачи (1), (2) неединственно. Первые достаточные условия единственности о.э.р. в классе ограниченных суммируемых функций найдены в [12], [13] и распространены на класс произвольных ограниченных о.э.р. в [14], [15]. Эти условия формулируются в терминах модулей непрерывностей функций потока $\varphi_{i}(u), i=1, \ldots, n$, и аналогичны известному условию Осгуда в теории обыкновенных дифференциальных уравнений.

После соответствуюших изменений условия из [14], [15] подходят для доказательства единственности о.э.р. (принщипа сравнения для о.э.субр. и о.э.суперр.) задачи $(1),(2)$ и в рассматриваемом случае локально суммируемых о.э.р.

Прежде всего, необходимо видоизменить основную лемму 1.1,b) из работы [6] и, в частности, избавиться от требуемого в [6] экспоненциального ограничения на рост функции $w(t, x)$. Итак, справедлива следующая

ЛЕмма 5. Пусть $\lambda_{i}(\varepsilon), i=1, \ldots, n,-$ набор положсительных функиий на $\mathbb{R}_{+}=(0,+\infty)$ таких, что существуют (возмохнно, бесконечные) предель

$$
\begin{gathered}
0<\lambda_{i}(0)=\lim _{\varepsilon \rightarrow 0+} \lambda_{i}(\varepsilon) \leqslant+\infty, \\
C=\liminf _{\varepsilon \rightarrow 0+} \varepsilon \prod_{i=1}^{n} \lambda_{i}(\varepsilon)<+\infty,
\end{gathered}
$$


причем $C=0$, если существует лишь одно значение $\lambda_{i}(0)=+\infty$. Предположим, ито $w, q \in L_{\mathrm{loc}}^{1}\left(\Pi_{T}\right), \quad q \in L^{1}\left(\Pi_{T}\right), \quad w_{0}(x) \in L^{1}\left(\mathbb{R}^{n}\right), \quad w, q \geqslant 0$ и для некоторой константы $L \geqslant 0$ справедливо неравенство

$$
\int_{\Pi_{T}}\left\{w f_{t}+\sum_{i=1}^{n}(w+\varepsilon) \lambda_{i}(\varepsilon)\left|f_{x_{i}}\right|+(L w+q) f\right\} d t d x \geqslant 0
$$

для всех $\varepsilon>0, f=f(t, x) \in C_{0}^{\infty}\left(\Pi_{T}\right), f \geqslant 0, u$

$$
\underset{t \rightarrow 0+}{\operatorname{ess}} \lim \left(w(t, x)-w_{0}(x)\right)^{+}=0 \quad \text { в } \quad L_{\mathrm{loc}}^{1}\left(\mathbb{R}^{n}\right) .
$$

Тогда $w(\tau, \cdot) \in L^{1}\left(\mathbb{R}^{n}\right)$ для n.в. $\tau \in(0, T)$ u

$$
\int_{\mathbb{R}^{n}} w(\tau, x) d x \leqslant e^{L \tau}\left(\int_{\mathbb{R}^{n}} w_{0}(x) d x+\int_{\Pi_{\tau}} q(t, x) d t d x\right) .
$$

ДОКАЗАТЕЛЬСТВО. Пусть $l$ - число индексов $i=1, \ldots, n$, для которых $\lambda_{i}(0)=$ $+\infty$. Если $l=0$, то можно заменить $\lambda_{i}(\varepsilon)$ на $\lambda_{i}(\varepsilon)+\varepsilon^{-\frac{1}{n+1}}$ так, чтобы условие $(66)$ выполнялось. Таким образом, можно предполагать, что $l \geqslant 1$. При необходимости изменив порядок координат, будем также предполагать, что $\lambda_{i}(0)=+\infty$ для $i=$ $1, \ldots, l$.

Пусть $p(s) \in C^{\infty}(\mathbb{R})$ - невозрастающая функция такая, что $p(s)=1$ при $s \leqslant 0$ и $p(s)=0$ при $s \geqslant 1$. Положим при $\bar{T}, R>0$

$$
\begin{gathered}
\xi(t, x)=\prod_{i=1}^{l} p\left(\left|x_{i}\right|-\lambda_{i}(\varepsilon)(\bar{T}-t)-R\right) \prod_{i=l+1}^{n} p\left(\left|x_{i}\right|-\lambda_{i}(\varepsilon)(T-t)-R\right), \\
\gamma_{\nu}(t)=\theta_{\nu}\left(t-t_{0}\right)-\theta_{\nu}\left(t-t_{1}\right), \quad f=f(t, x)=\xi(t, x) \gamma_{\nu}(t)
\end{gathered}
$$

здесь $0<t_{0}<t_{1}<\bar{T}, 1 / \nu<\bar{T}-t_{1}$, функции $\theta_{\nu}(t)=\int_{-\infty}^{t} \delta_{\nu}(s) d s$ определены при доказательстве предложения 1 .

Ясно, что $f(t, x) \in C_{0}^{\infty}\left(\Pi_{T}\right), f \geqslant 0$. Подставляя пробную функцию $f$ в неравенство (67), после элементарных преобразований получим

$$
\begin{aligned}
& \int_{0}^{T}\left(\int_{\mathbb{R}^{n}} w(t, x) \xi(t, x) d x\right)\left(\delta_{\nu}\left(t-t_{0}\right)-\delta_{\nu}\left(t-t_{1}\right)\right) d t \\
& \quad+\int_{\Pi_{T}}\left[w \xi_{t}+\sum_{i=1}^{n}(w+\varepsilon) \lambda_{i}(\varepsilon)\left|\xi_{x_{i}}\right|+(L w+q) \xi\right] \gamma_{\nu}(t) d t d x \geqslant 0 .
\end{aligned}
$$

Поскольку $\xi_{t}=-\sum_{i=1}^{n} \lambda_{i}(\varepsilon)\left|\xi_{x_{i}}\right|$, из (70) следует неравенство

$$
\begin{gathered}
\int_{0}^{T}\left(\int_{\mathbb{R}^{n}} w(t, x) \xi(t, x) d x\right) \delta_{\nu}\left(t-t_{1}\right) d t \leqslant \int_{0}^{T}\left(\int_{\mathbb{R}^{n}} w(t, x) \xi(t, x) d x\right) \delta_{\nu}\left(t-t_{0}\right) d t \\
\quad+\int_{\Pi_{T}}(L w+q) \xi \gamma_{\nu}(t) d t d x+\varepsilon \int_{\Pi_{T}}\left|\xi_{t}\right| \gamma_{\nu}(t) d t d x
\end{gathered}
$$


Легко видеть, что (с учетом неравенства $\xi(t, x) \leqslant \xi\left(t_{0}, x\right)$ при $\left.t>t_{0}\right)$

$$
\begin{aligned}
\int_{\Pi_{T}}\left|\xi_{t}\right| \gamma_{\nu}(t) d t d x & =-\int_{\Pi_{T}} \xi_{t} \gamma_{\nu}(t) d t d x=\int_{\Pi_{T}} \xi(t, x) \gamma_{\nu}^{\prime}(t) d t d x \\
& \leqslant \int_{\Pi_{T}} \xi(t, x) \delta_{\nu}\left(t-t_{0}\right) d t d x \leqslant \int_{\mathbb{R}^{n}} \xi\left(t_{0}, x\right) d x \\
& \leqslant \int_{\mathbb{R}^{n}} \prod_{i=1}^{l} \chi_{\left|x_{i}\right| \leqslant \lambda_{i}(\varepsilon)\left(\bar{T}-t_{0}\right)+R+1} \prod_{i=l+1}^{n} \chi_{\left|x_{i}\right| \leqslant \lambda_{i}(\varepsilon)\left(T-t_{0}\right)+R+1} d x
\end{aligned}
$$

и, значит,

$$
\varepsilon \int_{\Pi_{T}}\left|\xi_{t}\right| \gamma_{\nu}(t) d t d x \leqslant \alpha\left(t_{0}, \varepsilon\right) C(\varepsilon)
$$

где

$$
\begin{gathered}
C(\varepsilon)=\varepsilon \prod_{i=1}^{n} \lambda_{i}(\varepsilon), \\
\alpha\left(t_{0}, \varepsilon\right)=2^{n} \prod_{i=1}^{l}\left(\bar{T}-t_{0}+(R+1) / \lambda_{i}(\varepsilon)\right) \prod_{i=l+1}^{n}\left(T-t_{0}+(R+1) / \lambda_{i}(\varepsilon)\right) .
\end{gathered}
$$

Пусть $0<\lambda<\min \lambda_{i}(0)$. Тогда найдется последовательность $\varepsilon_{m}, m \in \mathbb{N}$, такая, что $\lambda_{i}\left(\varepsilon_{m}\right)>\lambda>0, \lim _{m \rightarrow \infty} \varepsilon_{m}=0$ и в соответствии с (66) $C\left(\varepsilon_{m}\right) \rightarrow C$ при $m \rightarrow \infty$. Пусть, далее, $\mathscr{E}_{1}-$ множество общих точек Лебега счетного семейства функций $F(t)=\int_{\mathbb{R}^{n}} w(t, x) \xi(t, x) d x$, где параметры $R, \bar{T}$ рациональны, а $\varepsilon=\varepsilon_{m}$, $m \in \mathbb{N}$. Тогда $\mathscr{E}_{1}-$ множество полной меры Лебега на $(0, T)$, и если $t_{0}, t_{1} \in \mathscr{E}_{1}$, то из $(71)$ в пределе при $\nu \rightarrow \infty$ (с учетом оценки последнего интеграла) получим

$$
\begin{aligned}
\int_{\mathbb{R}^{n}} w\left(t_{1}, x\right) \xi\left(t_{1}, x\right) d x \leqslant & \int_{\mathbb{R}^{n}} w\left(t_{0}, x\right) \xi\left(t_{0}, x\right) d x \\
& +\int_{\left[t_{0}, t_{1}\right] \times \mathbb{R}^{n}}(L w+q) \xi d t d x+\alpha\left(t_{0}, \varepsilon\right) C(\varepsilon) .
\end{aligned}
$$

Пусть $\mathscr{E} \subset(0, T)$ - множество полной меры такое, что в соответствии с $(68)$ $w(t, \cdot) \in L_{\text {lос }}^{1}\left(\mathbb{R}^{n}\right)$ при $t \in \mathscr{E}$ и $\lim _{t \rightarrow 0+, t \in \mathscr{E}}\left(w(t, x)-w_{0}(x)\right)^{+}=0$ в $L_{\text {lос }}^{1}\left(\mathbb{R}^{n}\right)$, $t_{0} \in \mathscr{E}_{1} \cap \mathscr{E}$. Устремляя в $(72) t_{0}$ к нулю и учитывая, что

$$
\alpha\left(t_{0}, \varepsilon\right) \underset{t_{0} \rightarrow 0}{\longrightarrow} \alpha(0, \varepsilon) \leqslant \alpha(\varepsilon)=2^{n}(T+(R+1) / \lambda)^{n-l} \prod_{i=1}^{l}\left(\bar{T}+(R+1) / \lambda_{i}(\varepsilon)\right)
$$

получим, что для всех $\bar{T}, R \in \mathbb{Q}, \varepsilon=\varepsilon_{m}, m \in \mathbb{N}, \tau \in \mathscr{E}_{1}, \tau<\bar{T}$ (для удобства мы заменили $t_{1}$ на $\tau$ ) вьполнено

$$
\begin{aligned}
\int_{\mathbb{R}^{n}} w(\tau, x) \xi(\tau, x) d x \leqslant & \int_{\mathbb{R}^{n}} w_{0}(x) \xi(0, x) d x+\int_{\Pi_{\tau}}(L w+q) \xi(t, x) d t d x+\alpha(\varepsilon) C(\varepsilon) \\
\leqslant & \int_{\mathbb{R}^{n}} w_{0}(x) d x+\int_{\Pi_{\tau}} q(t, x) d t d x+\alpha(\varepsilon) C(\varepsilon) \\
& +L \int_{0}^{\tau} \int_{\mathbb{R}^{n}} w(t, x) \xi(t, x) d x d t
\end{aligned}
$$


По лемме Гронуолла получаем, что

$$
\int_{\mathbb{R}^{n}} w(\tau, x) \xi(\tau, x) d x \leqslant e^{L \tau}\left(\int_{\mathbb{R}^{n}} w_{0}(x) d x+\int_{\Pi_{\tau}} q(t, x) d t d x+\alpha(\varepsilon) C(\varepsilon)\right) .
$$

Перейдем в этом неравенстве к пределу при $\varepsilon=\varepsilon_{m} \rightarrow 0$. Так как $\lambda_{i}(\varepsilon) \rightarrow$ $\lambda_{i}(0)=+\infty, i=1, \ldots, l$, то при $\bar{T}>t$ вьполнены предельные соотношения $p\left(\left|x_{i}\right|-\lambda_{i}(\varepsilon)(\bar{T}-t)-R\right) \rightarrow 1, \alpha(\varepsilon) \rightarrow \eta \bar{T}^{l}$, где $\eta=2^{n}(T+(R+1) / \lambda)^{n-l}$. Используя лемму Фату, получим из (74) в пределе оценку

$$
\int_{\mathbb{R}^{n}} w(\tau, x) \bar{\xi}(\tau, x) d x \leqslant e^{L \tau}\left(\int_{\mathbb{R}^{n}} w_{0}(x) d x+\int_{\Pi_{\tau}} q d t d x+\eta C \bar{T}^{l}\right),
$$

где $\bar{\xi}(\tau, x)=\prod_{i=l+1}^{n} p\left(\left|x_{i}\right|-\lambda_{i}(0)(T-\tau)-R\right), \bar{T}>\tau$. Из этой оценки в пределе при $\bar{T} \rightarrow \tau$ следует, что

$$
\int_{\mathbb{R}^{n}} w(\tau, x) \bar{\xi}(\tau, x) d x \leqslant e^{L \tau}\left(\int_{\mathbb{R}^{n}} w_{0}(x) d x+\int_{\Pi_{\tau}} q d t d x+\eta C \tau^{l}\right),
$$

в частности, $\forall \tau \in \mathscr{E} 1$

$$
\int_{\mathbb{R}^{n}} w(\tau, x) \bar{\xi}(\tau, x) d x \leqslant \text { const }=e^{L T}\left(\int_{\mathbb{R}^{n}} w_{0}(x) d x+\int_{\Pi_{T}} q d t d x+\eta C T^{l}\right)<\infty .
$$

Если $l=1$, то по условию $C=0$ и в пределе при $R \rightarrow+\infty$ из неравенства $(75)$ вытекает, что $\forall \tau \in \mathscr{E}_{1}$

$$
\int_{\mathbb{R}^{n}} w(\tau, x) d x \leqslant e^{L \tau}\left(\int_{\mathbb{R}^{n}} w_{0}(x) d x+\int_{\Pi_{\tau}} q d t d x\right),
$$

что и нужно было доказать. Если же $l>1$, то перейдем в $(72)$ к пределу сначала при $\varepsilon_{m} \rightarrow 0$, а затем при $\bar{T} \rightarrow t_{1}$. Учитывая, что

$$
\lim _{m \rightarrow \infty} \alpha\left(t_{0}, \varepsilon_{m}\right) \leqslant \eta\left(\bar{T}-t_{0}\right)^{l}
$$

придем к неравенству

$$
\begin{aligned}
\int_{\mathbb{R}^{n}} w\left(t_{1}, x\right) \bar{\xi}\left(t_{1}, x\right) d x \leqslant & \int_{\mathbb{R}^{n}} w\left(t_{0}, x\right) \bar{\xi}\left(t_{0}, x\right) d x+\int_{\left[t_{0}, t_{1}\right] \times \mathbb{R}^{n}}(L w+q) \bar{\xi} d t d x \\
& +\eta\left(t_{1}-t_{0}\right)^{l} \quad \forall t_{0}, t_{1} \in \mathscr{E}_{1}, \quad t_{1}>t_{0}
\end{aligned}
$$

При $H(\tau)=\int_{\mathbb{R}^{n}} w(\tau, x) \bar{\xi}(\tau, x) d x-\int_{\Pi_{\tau}}(L w+q) \bar{\xi} d t d x$ неравенство (76) можно переписать в виде $H\left(t_{1}\right)-H\left(t_{0}\right) \leqslant \eta\left(t_{1}-t_{0}\right)^{l}$, и так как $l>1$, то отсюда вытекает, что $H\left(t_{1}\right) \leqslant H\left(t_{0}\right) \forall t_{0}, t_{1} \in \mathscr{E}_{1}, t_{1}>t_{0}$. Перейдем в этом неравенстве к пределу при $t_{0} \rightarrow 0$. Как следует из (75),

$$
\limsup _{t_{0} \rightarrow 0, t_{0} \in \mathscr{E}_{1}} H\left(t_{0}\right) \leqslant \int_{\mathbb{R}^{n}} w_{0}(x) d x
$$


и мы получаем, что $H(\tau) \leqslant \int_{\mathbb{R}^{n}} w_{0}(x) d x \forall \tau \in \mathscr{E}_{1}$, т.е.

$$
\begin{aligned}
\int_{\mathbb{R}^{n}} w(\tau, x) \bar{\xi}(\tau, x) d x \leqslant & \int_{\mathbb{R}^{n}} w_{0}(x) d x+\int_{\Pi_{t}}(L w+q) \bar{\xi} d t d x \\
\leqslant & \int_{\mathbb{R}^{n}} w_{0}(x) d x+\int_{\Pi_{\tau}} q(t, x) d t d x \\
& +L \int_{0}^{\tau} \int_{\mathbb{R}^{n}} w(t, x) \bar{\xi}(t, x) d x d t
\end{aligned}
$$

По лемме Гронуолла $\forall \tau \in \mathscr{E}_{1}$

$$
\int_{\mathbb{R}^{n}} w(\tau, x) \bar{\xi}(\tau, x) d x \leqslant e^{L \tau}\left(\int_{\mathbb{R}^{n}} w_{0}(x) d x+\int_{\Pi_{\tau}} q(t, x) d t d x\right),
$$

и для завершения доказательства остается перейти к пределу при $R \rightarrow \infty$ в последнем неравенстве, с использованием леммы Фату. Лемма доказана.

Следует отметить, что лемма 5, по существу, доказана в [16].

Для доказательства теоремы единственности ключевую роль играет следующий результат из [5], [6] (см. также [11], [4], [7], [2]).

ЛЕмма 6. Пусть $u$ - о.э.субр., v - о.ә.суперр. задачи (1), (2) с входнымми данными $\left(u_{0}, g\right),\left(v_{0}, h\right)$ соответственно, $g=g(t, x), \quad h=h(t, x), \quad g, h \in$ $L_{\mathrm{loc}}^{1}\left(\Pi_{T}\right)$. Тогда

$$
\begin{aligned}
\frac{\partial}{\partial t}(u-v)^{+} & +\operatorname{div}_{x}\left[\operatorname{sign}^{+}(u-v)(\varphi(u)-\varphi(v))\right] \\
& \leqslant(g-h) \chi_{u>v}+(g-h)^{+} \chi_{u=v} \leqslant(g-h)^{+} \chi_{u \geqslant v} \quad \text { в } \quad \mathscr{D}^{\prime}\left(\Pi_{T}\right) .
\end{aligned}
$$

ТЕОРема 7. Предположим, что существуют $\omega_{i}(r)$ - неубиваюшие субаддитивные функции на $\mathbb{R}_{+}=(0,+\infty), \omega_{i}(r)>0$ при $r>0$, такие, что

$$
\begin{gathered}
\left|\varphi_{i}(u)-\varphi_{i}(v)\right| \leqslant \omega_{i}(|u-v|) \quad \forall u, v \in \mathbb{R}, \quad i=1, \ldots, n, \\
\liminf _{r \rightarrow 0+} r^{1-n} \prod_{i=1}^{n} \omega_{i}(r)<\infty .
\end{gathered}
$$

Пусть $u=u(t, x)$ - о.ә.субр., $v=v(t, x)$ - о.ә.суперр. задачи (1), (2) с входньми данньлми $\left(u_{0}, g\right),\left(v_{0}, h\right)$ соответственно такие, что $g(t, x, u), h(t, x, v) \in$ $L_{\text {loc }}^{1}\left(\Pi_{T}\right) u\left(u_{0}-v_{0}\right)^{+} \in L^{1}\left(\mathbb{R}^{n}\right)$,

$$
\begin{gathered}
q=q(t, x, u, v)=\chi_{u \geqslant v} \min \left\{(g(t, x, v)-h(t, x, v))^{+},\right. \\
\left.(g(t, x, u)-h(t, x, u))^{+}\right\} \in L^{1}\left(\Pi_{T}\right) .
\end{gathered}
$$

Тогда для п.в. $\tau \in(0, T)$

$$
\begin{aligned}
\int_{\mathbb{R}^{n}}(u(\tau, x)-v(\tau, x))^{+} d x \leqslant e^{L \tau} & \left(\int_{\mathbb{R}^{n}}\left(u_{0}(x)-v_{0}(x)\right)^{+} d x\right. \\
& \left.+\int_{\Pi_{\tau}} q(t, x, u, v) d t d x\right)
\end{aligned}
$$

əде $L=\max (L(g), L(h))$. 
ДокАЗАТЕЛЬСтво. Заметим, что ввиду (4)

$$
\begin{aligned}
(g-h)^{+} \chi_{u} \geqslant v & \leqslant(g(t, x, u)-g(t, x, v))^{+} \chi_{u \geqslant v}+(g(t, x, v)-h(t, x, v))^{+} \chi_{u} \geqslant v \\
& \leqslant L(u-v)^{+}+(g(t, x, v)-h(t, x, v))^{+} \chi_{u \geqslant v}, \\
(g-h)^{+} \chi_{u} \geqslant v & \leqslant(h(t, x, u)-h(t, x, v))^{+} \chi_{u \geqslant v}+(g(t, x, u)-h(t, x, u))^{+} \chi_{u} \geqslant v \\
& \leqslant L(u-v)^{+}+(g(t, x, u)-h(t, x, u))^{+} \chi_{u} \geqslant v,
\end{aligned}
$$

откуда следует, что

$$
(g-h)^{+} \chi_{u \geqslant v} \leqslant L(u-v)^{+}+q(t, x, u, v) \in L_{\mathrm{loc}}^{1}\left(\Pi_{T}\right) .
$$

Ясно, что $u$ - о.э.субр., а $v$ - о.э.суперр. задачи (1), (2) с входными данными $\left(u_{0}, g\right),\left(v_{0}, h\right)$ соответственно, где $g=g(t, x, u(t, x)), h=h(t, x, v(t, x))$. Тогда по лемме 6

$$
\begin{aligned}
& \frac{\partial}{\partial t}(u-v)^{+}+\operatorname{div}_{x}\left[\operatorname{sign}^{+}(u-v)(\varphi(u)-\varphi(v))\right] \\
& \quad \leqslant(g-h)^{+} \chi_{u} \geqslant v \leqslant L(u-v)^{+}+q \text { в } \quad \mathscr{D}^{\prime}\left(\Pi_{T}\right) .
\end{aligned}
$$

Применяя это неравенство к пробной функции $f=f(t, x) \in C_{0}^{\infty}\left(\Pi_{T}\right), f \geqslant 0$, получим

$$
\int_{\Pi_{T}}(u-v)^{+} f_{t}+\operatorname{sign}^{+}(u-v)\left(\varphi(u)-\varphi(v), \nabla_{x} f\right)+\left(L(u-v)^{+}+q\right) f d t d x \geqslant 0 .
$$

Из (78) следует оценка

$$
\left|\operatorname{sign}^{+}(u-v)\left(\varphi(u)-\varphi(v), \nabla_{x} f\right)\right| \leqslant \sum_{i=1}^{n} \omega_{i}\left((u-v)^{+}\right)\left|f_{x_{i}}\right| .
$$

Пусть $\varepsilon>0$. Представим произвольное $r \geqslant 0$ в виде $r=k \varepsilon+s$, где $k \in \mathbb{N} \cup\{0\}$ и $0 \leqslant s<\varepsilon$. Так как функции $\omega_{i}(r)$ не убывают и субаддитивны, то

$$
\omega_{i}(r) \leqslant \omega_{i}((k+1) \varepsilon) \leqslant(k+1) \omega_{i}(\varepsilon) \leqslant(r+\varepsilon) \omega_{i}(\varepsilon) / \varepsilon=\lambda_{i}(\varepsilon)(r+\varepsilon)
$$

при $\lambda_{i}(\varepsilon)=\omega_{i}(\varepsilon) / \varepsilon$. Тогда из (82) с учетом (83) и (84) следует, что функция $w=(u-v)^{+}$удовлетворяет интегральному неравенству вида $(67)$

$$
\int_{\Pi_{T}}\left\{w f_{t}+\sum_{i=1}^{n}(w+\varepsilon) \lambda_{i}(\varepsilon)\left|f_{x_{i}}\right|+(L w+q) f\right\} d t d x \geqslant 0
$$

для всех $\varepsilon>0, f=f(t, x) \in C_{0}^{\infty}\left(\Pi_{T}\right), f \geqslant 0$, причем ввиду (79) функции $\lambda_{i}(\varepsilon)$ удовлетворяют предположениям леммы 5 . Заметим, что из (84) следует, что $\lambda_{i}(\varepsilon) \geqslant \omega_{i}(r) /(r+\varepsilon) \forall r>0$ и, следовательно, $\liminf _{\varepsilon \rightarrow 0+} \lambda_{i}(\varepsilon) \geqslant \lambda_{i}(r)=$ $\omega_{i}(r) / r>0$. Отсюда легко вытекает существование пределов $\lim _{\varepsilon \rightarrow 0+} \lambda_{i}(\varepsilon)>0$. Далее, если $w_{0}(x)=\left(u_{0}-v_{0}\right)^{+}$, то ввиду оценки $\left(w(t, x)-w_{0}(x)\right)^{+} \leqslant(u(t, x)-$ $\left.u_{0}(x)\right)^{+}+\left(v_{0}(x)-v(t, x)\right)^{+}$и начальных условий из определений 2 и 3 имеем

$$
\underset{t \rightarrow 0+}{\operatorname{ess}} \lim \left(w(t, x)-w_{0}(x)\right)^{+}=0 \quad \text { в } \quad L_{\mathrm{loc}}^{1}\left(\mathbb{R}^{n}\right) .
$$

Итак, выполнены условия леммы 5 , и по утверждению этой леммы справедливо неравенство (80). Теорема доказана.

Заметим, что условие (78) означает равномерную непрерывность вектора потока $\varphi(u)$. В частности, при этом условии ограничение (3) выполнено автоматически. 
СЛЕДСТВИЕ 5 (принцип сравнения). Пусть $u_{0}, v_{0} \in L_{\mathrm{loc}}^{1}\left(\mathbb{R}^{n}\right), \quad g, h \in S$, $u_{0}(x) \leqslant v_{0}(x)$ n.в. на $\mathbb{R}^{n}, g(t, x, u) \leqslant h(t, x, u)$ п.в. на $\Pi_{T} \times \mathbb{R}$. Тогда если выполнены условия (78), (79) и $u(t, x)$ - о.ә.субр., $v(t, x)$ - о.ә.суперр. задачи (1), (2) с входныцми данными $\left(u_{0}, g\right),\left(v_{0}, h\right)$ соответственно, то $u \leqslant v$ п.в. на $\Pi_{T}$.

ДоКАЗАТЕЛЬСТВо. Предположим сначала, что дополнительно выполнено условие

$$
g(t, x, u(t, x)), h(t, x, v(t, x)) \in L_{\mathrm{loc}}^{1}\left(\Pi_{T}\right) .
$$

Тогда по теореме 7 справедливо неравенство (80), в котором $\left(u_{0}-v_{0}\right)^{+}=0$ п.в. на $\mathbb{R}^{n}, q(t, x, u, v)=0$ п.в. на $\Pi_{T}$. Здесь нужно учесть, что из непрерывности функций $g(t, x, u), h(t, x, u)$ по переменной $u \in \mathbb{R}$ и условия $g(t, x, u) \leqslant h(t, x, u)$ п.в. на $\Pi_{T} \times \mathbb{R}$ следует, что п.в. на $\Pi_{T} g(t, x, u) \leqslant h(t, x, u) \quad \forall u \in \mathbb{R}$. Поэтому $g(t, x, u(t, x)) \leqslant h(t, x, u(t, x)), g(t, x, v(t, x)) \leqslant h(t, x, v(t, x))$ п.в. на $\Pi_{T}$. По неравенству (80) $u \leqslant v$ п.в. на $\Pi_{T}$, что и нужно было доказать. Заметим, что условие (86) всегда выполнено, если $u$ и $v$ - о.э.р. задачи (1), (2). Поэтому $u^{+} \leqslant v^{-}$, где $u^{+}=J^{+}\left(u_{0}, g\right)$ - наибольшее о.э.р., а $v^{-}=J^{-}\left(v_{0}, h\right)$ - наименьшее о.э.р. задачи (1), (2) с соответствуюшими входными данными. Тогда по теореме 6 п.в. на $\Pi_{T}$ справедлива цепочка неравенств $u \leqslant u^{+} \leqslant v^{-} \leqslant v$, доказываюшая принцип сравнения в общем случае, когда условие (86) может и не выполняться.

ТЕОРема 8. При выполнении условий (78), (79) о.э.р. задачи (1), (2) единственно.

ДокАЗАТЕЛЬСТво. Если $u, v$ - о.э.р. задачи (1), (2), то по замечанию 2,c) $u, v$ являются также о.э.субр. и о.э.суперр. этой задачи. По принципу сравнения $u \leqslant v$ и $v \leqslant u$ п.в. на $\Pi_{T}$. Итак, $u=v$ п.в. на $\Pi_{T}$ и о.э.р. задачи $(1),(2)$ единственно.

Докажем, что в случае, когда одна из функций $u$ или $v$ в теореме 7 не зависит от пространственных переменных и ограничена, утверждение теоремы 7 верно без каких-либо ограничений на вектор потока $\varphi(u)$. Будем следовать схеме доказательства теоремы 2.2 из [5], [6], где разобран частный случай ограниченных суб- и суперрешений. Итак, справедлива

ТеОРема 9. Пусть $u$ - о.э.субр., $v$ - о.э.суперр. задачи (1), (2) с входными данными $\left(u_{0}, g\right),\left(v_{0}, h\right)$ соответственно такие, что для $g=g(t, x, u(t, x))$, $h=h(t, x, v(t, x)), \quad g, h \in L_{\mathrm{loc}}^{1}\left(\Pi_{T}\right), u\left(u_{0}-v_{0}\right)^{+} \in L^{1}\left(\mathbb{R}^{n}\right)$

$$
\begin{gathered}
q=q(t, x, u, v)=\chi_{u \geqslant v} \min \left\{(g(t, x, v)-h(t, x, v))^{+},\right. \\
\left.(g(t, x, u)-h(t, x, u))^{+}\right\} \in L^{1}\left(\Pi_{T}\right) .
\end{gathered}
$$

Предположим также, что одна из функиий и или $v$ не зависит от $x$ и ограничена. Тогда для п.в. $\tau \in(0, T)$ справедлива оченка (80).

ДоКАЗАТЕЛЬСТво. Ввиду замечания $2, \mathrm{~b})$ достаточно рассмотреть случай, когда функция $v$ не зависит от $x$ и ограничена: $v=v(t),|v| \leqslant M=$ const п.в. на $\Pi_{T}$. Тогда $v(t)+s$ также будет о.э.суперр. задачи $(1),(2)$ с входными данными $\left(v_{0}+s, h\right)$, $h=h(t, x, v(t))$, для любого $s \in \mathbb{R}$. По лемме 6

$$
\begin{gathered}
\frac{\partial}{\partial t}(u-v-s)^{+}+\operatorname{div}_{x}\left[\operatorname{sign}^{+}(u-v-s)(\varphi(u)-\varphi(v+s))\right] \\
\leqslant(g-h)^{+} \chi_{u-v \geqslant s} \quad \text { в } \mathscr{D}^{\prime}\left(\Pi_{T}\right) .
\end{gathered}
$$


Пусть $m>n-1$,

$$
\rho(s)= \begin{cases}0, & s \leqslant 0, \\ s^{m}, & 0<s \leqslant 1, \\ 1, & s>1,\end{cases}
$$

$\beta(s)=\rho(s / \delta)$, где $\delta \in(0,1), \eta(u)=\int_{-\infty}^{u} \beta(s) d s$. Умножим неравенство (87) на неотрицательную финитную функцию $\beta^{\prime}(s)$ и проинтегрируем по $s$. Применяя формулу интегрирования по частям, получим

$$
\eta(u-v)_{t}+\operatorname{div}_{x} \psi(u, v) \leqslant \beta(u-v)(g-h)^{+} \quad \text { в } \mathscr{D}^{\prime}\left(\Pi_{T}\right),
$$

где

$$
\psi(u, v)=\int_{0}^{u-v}(\varphi(u)-\varphi(v+s)) \beta^{\prime}(s) d s \in \mathbb{R}^{n},
$$

т.е. $\forall f=f(t, x) \in C_{0}^{\infty}\left(\Pi_{T}\right), f \geqslant 0$, имеем

$$
\int_{\Pi_{T}}\left[\eta(u-v) f_{t}+\left(\psi(u, v), \nabla_{x} f\right)+\beta(u-v)(g-h)^{+} f\right] d t d x \geqslant 0 .
$$

С учетом (3) при $s<u-v, s \in \operatorname{supp} \beta^{\prime}(s)=[0, \delta]$, верна оценка

$$
\begin{aligned}
|\varphi(u)-\varphi(v+s)| & \leqslant C_{0}(|u|+|v+s|+2) \\
& \leqslant C_{0}((u-v-s)+2|v+s|+2) \\
& \leqslant C_{0}(u-v-s)+2 C_{0}(|v|+s+1) \leqslant C_{0}(u-v-s)+C_{1},
\end{aligned}
$$

где $C_{1}=(2 M+4) C_{0}$ (напомним, что $\left.\delta<1\right)$, из которой следует, что

$$
\begin{aligned}
|\psi(u, v)| & \leqslant C_{0} \int_{0}^{u-v}(u-v-s) \beta^{\prime}(s) d s+C_{1} \int_{0}^{u-v} \beta^{\prime}(s) d s \\
& =C_{0} \eta(u-v)+C_{1} \beta(u-v) .
\end{aligned}
$$

Пусть $\lambda(\varepsilon)=\sup |\psi(u, v)| /(\eta(u-v)+\varepsilon)$, где $\varepsilon>0$. С учетом полученной выше оценки для $|\psi(u, v)|$

$$
\begin{aligned}
\lambda(\varepsilon) & \leqslant C_{0}+C_{1} \sup _{r>0} \frac{\beta(r)}{\eta(r)+\varepsilon}=C_{0}+C_{1} \sup _{r>0} \frac{\rho(r)}{\delta \int_{0}^{r} \rho(s) d s+\varepsilon} \\
& =C_{0}+C_{1} \sup _{0<r \leqslant 1}\left(\frac{\delta}{m+1} r+\varepsilon r^{-m}\right)^{-1} .
\end{aligned}
$$

Поскольку при $\varepsilon<\frac{\delta}{m(m+1)}$

$$
\sup _{r>0}\left(\frac{\delta}{m+1} r+\varepsilon r^{-m}\right)^{-1}=\left(\frac{m}{\delta}\right)^{\frac{m}{m+1}}((m+1) \varepsilon)^{-\frac{1}{m+1}}
$$

и $m>n-1$, то из (89) следует, что

$$
\limsup _{\varepsilon \rightarrow 0} \varepsilon(\lambda(\varepsilon))^{n}=0 .
$$


Заметим, что по определению величины $\lambda(\varepsilon)$ справедливы оценки компонент вектора $\psi(u, v)$ :

$$
\left|\psi_{i}(u, v)\right| \leqslant|\psi(u, v)| \leqslant \lambda(\varepsilon)(\eta(u-v)+\varepsilon), \quad i=1, \ldots, n .
$$

Тогда из (88) вытекает, что при $w=\eta(u-v)$ выполнено интегральное неравенство: $\forall f=f(t, x) \in C_{0}^{\infty}\left(\Pi_{T}\right), f \geqslant 0$,

$$
\int_{\Pi_{T}}\left\{w f_{t}+\sum_{i=1}^{n}(w+\varepsilon) \lambda(\varepsilon)\left|f_{x_{i}}\right|+\beta(u-v)(g-h)^{+} f\right\} d t d x \geqslant 0 .
$$

Далее, ввиду (81) и очевидного неравенства $\beta(s) s \leqslant(m+1) \eta(s)$ имеем

$$
\begin{gathered}
\beta(u-v)(g-h)^{+} \leqslant \beta(u-v)\left(L(u-v)^{+}+q(t, x, u, v)\right) \\
\leqslant L(m+1) \eta(u-v)+q(t, x, u, v) \\
q=q(t, x, u, v)=\chi_{u \geqslant v} \min \left\{(g(t, x, v)-h(t, x, v))^{+},(g(t, x, u)-h(t, x, u))^{+}\right\} \\
L=\max (L(g), L(h)) .
\end{gathered}
$$

Тогда из (91) вытекает, что $\forall f=f(t, x) \in C_{0}^{\infty}\left(\Pi_{T}\right), f \geqslant 0$,

$$
\int_{\Pi_{T}}\left\{w f_{t}+\sum_{i=1}^{n}(w+\varepsilon) \lambda(\varepsilon)\left|f_{x_{i}}\right|+(L(m+1) w+q) f\right\} d t d x \geqslant 0 .
$$

Ввиду (90) выполнено условие (66) леммы 5. Заметим также, что при $w_{0}=$ $\eta\left(u_{0}-v_{0}\right)$

$$
\left(w-w_{0}\right)^{+} \leqslant\left((u-v)^{+}-\left(u_{0}-v_{0}\right)^{+}\right)^{+} \leqslant\left(u-u_{0}\right)^{+}+\left(v_{0}-v\right)^{+}
$$

и, значит, выполнено условие (68). Тогда по лемме $5 w=\eta(u(\tau, x)-v(\tau, x)) \in$ $L^{1}\left(\mathbb{R}^{n}\right)$ для п.в. $\tau \in(0, T)$ и

$$
\begin{aligned}
\int_{\mathbb{R}^{n}} w(\tau, x) d x & \leqslant e^{L(m+1) \tau}\left(\int_{\mathbb{R}^{n}} w_{0}(x) d x+\int_{\Pi_{\tau}} q(t, x, u, v) d t d x\right) \\
& \leqslant e^{L(m+1) \tau}\left(\int_{\mathbb{R}^{n}}\left(u_{0}(x)-v_{0}(x)\right)^{+} d x+\int_{\Pi_{\tau}} q(t, x, u, v) d t d x\right),
\end{aligned}
$$

откуда в пределе при $\delta \rightarrow 0$ (тогда $\eta(u-v) \rightarrow(u-v)^{+}$) получим с использованием леммы $\Phi$ ату, что $(u-v)^{+} \in L^{1}\left(\Pi_{T}\right)$. Ввиду $(92)$ тогда $\beta(u-v)(g-h)^{+} \in L^{1}\left(\Pi_{T}\right)$. Из (91) по лемме 5 с $L=0, q=\beta(u-v)(g-h)^{+}$следует, что для п.в. $\tau \in(0, T)$ и всех рациональных $\delta>0$

$$
\begin{aligned}
\int_{\mathbb{R}^{n}} w(\tau, x) d x & \leqslant \int_{\mathbb{R}^{n}} w_{0}(x) d x+\int_{\Pi_{\tau}} \beta(u-v)(g-h)^{+} d t d x \\
& \leqslant \int_{\mathbb{R}^{n}}\left(u_{0}(x)-v_{0}(x)\right)^{+} d x+\int_{\Pi_{\tau}} \chi_{u \geqslant v}(g-h)^{+} d t d x,
\end{aligned}
$$

5

Серия математическая, №6 
откуда, переходя к пределу при $\delta \rightarrow 0$ и учитывая оценку (81), получим, что для п.в. $\tau \in(0, T)$

$$
\begin{aligned}
\int_{\mathbb{R}^{n}}(u(\tau, \cdot)-v(\tau, \cdot))^{+} d x \leqslant & \int_{\mathbb{R}^{n}}\left(u_{0}(x)-v_{0}(x)\right)^{+} d x \\
& +\int_{\Pi_{\tau}}\left[L(u-v)^{+}+q(t, x, u, v)\right] d t d x .
\end{aligned}
$$

Для завершения доказательства осталось применить лемму Гронуолла, из которой и вытекает неравенство (80).

На базе теоремы 9 получим ниже оценки $L^{p}$-норм о.э.р., обобщающие результаты работ [16], [17], [5], [6].

Нам понадобится следующая техническая

Лемма 7. Пусть $G(t) \in L^{1}([0, T]), \quad F(t) \in L^{\infty}([0, T]), \quad F(t) \geqslant 0, C \geqslant 0$, $p>1$ u для n.в. $\tau \in(0, T)$

$$
F(\tau) \leqslant C^{p}+p \int_{0}^{\tau} F(t)^{\frac{p-1}{p}} G(t) d t .
$$

Тогда для п.в. $\tau \in(0, T)$ выполнено неравенство

$$
F(\tau) \leqslant\left(C+\int_{0}^{\tau} G(t) d t\right)^{p}
$$

ДоКАЗАТЕЛЬСТво. Пусть $H(\tau)=p \int_{0}^{\tau} F(t)^{\frac{p-1}{p}} G(t) d t$. Тогда $H(\tau)$-абсолютно непрерывная функция на отрезке $[0, T]$ и с учетом (93)

$$
H^{\prime}(\tau)=p F(\tau)^{\frac{p-1}{p}} G(\tau) \leqslant p G(\tau)\left(C^{p}+H(\tau)\right)^{\frac{p-1}{p}}
$$

п.в. на $(0, T)$, откуда следует, что

$$
\frac{1}{p}\left(C^{p}+H(t)\right)^{\frac{1}{p}-1} H^{\prime}(t) \leqslant G(t) .
$$

Интегрируя по $t \in(0, \tau)$, получим, что $\left(C^{p}+H(\tau)\right)^{\frac{1}{p}} \leqslant C+\int_{0}^{\tau} G(t) d t$, и ввиду (93)

$$
F(\tau) \leqslant C^{p}+H(\tau) \leqslant\left(C+\int_{0}^{\tau} G(t) d t\right)^{p}
$$

что и требовалось доказать.

Teоpema 10. Пусть прu $1 \leqslant p \leqslant \infty \quad u_{0}(x) \in L^{p}\left(\mathbb{R}^{n}\right), \quad g_{0}(t, x)=g(t, x, 0) \in$ $L^{1}\left((0, T), L^{p}\left(\mathbb{R}^{n}\right)\right) ; u=u(t, x)$ - о.ә.p. задачи (1), (2). Тогда $u(\tau, \cdot) \in L^{p}\left(\mathbb{R}^{n}\right)$ для п.в. $\tau \in(0, T) u$

$$
\|u(\tau, \cdot)\|_{p} \leqslant e^{L \tau}\left(\left\|u_{0}\right\|_{p}+\int_{0}^{\tau}\left\|g_{0}(t, \cdot)\right\|_{p} d t\right), \quad L=L(g) .
$$


ДоКАЗАТЕЛЬСТВО. Пусть $k(t)$ - абсолютно непрерывная функция на отрезке $[0, T], k(t)>0$ и $\left(\left|u_{0}\right|-k(0)\right)^{+} \in L^{1}\left(\mathbb{R}^{n}\right),\left(\left|g_{0}\right|+L k-k^{\prime}\right)^{+} \in L^{1}\left(\Pi_{T}\right)$. Ясно, что функции $\pm k(t)$ являются о.э.р. задачи (1), (2) с входными данными $\pm k(0), \pm k^{\prime}(t)$. Применяя теорему 9 к о.э.р. $u, v=k(t)$ и учитывая, что по (4)

$$
\begin{aligned}
q(t, x, u, v) & \leqslant \chi_{u \geqslant k(t)}\left(g(t, x, k(t))-k^{\prime}(t)\right)^{+} \\
& \leqslant \chi_{u \geqslant k(t)}\left(|g(t, x, 0)|+L k(t)-k^{\prime}(t)\right)^{+} \in L^{1}\left(\Pi_{T}\right),
\end{aligned}
$$

получим, что для п.в. $\tau \in(0, T)$

$$
\begin{aligned}
\int_{\mathbb{R}^{n}}(u(\tau, x)-k(\tau))^{+} d x \leqslant & e^{L \tau}\left(\int_{\mathbb{R}^{n}}\left(u_{0}-k(0)\right)^{+} d x\right. \\
& \left.+\int_{\Pi_{\tau}} \chi_{u \geqslant k}\left(\left|g_{0}\right|+L k-k^{\prime}\right)^{+} d t d x\right) .
\end{aligned}
$$

Аналогично, по теореме 9 , примененной к о.э.p. $-k(t)$ и $u$ (тогда

$$
\begin{aligned}
q(t, x, u, v) & \leqslant \chi_{u \leqslant-k(t)}\left(-k^{\prime}(t)-g(t, x,-k(t))\right)^{+} \\
& \leqslant \chi_{u \leqslant-k(t)}\left(|g(t, x, 0)|+L k(t)-k^{\prime}(t)\right)^{+} \in L^{1}\left(\Pi_{T}\right)
\end{aligned}
$$

ввиду (4)), справедливо неравенство: для п.в. $\tau \in(0, T)$

$$
\begin{aligned}
\int_{\mathbb{R}^{n}}(-u(\tau, x)-k(\tau))^{+} d x \leqslant & e^{L \tau}\left(\int_{\mathbb{R}^{n}}\left(-u_{0}-k(0)\right)^{+} d x\right. \\
& \left.+\int_{\Pi_{\tau}} \chi_{u \leqslant-k}\left(\left|g_{0}\right|+L k-k^{\prime}\right)^{+} d t d x\right) .
\end{aligned}
$$

Суммируя (95) и (96), приходим к оценке

$$
\begin{aligned}
\int_{\mathbb{R}^{n}}(|u(\tau, x)|-k(\tau))^{+} d x \leqslant & e^{L \tau}\left(\int_{\mathbb{R}^{n}}\left(\left|u_{0}\right|-k(0)\right)^{+} d x\right. \\
& \left.+\int_{\Pi_{\tau}} \chi_{|u| \geqslant k}\left(\left|g_{0}\right|+L k-k^{\prime}\right)^{+} d t d x\right)
\end{aligned}
$$

для п.в. $\tau \in(0, T)$. Рассмотрим сначала случай $p=\infty$. Пусть при $\delta>0$

$$
k(t)=e^{L t}\left(\delta(1+t)+\left\|u_{0}\right\|_{\infty}+\int_{0}^{t}\left\|g_{0}(s, \cdot)\right\|_{\infty} d s\right) .
$$

Тогда $k(0)=\delta+\left\|u_{0}\right\|_{\infty}, k^{\prime}-L k \geqslant \delta+\left\|g_{0}(t, \cdot)\right\|_{\infty}$ и $\left(\left|u_{0}\right|-k(0)\right)^{+}=0$ п.в. на $\mathbb{R}^{n}$, $\left(\left|g_{0}\right|+L k-k^{\prime}\right)^{+}=0$ п.в. на $\Pi_{T}$. Из $(97)$ тогда следует, что $|u| \leqslant k$ п.в. на $\Pi_{T}$ для любого $\delta>0$ и в пределе при $\delta \rightarrow 0$ получим (94) с $p=\infty$.

Переходя к рассмотрению случая $p<\infty$, положим $k(t)=s e^{A t}$, где $s>0$, $A=L+\delta, \delta>0$. Тогда из оценки

$$
\int_{\mathbb{R}^{n}}\left(\left|u_{0}\right|-s\right)^{+} d x \leqslant \int_{\left|u_{0}\right|>s}\left|u_{0}\right| d x \leqslant s^{1-p} \int\left|u_{0}\right|^{p} d x
$$


следует, что $\left(\left|u_{0}\right|-k(0)\right)^{+}=\left(\left|u_{0}\right|-s\right)^{+} \in L^{1}\left(\mathbb{R}^{n}\right)$. Аналогично получаем, что $\left(\left|g_{0}\right|+L k-k^{\prime}\right)^{+}=\left(\left|g_{0}\right|-\delta k\right)^{+} \leqslant\left(\left|g_{0}\right|-\delta s\right)^{+} \in L^{1}\left(\Pi_{T}\right)$. Из (97) следует, что для п.в. $\tau \in(0, T)$

$$
\begin{aligned}
\int_{\mathbb{R}^{n}}\left(|u(\tau, x)|-s e^{A \tau}\right)^{+} d x \leqslant & e^{L \tau}\left(\int_{\mathbb{R}^{n}}\left(\left|u_{0}\right|-s\right)^{+} d x\right. \\
& \left.+\int_{\Pi_{\tau}} \chi_{|u| \geqslant s e^{A t}}\left(\left|g_{0}\right|-\delta s\right)^{+} d t d x\right) .
\end{aligned}
$$

Ясно, что множество полной меры $\mathscr{E}$ значений $\tau$, для которых выполнено неравенство (98), можно выбрать общим для счетного, всюду плотного множества значений параметров $s, \delta \in \mathbb{Q}$. Из непрерывной зависимости интегралов в (98) от $s, \delta$ следует, что при $\tau \in \mathscr{E}(98)$ выполнено при всех вещественных $s, \delta>0$.

Если $p=1$, то из (98) в пределе при $s \rightarrow 0$ с использованием леммы Фату вытекает, что $\forall \tau \in \mathscr{E}$

$$
\int_{\mathbb{R}^{n}}|u(\tau, x)| d x \leqslant e^{L \tau}\left(\int_{\mathbb{R}^{n}}\left|u_{0}\right| d x+\int_{\Pi_{\tau}}\left|g_{0}\right| d t d x\right)
$$

что доказывает утверждение теоремы при $p=1$. Если же $p>1$, то, разделив неравенство (98) на $e^{A \tau}$, получим оценку: $\forall \tau \in \mathscr{E}$

$$
\begin{aligned}
\int_{\mathbb{R}^{n}}(|v(\tau, x)|-s)^{+} d x & \leqslant e^{-L \delta}\left(\int_{\mathbb{R}^{n}}\left(\left|u_{0}\right|-s\right)^{+} d x+\int_{\Pi_{\tau}} \chi_{|v| \geqslant s}\left(\left|g_{0}\right|-\delta s\right)^{+} d t d x\right) \\
& \leqslant \int_{\mathbb{R}^{n}}\left(\left|u_{0}\right|-s\right)^{+} d x+\int_{\Pi_{\tau}} \chi_{|v| \geqslant s}\left(\left|g_{0}\right|-\delta s\right)^{+} d t d x
\end{aligned}
$$

где $v=u e^{-A t}$. Пусть $R>0$,

$$
E(r)= \begin{cases}\left(r^{+}\right)^{p}, & r \leqslant R \\ R^{p}+p R^{p-1}(r-R), & r>R\end{cases}
$$

Очевидно,

$$
E^{\prime \prime}(r)= \begin{cases}p(p-1)\left(r^{+}\right)^{p-2}, & r \leqslant R, \\ 0, & r>R,\end{cases}
$$

- неотрицательная финитная суммируемая функция. Умножим неравенство (99) на $E^{\prime \prime}(s-\delta)$ и проинтегрируем по переменной $s$ с использованием формулы интегрирования по частям. Получим неравенство: $\forall \tau \in \mathscr{E}$

$$
\int_{\mathbb{R}^{n}} E(|v(\tau, x)|-\delta) d x \leqslant \int_{\mathbb{R}^{n}} E\left(\left|u_{0}\right|-\delta\right)^{+} d x+\int_{\Pi_{\tau}} E^{\prime}(|v|-\delta)\left(\left|g_{0}\right|-\delta^{2}\right)^{+} d t d x .
$$

Мы учли, что $\left(\left|g_{0}\right|-\delta s\right)^{+} \leqslant\left(\left|g_{0}\right|-\delta^{2}\right)^{+}$при $s>\delta, E(s-\delta)=0$ при $s \leqslant \delta$. Заметим также, что $E\left(\left|u_{0}\right|-\delta\right) \leqslant\left|u_{0}\right|^{p} \in L^{1}\left(\mathbb{R}^{n}\right), E^{\prime}(|v|-\delta)\left(\left|g_{0}\right|-\delta^{2}\right)^{+} \leqslant E^{\prime}(R)\left(\left|g_{0}\right|-\right.$ $\left.\delta^{2}\right)^{+} \in L^{1}\left(\Pi_{T}\right)$. Из (100) тогда следует, что $E(|v|-\delta) \in L^{\infty}\left((0, T), L^{1}\left(\mathbb{R}^{n}\right)\right)$. 
Учитывая, что $E^{\prime}(r) \leqslant p(E(r))^{\frac{p-1}{p}}$ и применяя неравенство Гёльдера, выводим неравенство

$$
\begin{aligned}
\int_{\mathbb{R}^{n}} E^{\prime}(|v|-\delta)\left(\left|g_{0}\right|-\delta^{2}\right)^{+} d x & \leqslant p \int_{\mathbb{R}^{n}}(E(|v|-\delta))^{\frac{p-1}{p}}\left|g_{0}\right| d x \\
& \leqslant p\left(\int_{\mathbb{R}^{n}} E(|v|-\delta) d x\right)^{\frac{p-1}{p}}\left\|g_{0}(t, \cdot)\right\|_{p}
\end{aligned}
$$

Тогда из этого неравенства и из (100) следует, что $\forall \tau \in \mathscr{E}$

$$
H(\tau) \leqslant \int_{\mathbb{R}^{n}}\left|u_{0}\right|^{p} d x+p \int_{0}^{\tau}(H(t))^{\frac{p-1}{p}} G(t) d t
$$

где $H(t)=\int_{\mathbb{R}^{n}} E(|v(t, x)|-\delta) d x, G(t)=\left\|g_{0}(t, \cdot)\right\|_{p}$. Тогда по лемме $7 \forall \tau \in \mathscr{E}$

$$
H(\tau) \leqslant\left(\left\|u_{0}\right\|_{p}+\int_{0}^{\tau} G(t) d t\right)^{p}
$$

Переходя в этой оценке к пределу при $\delta \rightarrow 0, R \rightarrow \infty$ и используя лемму Фату, получим, что $\forall \tau \in \mathscr{E}$

$$
\int_{\mathbb{R}^{n}}|v(\tau, x)|^{p} \leqslant\left(\left\|u_{0}\right\|_{p}+\int_{0}^{\tau} G(t) d t\right)^{p}
$$

где $v=u e^{-L t}$, что эквивалентно (94). Теорема полностью доказана.

Из доказанной теоремы следует, в частности, что при $u_{0} \in L^{\infty}\left(\mathbb{R}^{n}\right), g(t, x, 0) \in$ $L^{1}\left((0, T), L^{\infty}\left(\mathbb{R}^{n}\right)\right)$ о.э.p. $u(t, x)$ принадлежит $L^{\infty}\left(\Pi_{T}\right)$ и п.в. на $\Pi_{T}$

$$
|u(t, x)| \leqslant M=\|u\|_{\infty}+\int_{0}^{T}\|g(t, \cdot, 0)\|_{\infty} d t
$$

Если $v$ - о.э.субр. задачи (1), $(2)$, то $v \leqslant u^{+} \leqslant M$ п.в. на $\Pi_{T}$, где $u^{+}=$ $J^{+}\left(u_{0}, g\right)$ - наибольшее о.э.р. Если же $v-$ о.э.суперр. задачи $(1),(2)$, то $v \geqslant u^{-} \geqslant$ $-M$ п.в. на $\Pi_{T}$, где $u^{-}=J^{-}\left(u_{0}, g\right)$ - наименьшее о.э.р. Отсюда следует, что условия теорем 7,8 в рассматриваемом случае можно уточнить: достаточно, чтобы требование (78) выполнялось при $u, v \in[-M, M]$. Заметим, что из равномерной непрерывности вектора потока $\varphi(u)$ на отрезке $[-M, M]$ следует сушествование функций $\omega_{i}(r)$ в условии $(78)$ таких, что $\omega_{i}(r) \rightarrow 0$ при $r \rightarrow 0$. Тогда, если $n=1$, условие (79) выполнено автоматически и, в частности, верна единственность о.э.р. задачи (1), (2) без каких-либо ограничений на функцию потока $\varphi(u)$. Однако уже при $n=2$ единственность ограниченного о.э.р. может нарушаться (соответствующие примеры построены в [16], [14], [15], где также показано, что достаточные условия единственности (78), (79) в определенном смысле близки к необходимым). 


\section{§ 5. Единственность о.э.р. в случае периодических входных данных}

Пусть $\xi=\left\{\xi_{1}, \ldots, \xi_{n}\right\}$ - базис в $\mathbb{R}^{n}$. Функции $u(x) \in L_{\text {loc }}^{1}\left(\mathbb{R}^{n}\right), u(t, x) \in$ $L_{\text {loc }}^{1}\left(\Pi_{T}\right)$ будут называться $\xi$-периодическими, если $u\left(x+\xi_{i}\right)=u(x)$ п.в. на $\mathbb{R}^{n}$ и, соответственно, $u\left(t, x+\xi_{i}\right)=g(t, x)$ п.в. на $\Pi_{T}$ для всех $i=1, \ldots, n$. Аналогично определяется $\xi$-периодичность функций $g(t, x, u) \in S$.

Предположим, что входные данные $\left(u_{0}(x), g(t, x, u)\right)$ задачи $(1),(2)$ являются $\xi$-периодическими функциями. Тогда верна следующая

ЛЕмма 8. Наибольшее и наименьшее о.э.р. задачи (1), (2) являются $\xi$ периодическими функчиями.

ДокАЗАТЕльСТво. Пусть $u^{+}(t, x)$ - наибольшее о.э.р. задачи $(1),(2), \xi=\xi_{i}$, $1 \leqslant i \leqslant n$. Тогда функции $u^{+}(t, x \pm \xi)-$ o.э.р. этой же задачи ввиду $\xi$-периодичности входных функций $\left(u_{0}(x), g(t, x, u)\right)$. Так как $u^{+}(t, x)$ - наибольшее о.э.р., верны неравенства $u^{+}(t, x+\xi) \leqslant u^{+}(t, x), u^{+}(t, x-\xi) \leqslant u^{+}(t, x)$ п.в. на П $T$. Применяя преобразование сдвига к последнему неравенству, запишем его в виде $u^{+}(t, x) \leqslant$ $u^{+}(t, x+\xi)$ п.в. на $\Pi_{T}$. Отсюда и из первого неравенства следует, что $u^{+}(t, x+\xi)=$ $u^{+}(t, x)$ п.в. на $\Pi_{T}, \xi=\xi_{i}, i=1, \ldots, n$. Итак, $u^{+}(t, x)-\xi$-периодическая функция. Аналогично доказьвается $\xi$-периодичность наименьшего о.э.р. $u^{-}(t, x)$.

Tеорема 11. О.ә.р. задачи (1), (2) единственно.

ДоКАЗАТЕЛЬСТВо. Применяя невырожденное линейное преобразование пространственных переменных, сведем задачу к случаю, когда $\xi_{i}=e_{i}, i=1, \ldots, n,-$ канонический базис пространства $\mathbb{R}^{n}$. Пусть $u^{+}(t, x)$ и $u^{-}(t, x)$ - наибольшее и наименьшее о.э.р. задачи (1), (2). Нужно лишь установить, что эти о.э.р. равны п.в. на $\Pi_{T}$.

Выберем функции $p(y) \in C_{0}^{\infty}\left(\mathbb{R}^{n}\right), q(t) \in C_{0}^{\infty}([0, T)), p, q \geqslant 0, \int_{\mathbb{R}^{n}} p(y) d y=1$, и положим $p_{\nu}(x)=\nu^{-n} p(x / \nu), \nu \in \mathbb{N}$. Так как функции $u^{+}, u^{-}-$o.э.р. задачи $(1)$, (2), они удовлетворяют тождеству (13). Вычитая эти тождества для о.э.p. $u^{+}, u^{-}$ с пробной функцией $h(t, x)=p_{\nu}(x) q(t)$, получим равенство

$$
\begin{aligned}
\int_{\Pi_{T}}\left[\left(u^{+}-u^{-}\right) q^{\prime}(t)+\left(g\left(t, x, u^{+}\right)-g\left(t, x, u^{-}\right)\right) q(t)\right] p_{\nu}(x) d t d x \\
+\nu^{-n-1} \int_{\Pi_{T}}\left(\varphi\left(u^{+}\right)-\varphi\left(u^{-}\right), \nabla_{y} p(x / \nu)\right) q(t) d t d x=0 .
\end{aligned}
$$

Ввиду (4) $g\left(t, x, u^{+}\right)-g\left(t, x, u^{-}\right) \leqslant L\left(u^{+}-u^{-}\right)$и из (102) следует неравенство

$$
\int_{\mathbb{R}^{n}} J_{1}(x) p_{\nu}(x) d x \geqslant-\nu^{-n-1} \int_{\mathbb{R}^{n}}\left(J(x), \nabla_{y} p(x / \nu)\right) d x,
$$

где

$$
\begin{aligned}
J_{1}(x) & =\int_{0}^{T}\left(u^{+}-u^{-}\right)\left[q^{\prime}(t)+L q(t)\right] d t, \\
J(x) & =\int_{0}^{T}\left(\varphi\left(u^{+}\right)-\varphi\left(u^{-}\right)\right) q(t) d t \in \mathbb{R}^{n} .
\end{aligned}
$$


Сделаем замену переменных, положив $y=x / \nu$. Получим соотношение

$$
\int_{\mathbb{R}^{n}} J_{1}(\nu y) p(y) d y \geqslant-\frac{1}{\nu} \int_{\mathbb{R}^{n}}\left(J(\nu y), \nabla_{y} p(y)\right) d y
$$

По лемме $8 u^{+}$и $u^{-}$являются $\xi$-периодическими функциями, откуда следует периодичность функции $J_{1}$ и вектора $J$. Ясно также, что $J_{1} \in L_{\text {loc }}^{1}\left(\mathbb{R}^{n}\right)$. Тогда, как это хорошо известно, для любого компакта $K \subset \mathbb{R}^{n}$ последовательность $J_{1}(\nu y)$ сходится слабо в $L^{1}(K)$ к постоянной $C=\int_{P} J_{1}(y) d y, P=[0,1]^{n}$ и в пределе при $\nu \rightarrow \infty$ из соотношения (103) следует, что $C \geqslant 0$, т.е. $\forall q(t) \in C_{0}^{\infty}([0, T)), q(t) \geqslant 0$, имеем

$$
\int_{[0, T) \times P}\left(u^{+}-u^{-}\right)\left(q^{\prime}(t)+L q(t)\right) d t d x \geqslant 0
$$

По непрерывности неравенство (104) верно для всех неотрицательных гладких функций $q(t)$ на $[0, T]$ таких, что $q(T)=0$. Полагая $q(t)=e^{-2 L t}-e^{-2 L T}$, получим из (104) требуемое равенство $u^{+}=u^{-}$п.в. на $\Pi_{T}$. Теорема доказана.

СлЕДСТВИЕ 6 (принщип сравнения). Пусть $v(t, x)$ - о.э.субр., $a w(t, x)-$ о.э.суперр. задачи (1), (2) с входньлми даннылми $\left(v_{0}, g\right)$ и $\left(w_{0}, h\right)$ соответственно, и предположим, ито $v_{0}(x) \leqslant w_{0}(x)$ п.в. на $\mathbb{R}^{n}, g(t, x, u) \leqslant h(t, x, u)$ n.в. на $\Pi_{T} \times \mathbb{R}$. Если по крайней мере одни из данных $\left(v_{0}, g\right)$ или $\left(w_{0}, h\right)$ являются $\xi$-периодическими функциями, то $v(t, x) \leqslant w(t, x)$ n.в. на $\Pi_{T}$.

ДокАЗАтЕльство. Пусть $v^{ \pm}(t, x)=J^{ \pm}\left(v_{0}, g\right), w^{ \pm}(t, x)=J^{ \pm}\left(w_{0}, h\right)$. Предположим, что функции $w_{0}(x), h(t, x, u) \quad \xi$-периодические. Тогда по следствию 4 и теореме 11 имеем

$$
v(t, x) \leqslant v^{+}(t, x) \leqslant w^{+}(t, x)=w^{-}(t, x) \leqslant w(t, x) \text { п.в. на } \Pi_{T} \text {. }
$$

Случай $\xi$-периодических функций $v_{0}(x), g(t, x, u)$ разбирается аналогично:

$$
v(t, x) \leqslant v^{+}(t, x)=v^{-}(t, x) \leqslant w^{-}(t, x) \leqslant w(t, x) \text { п.в. на } \Pi_{T} .
$$

В дополнение к теореме 11 заметим, что в случае $\xi$-периодических данных задачу (1), (2) можно рассматривать как задачу Коши с пространственными переменными на многообразии $T^{n}$, где $T^{n}-n$-мерный тор. Одним из следствий теоремы 11 является существование и единственность о.э.р. этой задачи, которое понимается в смысле [18] (где исследован обший случай произвольного многообразия). Отметим также, что для класса ограниченных о.э.р., когда $u_{0} \in L^{\infty}\left(\mathbb{R}^{n}\right)$, $g=g(t, x) \in L^{1}\left((0, T), L^{\infty}\left(\mathbb{R}^{n}\right)\right)$, теорема 11 доказана в [19], [20]. 


\section{Список литературы}

1. Andreianov B.P., Bénilan Ph., Kruzhkov S. N. $L^{1}$-theory of scalar conservation law with continuous flux function // J. of Functional Analysis. 2000. V. 171. P. 15-33.

2. Barthélemy L. Probléme d'obstacle pour une équation quasilinéar du premier order // Sci. Toulouse. 1988. V. 9. № 2. P. 137-159.

3. Barthélemy L., Bénilan Ph. Subsolution for abstract evolution equations // Potential Analysis. 1992. V. 1. P. 93-113.

4. Bénilan Ph. Equation d'evolution dans un space de Banach quelconque et applications: These de Doctorat d'Etat. Centre d'Orsey. Universite de Paris-Sud., 1972.

5. Бенилан $\Phi .$, Кружков С. Н. Квазилинейные уравнения первого порядка с непрерывными нелинейностями // Докл. РАН. 1994. Т. 339. № 2. С. 151-154.

6. Bénilan Ph., Kruzhkov S. N. Conservation laws with continuous flux functions // NoDEA. 1996. V. 3. P. 395-419.

7. Crandall M. G. The semigroup approach to first order quasilinear equations in several space variables // Israel J. Math. 1972. V. 12. P. 108-122.

8. Владимиров В. С. Уравнения математической физики. 5-е изд. М.: Наука, 1988.

9. Goritsky A. Yu., Panov E. Yu. Example of nonuniqueness of entropy solutions in the class of locally bounded functions // Russian Journal of Mathematical Physics. 1999. V. 6. № 4. P. 492-494.

10. Кружков C.H. Обобщенные решения задачи Коши в целом для нелинейных уравнений первого порядка // ДАН СССР. 1969. Т. 187. №1. С. 29-32.

11. Кружков С. Н. Квазилинейные уравнения первого порядка со многими независимыми переменными // Матем. сб. 1970. Т. 81. № 2. С. 228-255.

12. Кружков C.H., Хильдебранд $\Phi$. Задача Коши для квазилинейных уравнений первого порядка в случае, когда область зависимости от начальных данных бесконечна // Вестн. Моск. ун-та. 1974. № 1. С. 93-100.

13. Кружков C. Н. Андреянов П.А. К нелокальной теории задачи Коши для квазилинейных уравнений первого порядка в классе локально-суммируемых функций // ДАН CCCP. 1975. T. 220. № 1. C. 23-26.

14. Кружков C. Н., Панов Е. Ю. Консервативные квазилинейные законы первого порядка с бесконечной областью зависимости от начальных данных // ДАН СССР. 1990. Т. 314. № 1. C. $79-84$.

15. Kruzhkov S. N., Panov E. Yu. Osgood's type conditions for uniqueness of entropy solutions to Cauchy problem for quasilinear conservation laws of the first order // Annali Univ. Ferrara-Sez. 1994-1995. V. XL. P. 31-53.

16. Панов Е. Ю. Обобщенные решения задачи Коши для квазилинейных законов сохранения. Дис. ... канд. физ.-мат. наук. М.: МГУ, 1991.

17. Панов E. Ю. О мерозначных решениях задачи Коши для квазилинейного уравнения первого порядка // Изв. РАН. Сер. матем. 1996. Т. 60. № 2. С. 107-148.

18. Панов Е. Ю. О задаче Коши для квазилинейного уравнения первого порядка на многообразии // Дифф. уравнения. 1997. Т. 33. №2. С. 257-266.

19. Панов Е. Ю. К теории обобщенных энтропийных суб- и суперрешений задачи Коши для квазилинейного уравнения первого порядка // Дифф. уравнения. 2001. Т. 37. № 2 . C. $249-257$.

20. Панов E. Ю. О наибольших и наименьших обобщенных энтропийных решениях задачи Коши для квазилинейного уравнения первого порядка // Матем. сб. 2002. Т. 193. № 5 . C. $95-112$. 